\title{
Evaluation of Appreciative Inquiry Interventions
}

By

Martin Stellnberger

\author{
A thesis \\ submitted to the Victoria University of Wellington \\ in partial fulfillment of the \\ requirements of the degree of \\ Master of Management Studies
}

Victoria University of Wellington

2010 


\section{Acknowledgements}

Completing a thesis needs more than just one person. It is the contribution of many people that makes such a work possible. Thus, I would like to acknowledge all those who provided their time and effort throughout this process. I am deeply appreciative for the guiding and motivating words from my supervisor, Urs Daellenbach. Your supervision and advice was of great value in making this thesis sound.

Thanks also to the AI consultants, practitioners and contact persons within organizations for their professional support in arranging the interviews. Especially, I would like to thank all the interviewees for providing their time and valuable feedback on using Appreciative Inquiry.

To my parents Anna and Hermann Stellnberger, thank you for giving me the feeling of being still connected to home while living abroad.

A final thank goes to my university colleagues and friends for the welcome distractions from working on this thesis. If it was for a short coffee break or 'extended' lunch, it was always nice to talk about the important things in life. Markus, Kathi and Andrew, thank you. 


\begin{abstract}
As a relatively new tool for organization development, action research, training and team building, Appreciative Inquiry (AI) has gained popularity worldwide (Messerschmidt, 2008). Whilst much research has addressed the approach and its philosophy, AI still remains an approach with little self-reflection or critique (Grant \& Humphries, 2006). It has been suggested that evaluation of past AI interventions is needed to inform managerial action in conducting future interventions and contribute to the literature on AI (Dunlap, 2008).
\end{abstract}

In this thesis, three large-scale AI interventions (between 130 and 320 participants in the summit) were evaluated. Three organizations operating in New Zealand and Australia agreed to participate in this research. Across these three organizations, data were collected through 23 semi-structured interviews of employees who were involved in the AI summit. Evaluation of these interventions was carried out by comparing the findings of the interviews with the intended goals and outcomes for each organization in initially performing an AI summit. In addition, the findings were contrasted with the existing literature on AI and recommendations for future implementations are made.

This research shows that the interviewees across the three organizations consistently reflected very positively on the AI summit. They commonly used words like 'fun', 'great', 'amazing', 'exciting', 'energizing', 'motivating' and 'making you feel special' to describe the event. Particularly, the involvement of a diverse group of people (co-workers from different management levels, customers, suppliers, external partners, etc.) in the summit was highly appreciated by the interviewees.

While the literature puts a great emphasis on the AI summit and the planning of the event, the findings indicate that the things that happen post the summit are at least as important as the actual summit itself. Two out of three organizations appear to have underestimated the importance of what should happen after the event and ultimately failed in integrating AI into their organizational processes or capturing significant benefits following the AI summit. In contrast, the third organization put a strong emphasis on planning actions that followed the summit and consequently managed to make AI part of their way of operating. 


\section{Table of Contents}

Acknowledgements ..............................................................................................

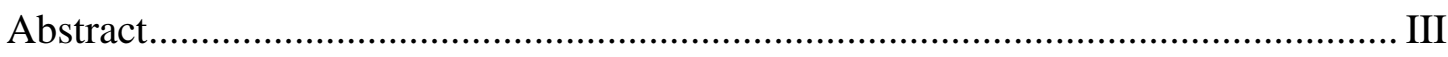

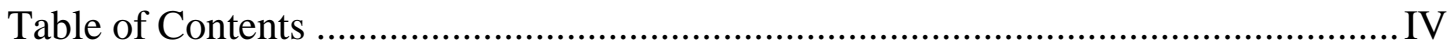

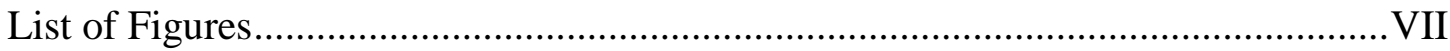

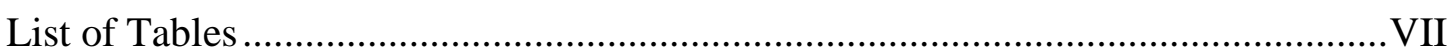

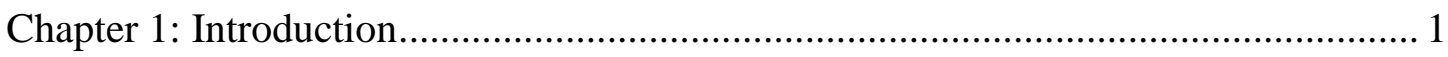

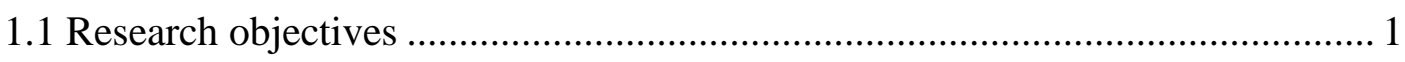

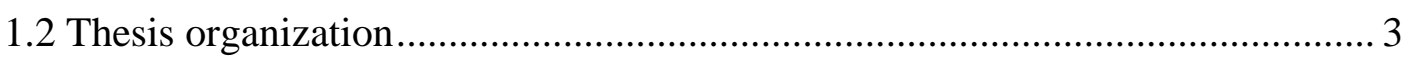

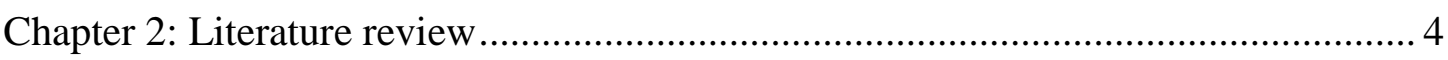

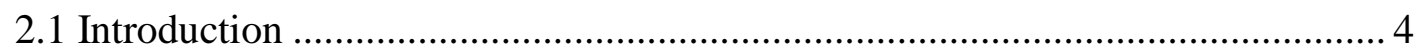

2.2 Short history of AI .................................................................................. 4

$2.3 \mathrm{AI}-$ What it is, how it works and its philosophy ………………………….... 5

2.3.1 AI - A brief introduction ......................................................................... 5

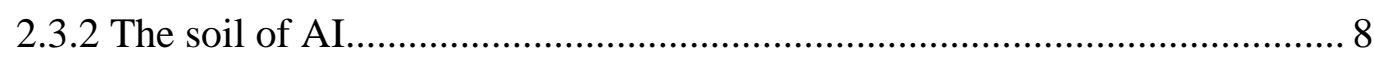

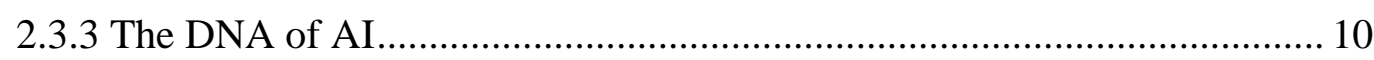

2.3.4 The practice and application of AI …………………............................ 12

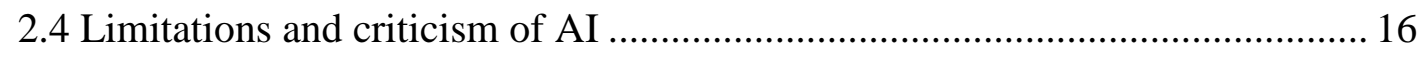

2.5 Evaluation of Appreciative Inquiry interventions ............................................ 17

2.5.1 Objectives of evaluation ........................................................................ 18

2.5.2 A 'traditional' approach to evaluation......................................................... 18

2.5.3 Existing approaches in evaluating AI...................................................... 19

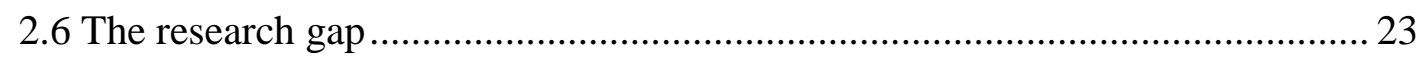

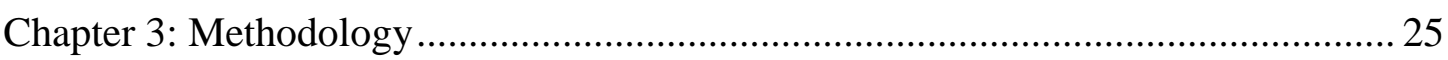

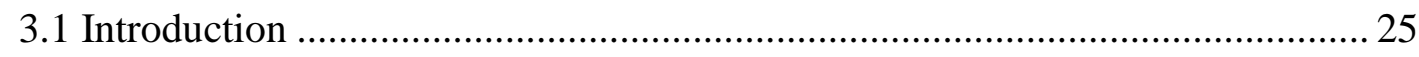

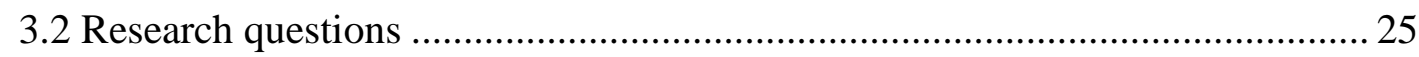

3.3 Method for data collection............................................................................... 26

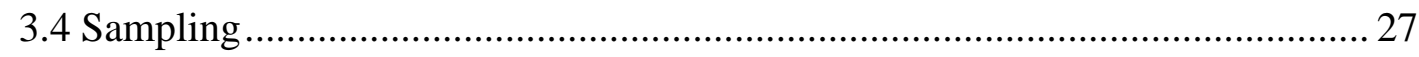

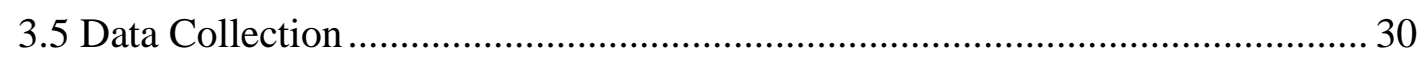

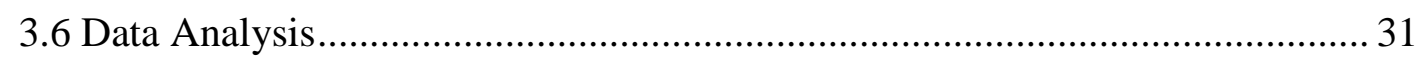

3.7 Ethical considerations and aspects of confidentiality ........................................ 33

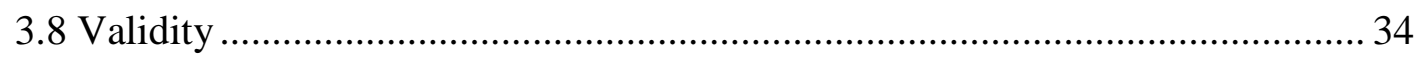

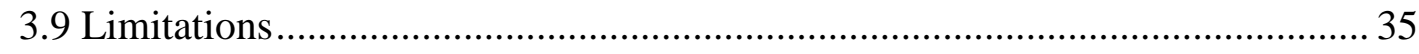




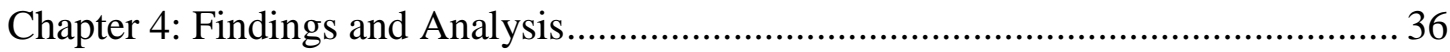

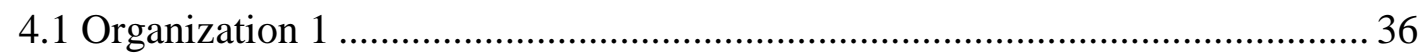

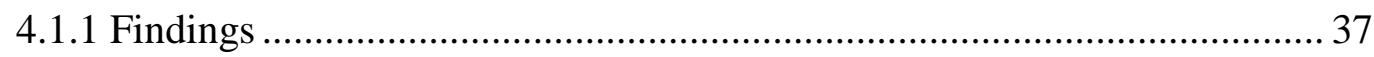

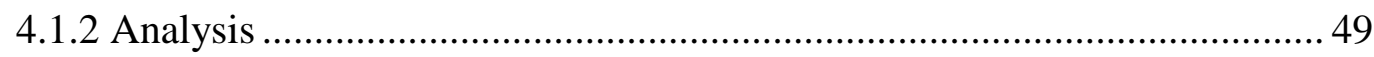

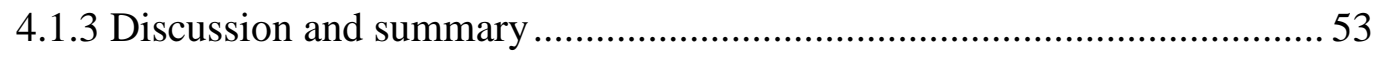

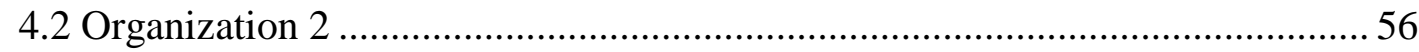

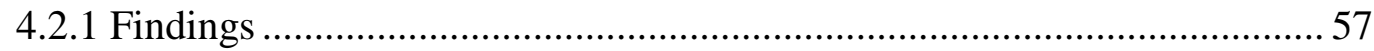

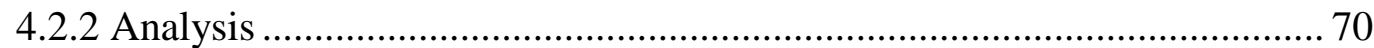

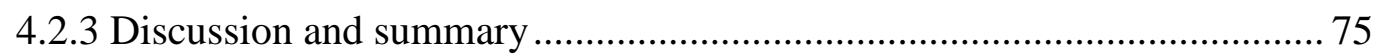

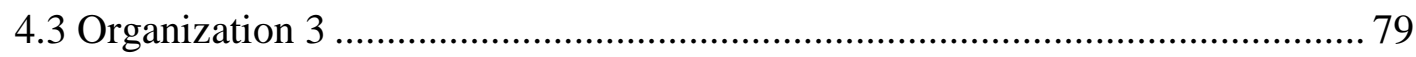

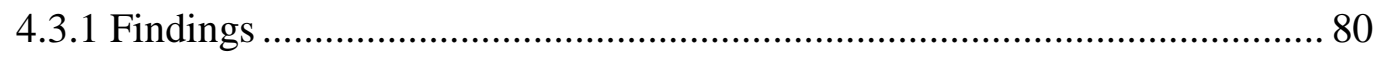

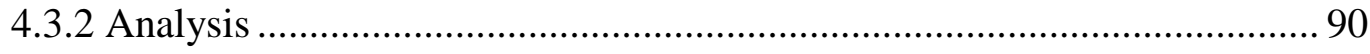

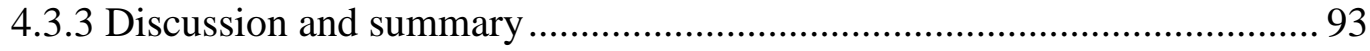

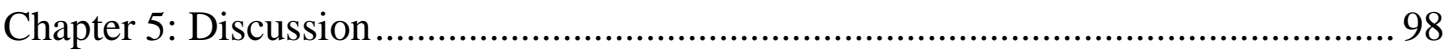

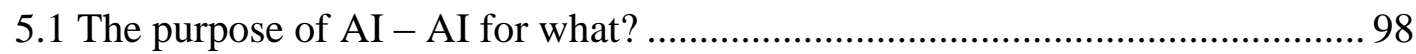

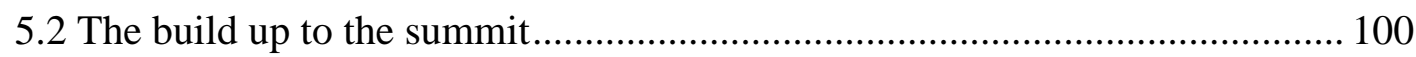

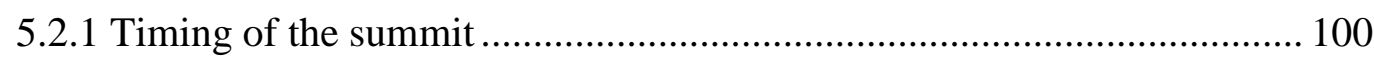

5.2.2 Preparation for the actual event ........................................................ 102

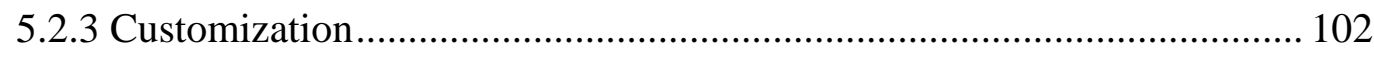

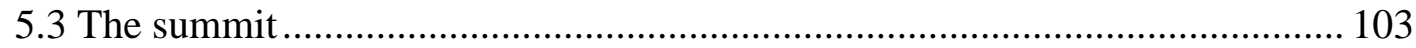

5.3.1 Appreciation of diversity among participants ........................................ 103

5.3.2 Summit results in excitement, hopes and wishes for the future .............. 104

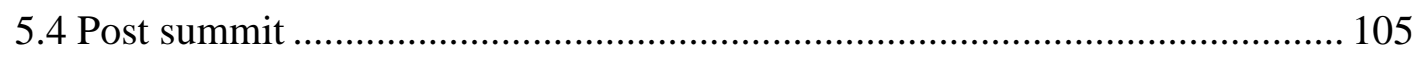

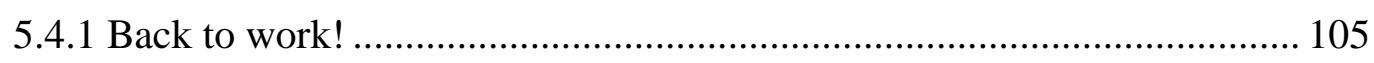

5.4.2 The case of misperceived expectations ................................................ 106

5.4.3 Provocative propositions and the follow up projects ............................. 107

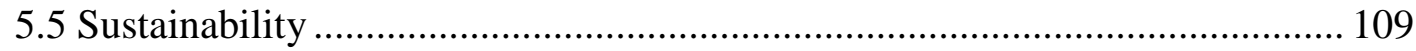

5.5.1 Involvement of the whole organization/community.............................. 110

5.5.2 Focus on achieving long-term results vs. focus on projects.................... 110

5.5.3 An argument for follow-up AI engagement meetings............................ 111

5.6 Consistency across different stakeholders of the organization....................... 112

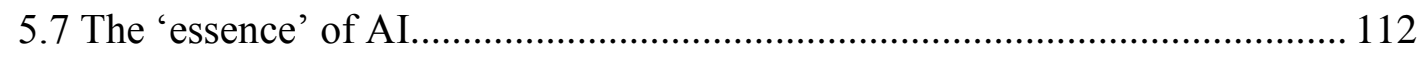

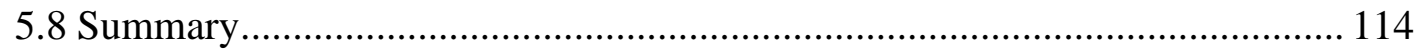

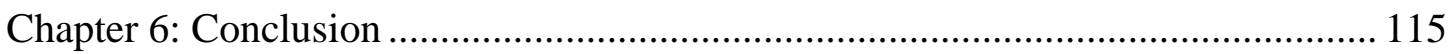




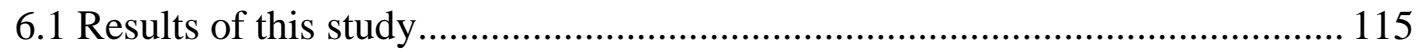

6.1.1 Ensure diversity of participants at the summit ....................................... 115

6.1.2 The summit is just the beginning........................................................... 116

6.1.3 Feed AI into organizational processes rather than projects....................... 117

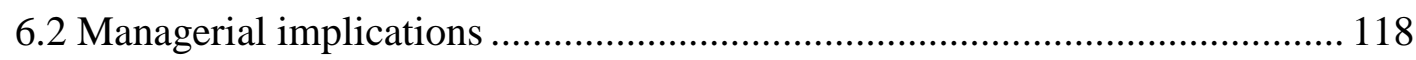

6.3 Reflection and implications for further research ………………………….... 122

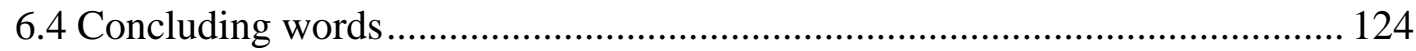

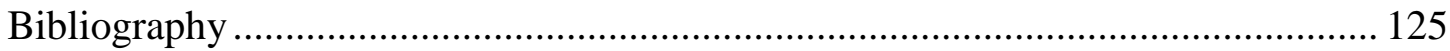

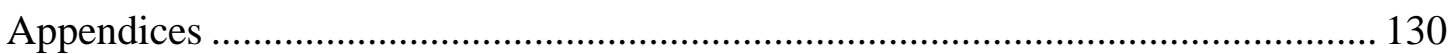

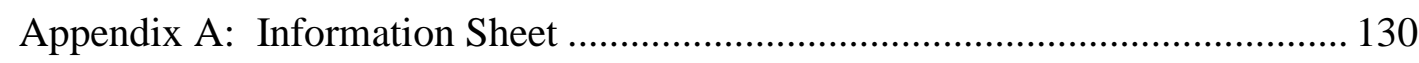

Appendix B: Research Agreement .................................................................. 131

Appendix C: Interview Schedule...................................................................... 132 


\section{List of Figures}

Figure 1: Problem solving and AI compared .......................................................... 7

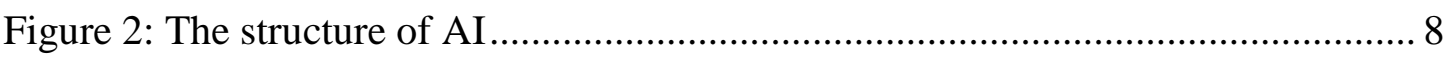

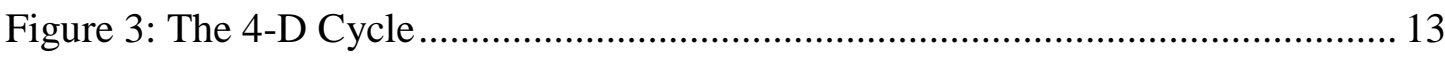

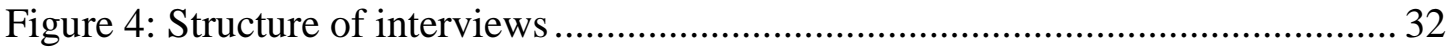

\section{List of Tables}

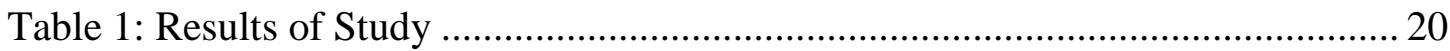

Table 2: Details on the organizations involved in this research ................................ 30

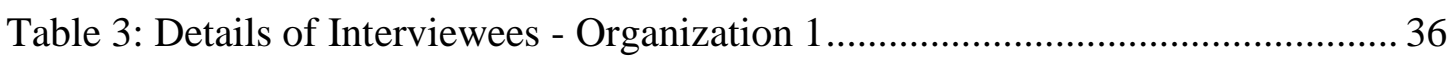

Table 4: Details of Interviewees - Organization 2 .................................................. 56

Table 5: Details of Interviewees - Organization 3 ................................................... 79

Table 6: Goals and Outcomes from the AI interventions......................................... 99 


\section{Chapter 1: Introduction}

My interest in conducting this research has its origin in my previous studies. As part of my two-year masters program, I had to conduct a research project in the first year as preparation for the second year master thesis. In searching for interesting topics, a $\mathrm{PhD}$ student introduced me to the topic of Appreciative Inquiry (AI) and provided some reading material on it. This led to me gaining a growing interest in AI, which resulted in choosing this topic for my first year research project. In this project I interviewed five AI consultants and practitioners about their views, perceptions and practical experience in using AI. While these interviewees described the benefits of using AI, its strengths, its usefulness, etc. they also recognized its shortcomings. Several interviewees pointed out that the AI methodology, as presented in the literature, does not present substantial evidence concerning its own performance. One consultant argued, “demonstrating and making AI's value tangible is actually critical in terms of getting more traction and more buy-in for utilizing the principles and concepts of AI."

Informed by the first year research project, and being aware of one of AI's perceived 'shortcomings' from a New Zealand practitioner's perspective, a review of the literature confirmed the need for more research evaluating AI interventions. Grant \& Humphries state, "despite increased applications and scholarship, appreciative inquiry remains an action research process with little self-reflection or critique" (2006, p. 402). Considering the claim made by AI practitioners and the existence of only few published AI evaluation studies (Bushe \& Kassam, 2005; Grant \& Humphries, 2006; Messerschmidt, 2008; Neumann, 2009; van der Haar \& Hosking, 2004), this research is motivated to address this gap and contribute to the literature on AI evaluation studies.

\subsection{Research objectives}

This thesis aims to contribute to the academic literature on AI as well as providing practical implications of evaluating AI interventions. This will be done by achieving the following three research objectives. 
The first objective addresses the claim made by the consultants and practitioners outlined above. Thus, this research aims to make the value of using AI in organizational settings visible, in order to get more traction and buy-in. One consultant made the argument that "until you can actually show what AI is capable of and what it can do for an organization, decision makers will hesitate in spending money on this." At this point it needs to be noted that, the objective is not to 'find' arguments on how to sell AI to organizations, this research aims to collect and analyze evidence on the performance and sustainability of AI, on which basis decision-makers can decide whether AI represents a suitable approach for use in their organization.

The academic literature on AI appears to be fairly quiet in reflecting on AI's weaknesses and deficiencies (Messerschmidt, 2008) as well as tending to point out 'just' the things that work (Bushe, 2007). This is likely due to AI's exclusive focus on positives. Thus, the second objective of this research aims to address this gap by identifying the shortcomings, difficulties etc. in applying AI in organizational settings. Building on this critique for 'self-reflection' and feedback, the research intends to put forward recommendations on how to enhance future AI interventions.

The third objective of this research is to develop and apply an approach on how AI interventions can be evaluated. Due to the philosophical assumptions AI is based upon, discussion on how evaluation needs to take these assumptions into consideration exists (van der Haar \& Hosking, 2004). This research incorporates this literature and puts forward a possible approach on how the usefulness of AI interventions can be 'measured'. Further on, this approach will be used to evaluate three AI interventions.

In this research, evaluation is performed to show what AI is capable of, to illustrate how useful it can be, and to gain insights into how AI initiatives could be improved. Considering the significant investments that are needed for applying AI in organizational settings, evaluation appears to be of critical importance in providing feedback and further establishing its legitimacy. 


\subsection{Thesis organization}

In order to achieve the outlined research objectives, this thesis is organized into the following six chapters.

The next chapter provides a review of the literature on AI. Further, relevant AI evaluation studies and the difficulties that arise in conducting evaluation are presented. The literature review concludes in outlining the gap this study intends to fill.

Chapter three contains the methodology describing the approach that is used in evaluating AI interventions. In this research, large-scale AI interventions from three separate organizations are evaluated. For this research a large-scale AI intervention is considered as involving a company wide summit with more than 100 participants. Data were collected through 23 semi-structured interviews across the three organizations (all interviewees participated in their organization's summit). Evaluation is carried out through comparing the interviewee's views, perceptions and opinions of AI, with the organization's goals and objectives in performing the intervention.

Chapter four presents the information on which basis the three AI interventions can be evaluated. For each intervention, evaluation is structured into three sections. The first section provides some background information on the organizations and the findings of the interviews. Section two analyses and condenses the findings further, while section three compares these findings with the organization's goals and objectives of doing an $\mathrm{AI}$ intervention.

Chapter five identifies and highlights several aspects of AI interventions occurring within and across the three cases. Furthermore, this chapter provides a comprehensive discussion of AI's strengths as well as weaknesses that could be identified across the cases. On this basis recommendations and suggestions for future AI interventions are made.

The last chapter summarizes the key findings of this research, puts forwards recommendations and suggestions for future interventions and provides implications for future evaluation studies. 


\section{Chapter 2: Literature review}

\subsection{Introduction}

In the previous chapter, the objectives of this research were outlined. In order to achieve these objectives, the theoretical background of AI needs to be examined first. In discussing the challenge evaluation of AI interventions faces, it is important to understand the nature and origin of this philosophy. Thus, a short review of the history starting with the doctoral work of Cooperrider (1986) is given. Following this, a detailed description of $\mathrm{AI}$ including its underlying principles and its application provides a comprehensive summary on which basis the subsequent literature on evaluating AI interventions can build on. Subsequently, this chapter reviews published approaches to evaluating AI starting with the objective of evaluation. The literature review concludes in outlining the research gap.

\subsection{Short history of AI}

While credit for popularizing AI as an organizational transformational tool is given to David Cooperrider and his colleagues, a number of other studies also indicate a positive and "appreciative" nature and either predate or were parallel to AI's development (Messerschmidt, 2008). Approaches like Asset-Based Community Development and Positive Deviance are two examples of processes in the Organizational Development literature that also reflect AI's focus on strengths and possibilities (see Buscell, 2005; Cunningham \& Mathie, 2002; Greene \& Caracelli, 1997). However, it was Cooperrider \& Srivasta (1987) who published the first article on AI, offering a set of underlying philosophical assumptions that this framework builds on. The impulse to publish their article was given in the doctoral work of Cooperrider (1986) in which he used the positive history of an organization as a source for discovering possibilities for a better future (Cooperrider, Whitney \& Stavros, 2008).

While the theoretical foundation was laid in 1987, only a handful articles were published (Jones, 1998; Whitney, 1998; Whitney \& Cooperrider, 1998; Whitney \& Schau, 1998) until AI experienced its renaissance in 1999 when Cooperrider \& Whitney (1999) published the first book on how to do AI. Since then, the number of 
published articles, doctoral dissertations and Masters theses has grown exponentially. Similar growth has occurred in other audiences of practitioners and 'Believers' (term used in Messerschmidt, 2008, p. 457).

In the recent AI Handbook (Cooperrider et al., 2008), the authors point out that AI has been successfully used in a variety of situations (e.g. strategic planning, team development, organizational culture change, meetings, new product development to accelerate organizational learning and transformation). Cooperrider et al. claim "[AI] can be used in any situation where leaders and organizational members are committed to building positive, life-centered organizations" (2008, p. XXIX).

In reviewing the literature, an early observation is that the literature on AI itself is presented in a very positive manner providing little critique. In order provide an objective review, addressing the benefits as well as the shortcomings and risks associated with AI, this chapter is organized as follows. The first section reviews AI from a positive perspective and summarizes what advocates claim AI is and how it is supposed to work. Limitations, criticism and concerns in regards to AI are covered afterwards providing the reader with a fuller and more critical perspective.

\subsection{AI - What it is, how it works and its philosophy}

In the following sections the idea and process of thought behind AI are given. In order to provide a comprehensive perspective, the paradigm AI belongs to, its methodology and method will be addressed separately.

\subsubsection{AI - A brief introduction}

To explain the term AI, Cooperrider et al. define appreciate and inquire as follows:

Ap-pre'ci-ate, v., 1. to value; recognize the best in people or the world around us; affirm past and present strengths, success, and potentials; to perceive those things that give life (health, vitality, excellence) to living systems. 2. To increase in value, e.g., the economy has appreciated in value. Synonyms: value, prize, esteem and honor.

In-quire', v., 1. to explore and discover. 2. To ask questions; to be open to seeing new potentials and possibilities. Synonyms: discover, search, systematically explore, and study.

(Cooperrider et al., 2008, p. 1) 
In the AI Handbook Cooperrider et al. (2008) describe AI as a philosophy incorporating an approach for engaging people to produce effective positive change. A practitioner definition from the handbook is as follows:

\begin{abstract}
Appreciative Inquiry is the cooperative co-evolutionary search for the best in people, their organizations, and the world around them. It involves the discovery of what gives "life" to a living system when it is most effective, alive, and constructively capable in economic, ecological, and human terms. AI involves the art and practice of asking questions that strengthen a system's capacity to apprehend, anticipate, and heighten positive potential. The inquiry is mobilized through the crafting of the 'unconditional positive question', often involving hundreds of thousands of people. AI interventions focus on the speed of imagination and innovation instead of the negative, critical, and spiralling diagnoses commonly used in organizations. The discovery, dream, design, and destiny model links the energy of the positive core to changes never thought possible.
\end{abstract}

(Cooperrider et al., 2008, p. 3)

AI is based on the assumption that something 'good' already exists in every organization, which can be discovered, used and most importantly exploited. This means that organizations should shift their focus to their strengths in order to get more of what works best, rather than eliminating what does not work (Faure, 2006; Ncube \& Wasburn, 2008). The resource-based-view (RBV) from the strategic management field presents a similar thought and motivates an organization to exploit its own valuable, rare and costly to imitate resources rather than imitating resources of a competitor. Why should an organization shift its primary focus and resources to something where it can be only average or as good as its competitors? There is little reason to do so. Consequently, the RBV argues that a focus on exploiting an organization's unique strengths and capabilities raises its chances to gain competitive advantages (Barney, 2002). As management guru Peter Drucker once stated, "the task of leadership is to create an alignment of strengths in ways that make the system's weaknesses irrelevant" (Peter Drucker cited in Salopek, 2006, p. 18).

Such initiatives represent a shift away from the problem-solving approach to the positive thinking approach. The following exhibit illustrates the difference between these two approaches. 
Figure 1: Problem solving and AI compared

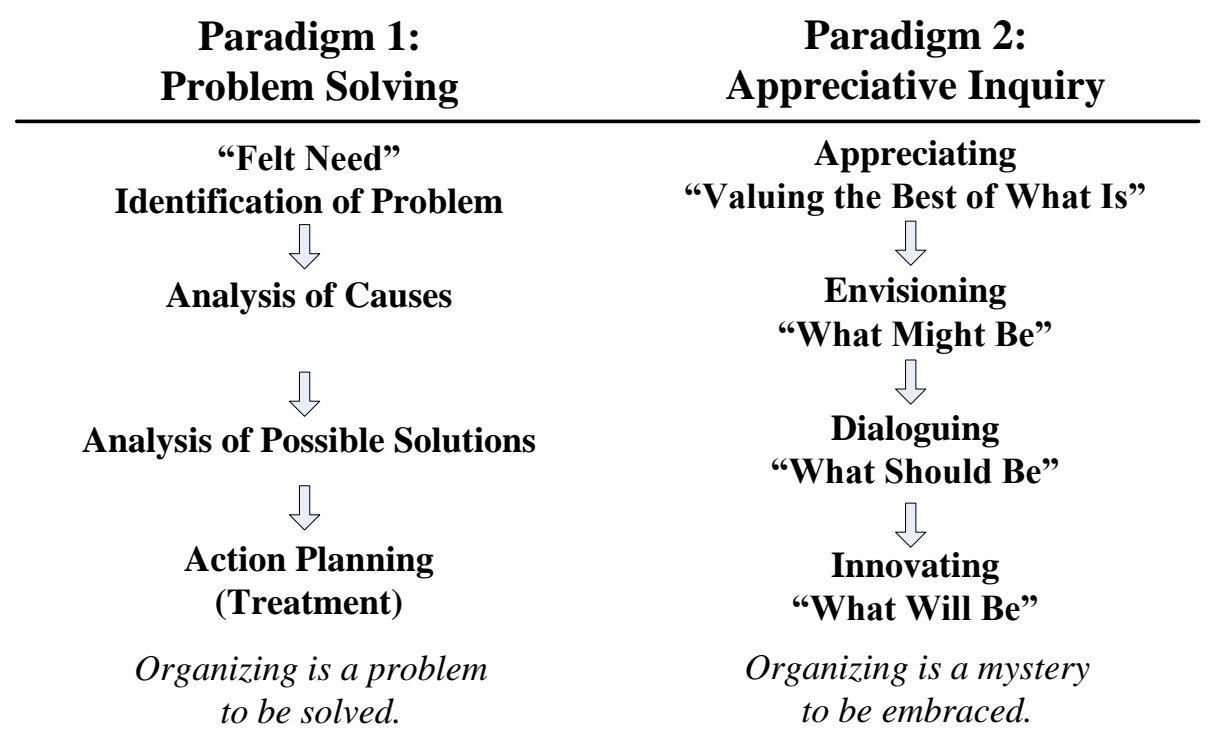

Source: Adapted from Cooperrider et al. (2008, p. 16)

Cooperrider et al. (2008) argue that no organization was created as a 'problem' and therefore organizations do not represent a problem to be solved. In fact, organizations are created to provide solutions for people, environment, etc. AI advocates claim that the problem-solving approach is limiting and negative in assuming something must be wrong which has to be repaired (Messerschmidt, 2008). As Messerschmidt states, "the AI approach seeks to 'flip' problems into their 'positive opposites' by focusing attention on the exceptions to the problems, then search for and build upon the 'root causes' of those exceptional successes" (2008, p. 455). The argument is that the traditional problem-solving approach "limits the opportunities for organizations to be successful because it reinforces existing beliefs instead of addressing the possibilities for the creation of new beliefs" (Egan \& Lancaster, 2005, p. 36).

At this stage, AI may appear to the reader to be a kind of believing game ignoring current organizational problems. To tackle this view, AI advocates might claim that AI does not ignore problems or other important issues intentionally, arguing that problems are addressed from a different (positive) angle. British Airways is a commonly used example to illustrate that point (see Cooperrider \& Whitney, 2005).

In an early stage of using AI at British Airways, employees addressed the problem of having delayed luggage at the destination airports. Through intervention of the consultants, they came up with the overachieving objective to aim for an exceptional 
customer arriving experience. The argument is that this new goal does not solve the problem itself but shifts the problem focus towards an overachieving objective to which an organization can live up to - 'having satisfied \& happy customers when they leave the airport' (Cooperrider \& Whitney, 2005).

In discussing the whole philosophy of AI and its application, the structure outlined in Watkins \& Mohr (2001) will be borrowed as a guide for reviewing AI in a comprehensive and complete manner.

Figure 2: The structure of AI

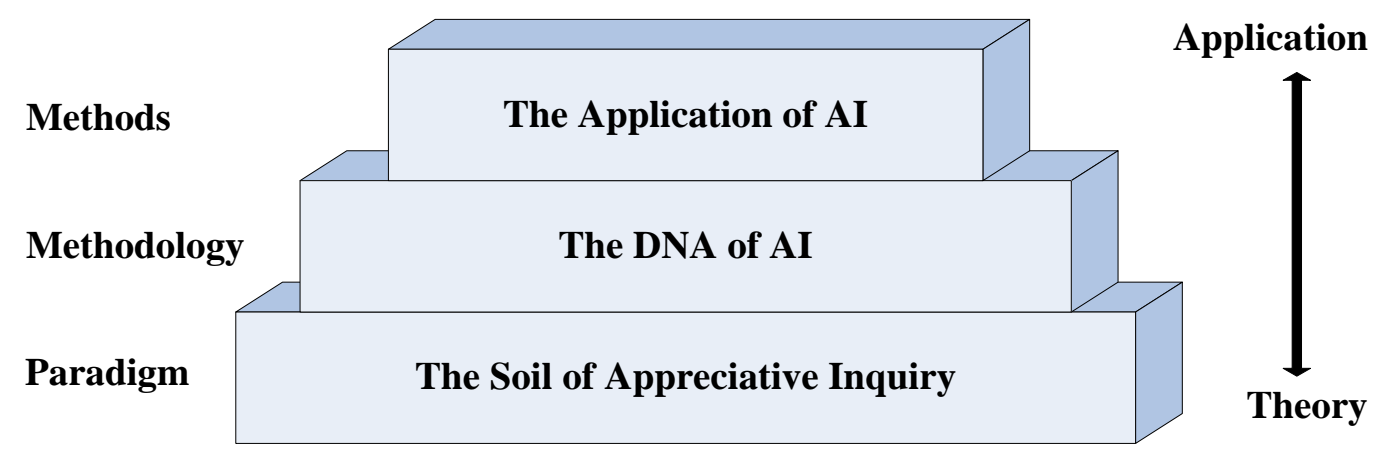

Source: Adapted from Watkins \& Mohr (2001, p. 37)

In this structure, the philosophy of $\mathrm{AI}$ is addressed at three different levels starting from its theory upwards to its application. The Soil of Appreciative Inquiry addresses the theoretical base and its philosophical assumptions about the creation of knowledge. The DNA of AI covers AI's methodology through the five core and the four guiding principles of AI. The Application of AI is presented last. One method in applying the theory of AI in an organizational area is the AI-Summit. In the following, all three levels are discussed separately starting with the soil of AI.

\subsubsection{The soil of AI}

The philosophy of AI is based on and grounded in the social constructionism paradigm (Dinesen, 2009; Faure, 2006; Watkins \& Mohr, 2001). Gergen, whose work on social constructionism had a strong impact on AI, puts it as follows:

The basic idea in social constructionism is quite simple but also quite deep. Everything that we perceive as real is socially constructed. Or said more dramatically, nothing is real before people agree that it is. 
In accepting this statement we also have to acknowledge that there are multiple realities instead of just one, which can be modified through dialogue and interaction (Faure, 2006). This means that "creating meaning emerges in relationships and not in the self, which is the more traditional viewpoint. Everything is created in relation to others" (Dinesen, 2009, p. 51). It is important to note that individuals can alter their perception of reality at any given moment and find consensus with others.

The second assumption Gergen notes is that "meaning and action are entwined. As we generate meaning together we create the future" (K. Gergen, cited in Watkins \& Mohr, 2001, p. 26). To put it differently, the present meanings and beliefs individuals hold have an impact on (or even create) future reality, i.e., what individuals think becomes reality. Three examples will be presented next to illustrate this argument where perceptions, impressions and beliefs that individuals hold presently had an impact on future outcomes.

The Placebo Effect is probably one of the best-known examples showing the impact of the mind on the body (see Cooperrider et al., 2008, p. 10ff; Watkins \& Mohr, 2001, p. 29ff). Studies show that somewhere between one-third to two-thirds of all patients show significantly improved physiological and emotional improvements in symptoms by believing they are receiving effective treatment.

The Pygmalion studies tested the impact of a teacher's perception about student performances on the actual long-term performance of students (see Cooperrider et al., 2008, p. 10ff; Watkins \& Mohr, 2001, p. 29ff). Teachers were informed that randomly chosen students are more intelligent, perform well and behave in the classroom while the others are quite the opposite. The teachers believed this information to be true and soon the categorization of these randomly chosen students turned out to become reality, i.e., the students perceived to be better performed well and the students perceived to be poor performed badly. The studies discovered that the teachers interacted with the two groups differently in terms body language and support. In short, the image teachers held about students became reality due to their differing subconscious interactions with them.

A positive correlation between positive images of ourselves and its impact on our performance and health has been found especially in the sports discipline (see 
Cooperrider et al., 2008, p. 10ff; Watkins \& Mohr, 2001, p. 29ff). The argument presented is that by just "planting" positive images of strengths, success etc. in the minds of athletes their performance will improve. The perception that elimination of failures and negative self-monitoring will enhance performance was proven wrong, when exactly the opposite turns out to be true. Further details and more examples are given in Watkins \& Mohr (2001, p. 29ff) and Cooperrider, et al. (2008, p. 10ff).

To sum up, the two assumptions presented above are:

1. Truth and meaning emerge in dialogues and relationships and not in themselves.

2. Meanings and beliefs individuals hold have an impact on (or even create) future reality.

These two assumptions represent the key foundations that AI is based on. AI advocates might claim that if these assumptions are not accepted, then the whole philosophy of AI might be difficult to agree with (see Cooperrider et al., 2008; Watkins \& Mohr, 2001). The implications for organizations and the links from these two statements to AI are presented next.

\subsubsection{The DNA of AI}

The five core principles and the four guiding principles represent the DNA of AI. Cooperrider and his colleagues purposefully avoided creating an explicit outlined method on how to do AI. The reason for doing so is that Cooperrider and his colleagues wanted to open up discussion about AI and not shut it down by providing a recipe everyone has to follow (Salopek, 2006). In the following, the guidelines for doing the inquiry appreciatively are outlined.

\section{The five core principles}

The five core principles presented are used to bridge the philosophy of AI with its application. Cooperrider et al. (2008) highlight the necessity to fully understand these principles in order to grasp its theory and its implications in applying AI.

The (1) constructionist principle states that reality is constructed during social interactions of people. Emphasis is placed on language and dialogues for establishing 
meaning, order and identity (Dunlap, 2008; Whitney, 1998). The (2) principle of simultaneity presents the view that inquiry into something and change are inseparable, i.e., the inquiry is already the intervention. Just by asking questions and engaging people in conversations, participants already alter the way they think and act (Dunlap, 2008). The (3) poetic principle states that human organizations are like an open book changing its story continuously, existing to be read and reread, interpreted and reinterpreted. Just as a book takes on meaning through the act of reading it, the organization does so too in sharing and communicating its stories (Dunlap, 2008; Whitney, 1998). The (4) anticipatory principle indicates that people's images about the present and future organization guide and influence current behavior of staff and consequently the organization's future. As Dunlap states, it is the image of the future that "will guide us in determining how we will achieve the future" $(2008$, p. 26). The (5) positive principle builds on the assumption that organizations move in the direction they are inquiring. This principle claims that questions asking and inquiring about positives and strengths of an organization will create momentum for change (Cooperrider \& Whitney, 2005). Even though these five principles are relatively abstract, they represent and serve as the foundation for understanding how AI is supposed to work and AI proponents argue that they should be considered in all AI interventions (Dunlap, 2008).

As a result of ongoing research, further principles have been identified. While the five core principles presented above are broadly accepted in the literature and form part of the latest version of the AI handbook, the further principles as presented in Dunlap (2008) or Whitney \& Trosten-Bloom (2003) are not acknowledged, indicating that debate about their legitimacy may still be going on.

A critical note: The five core principles presented above as well as the four guiding principles outlined next are reviewed on what AI-advocates claim they are and mean. The literature provides little information tackling and reviewing these principles individually. Instead some authors went over and 'acknowledged' these principles but tackled AI's philosophy as a whole (see Bushe \& Kassam, 2005; Messerschmidt, 2008). The findings of these authors are presented later on. 


\section{The four guiding principles}

The four guiding principles first mentioned in Cooperrider \& Srivastva (1987) state that

1. The inquiry begins with appreciation.

2. The inquiry is applicable.

3. The inquiry is provocative.

4. The inquiry is collaborative.

The assumption of the first principle is that every organization has something that 'works' to some degree that can be used as inspiration to inquire further into the positive and build on these moments of success. The second principle claims that the outcomes of an inquiry should seem feasible to the organization. The third principle states that the inquiry should create compelling outcomes that motivate participants to take action. The last principle states that the stakeholders of an organization should be part of the intervention (Cooperrider \& Srivastva, 1987; Faure, 2006; Messerschmidt, 2008). A Chinese proverb echoes these points "Tell me and I forget. Teach me and I may remember. Involve me and I will understand" (in Faure, 2006, p. 25).

As mentioned earlier, the five core principles and the four guiding principles describe the DNA of AI and represent its methodology. The 'founders' of AI provided these principles to build a methodology around AI but also highlight the importance to remember and consider them in every intervention (Cooperrider \& Whitney, 1999, 2005).

\subsubsection{The practice and application of AI}

So far, the philosophical assumptions of AI and its methodology were presented. In moving from theory to application, the method of AI comes next.

\section{The AI-Summit}

One of the most commonly used intervention models of AI is the AI-Summit (Faure, 2006). The summit is usually a two-day, large-scale meeting process around an affirmative topic. The purpose of such an intervention is to generate organizational 
movement towards the affirmative topic. The affirmative topic is a positively formulated phrase developed at an early stage of the intervention and represents the organization's focus of the intervention. The level of focus implied through the affirmative topic can vary significantly. Consequently, summits can be held on specific topics as well as broad topics relevant to the whole organization (Cooperrider et al., 2008).

\section{The 4-D cycle}

The model on how to undertake an AI-Summit is usually expressed through the 4-D cycle in which the whole process of an intervention is split up into four phases as shown in the following figure.

Figure 3: The 4-D Cycle

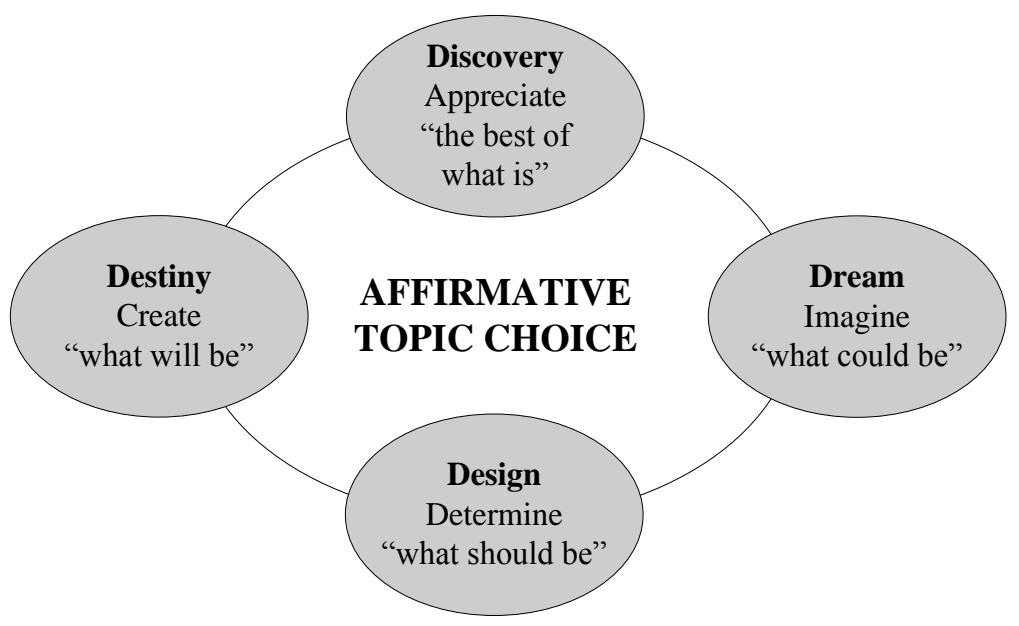

Source: Ludema, Whitney, Mohr \& Griffin (2003, p. 10)

The goal of the discovery phase (first phase) is to identify, learn and appreciate the best of what exists within an organization by focusing on times of organizational peak experiences and past success (Berrisford, 2005). Appreciative interviews are the main form of engagement in gathering data in this phase. Participants (usually pair wise) ask another member positive questions (derived from the affirmative topic) about the past encouraging them to share personal stories of excellence. Once these stories are collected, the 'best' are communicated and shared with all participants (Whitney, 1998). In building on AI's philosophy, good stories to share and retell are those, in which participants get most excited and feel engaged in them. 
In reviewing the literature, the discovery phase should fulfill at least the following three tasks:

- Offer an opportunity to all participants to share their knowledge, be heard and respected (Berrisford, 2005; Whitney \& Trosten-Bloom, 2003)

- Promote organizational learning by sharing stories of success and identifying forces, factors and strengths that made these performances possible (Ludema et al., 2003)

- Shift participant's attention from the 'problem-focus mode' towards what is working and might possibly work in the future (Whitney, 1998)

It is important to note that it is not the goal to collect objective and correct data at this stage. Thus, the truth is of little interest at this stage. Attention is given to the stories collected, which can be of value for the organization (Cooperrider et al., 2008). The idea to rely on narratives and stories is also put forward in the strategic management literature. Barry \& Elmes (1997), for example, suggest strategic narratives as a vehicle to deliver strategy. They highlight the significance of language, claiming that a "narrative view of strategy stresses how language is used to construct meaning; consequently, it explores ways in which organizational stakeholders create a discourse of direction to understand and influence another's actions" (Barry \& Elmes, 1997, p. 432). Barry \& Elmes as well as the literature on AI suggest utilizing stories to create meaning among participants. Building on this thought, $3 \mathrm{M}$ for example avoids using bullet lists in their strategic plans (Shaw, Brown \& Bromiley, 1998). The authors claim that bullet lists present only an illusion of clarity and therefore recommend a narrative form of strategy presentation. It is argued that the process of packaging a strategy into a story allows the reader to see the reason behind a certain strategy but also sheds light on how he or she can contribute to achieving this plan (Shaw et al., 1998).

Building on an organization's strengths and success-stories, participants are encouraged to dream about what could be (in the future) in the second phase. They are allowed to think big, outside the box and out of boundaries, which may have existed in past (Whitney, 1998). "The purpose of the dream phase," according to Ludema, et al., "is to engage the whole system in moving beyond the status quo to envision 
values and vital futures. It is an invitation to people ... to dream about what their organization could look like if it were fully aligned around its strengths and aspirations" (2003, p. 145). Thus, this phase draws on what has been in the past, amplifies the positive core of an organization in the past and stimulates thoughts, dreams and images about an even better future (Whitney \& Trosten-Bloom, 2003).

While the first stage appreciated the best of what is and the dream stage was about what could be, the latter two consecutive stages (design and destiny) are concerned about the future by making choices for the organization and its members. The goal of the design stage is the creation of actionable possibilities (design elements) through which the dream statements can be brought into life (Berrisford, 2005). As a result of this process, provocative propositions are formulated incorporating the "positive change core into the high-impact processes, systems and programs" (Whitney \& Cooperrider, 1998, p. 20). This phase enables participating employees to reorient and realign themselves quickly to the changing organization (Whitney, 1998). For employees not participating in the summit, the provocative propositions are used to communicate the visions and intentions at a later date (Faure, 2006).

While organizational transformation already occurs in the earlier phases, the fourth phase (destiny) specifically focuses on action planning to ensure further change both at the organizational and personal level (Whitney, 1998). According to Ludema et al. it is a "time for integration, commitment, and focused action. ... It is also a time for seeding the organizational ground of transformation" (2003, p. 203). The overall goal of the Destiny phase is to ensure that the dreams can and will be realized. Personal and/or group commitment to specific action plans and provocative statements are essential since they are a source of motivation to hold on to these statements even if they prove difficult and challenging.

The 4-D model presented is the most common intervention model for conducting an AI-Summit (Faure, 2006). Even though alternative intervention approaches for different business contexts exist, they all build on the same philosophical assumptions as outlined earlier. A good summary of these approaches is presented in Whitney \& Trosten-Bloom (2003). One of these approaches is the 4-I (initiate, inquire, imagine, innovate) model (Watkins \& Mohr, 2001). This approach is very similar to the 
traditional 4-D model and appears to be more "business-friendly" since such emotional words like 'dream' and 'destiny' are not used (Faure, 2006).

\subsection{Limitations and criticism of AI}

Since it contradicts with the nature of AI, discussion of potential weaknesses, shortcomings and obstacles of AI remain silent from AI advocates. In the latest edition of the AI-Handbook (Cooperrider et al., 2008) discussions about limitations and critical aspects in applying AI could not be found in the book (of over 450 pages). Messerschmidt claims there "is an amazing lack of rigorous assessment of AI methodology or techniques ... by its own developers and practitioners" (2008, p. 455).

Bushe argues that positive change, as claimed in the AI-literature, will not appear "without a whole lot of the wisdom of 'traditional OD' applied competently" (2007, p. 35). For example, issues like power and authority are not addressed in the AI philosophy, leaving the door open to manipulate the process or even use it instrumentally (Reed, 2007). Watkins \& Mohr argue from a similar perspective claiming that 'OD-wisdom' in terms of "skills and knowledge are critical for those who want to use AI ... [since an AI intervention is] co-created with the client. There is no AI 'cookbook"' (2001, p. 48)! This does not mean that every AI-intervention needs to be sponsored and facilitated explicitly, but depending on the complexity and change that an intervention is supposed to deliver, the facilitation skills need to be appropriate (Watkins \& Mohr, 2001). To sum up, it is probably naïve to believe that AI without additional 'Organizational Development-wisdom' added would always provide the outcomes that appear to be attributed to the AI intervention alone.

In her article, Mellish (2007) summarizes the findings of analyzing 6 AI cases from a practitioner perspective and provided a list of risks and challenges these interventions faced. These risk and challenges relate mainly to the preparation of an AI summit and the actual summit. Such a list could be used as a source for learning and improving further AI interventions. Adding on to this discussion, Miller, Fitzgerald, Murell, Preston \& Ambekar (2005) put forward the interesting idea that the solely positive focus of AI might be counterproductive. The argument is that in avoiding seemingly negative elements, the AI process can be actually limiting itself since negative 
information and conflict can also be used constructively. As Preston notes "conflict does bring with it good elements such as creativity, unification, and energy, but it is up to the leader to guide it in a constructive direction" (Preston in Miller et al., 2005, p. 104). To sum it up, these few authors critiquing aspects of AI suggest that AI should be more aware of its own deficiencies, acknowledge these and learn from them. Perhaps, as AI builds on its success in the past, it can also learn from previous mistakes and failure in order to achieve better results in the future.

Acknowledging that AI can have potential weaknesses and deficiencies, evaluation approaches can be used to highlight these and also show the advantages and benefits of such AI interventions. In the following, the existing literature on evaluation and evaluating AI interventions is reviewed.

\subsection{Evaluation of Appreciative Inquiry interventions}

The word evaluation is derived from the French verb, évaluer, and means to be worthy or to have value. Roth defines evaluate as to "determine the worth of an effort, or to appraise it" (1999, p. 305).

Although the literature offers a variety of definitions, Preskill \& Catsambas (2006, p. 40) present seven characteristics describing the term evaluation. They claim that most would agree (Note: characteristics ordered as given in source)

- Evaluation is a systematic process.

- Evaluation is a planned and purposefully activity.

- Evaluation involves collecting data regarding questions or issues about society in general and organizations and programs in particular.

- Evaluation is a process for enhancing knowledge and decision-making.

- Evaluation is of critical importance.

- Evaluation concerns asking questions about issues that arise out of everyday practice. 
- Evaluation is a means for gaining better understanding of what we do and the effects of our actions in the context of culture, society and the work environment.

\subsubsection{Objectives of evaluation}

To start the discussion on evaluation, the question why do we need it should be answered. Van der Haar \& Hosking (2004) and Senge et al. (1999) state that gathering feedback is the main objective of evaluation. It is the desire of organization members and practitioners to determine the worth of an effort in order to debate its usefulness and benefits. Organizations and practitioners want to know whether an intervention adds value to an organization and if yes, how much? Roth states "until you get some legitimate feedback that is linked to your activities, you are steering blind, guided by your personal experience rather than the broader organizational information" (1999, p. 304).

\subsubsection{A 'traditional' approach to evaluation}

Van der Haar \& Hosking (2004) refer to the traditional approach of evaluation as 'product evaluation' in which 'pre' and 'post' intervention measurements and a control group that did not receive the intervention are compared with another. Through statistical analysis performed by a detached evaluator, valid and reliable deductions can be generated.

Van der Haar \& Hosking conclude that the 'product evaluation' approach is “inconsistent with a relational approach to AI [... since a] 'product evaluation' approach does not aim to be responsive to multiple local ontologies [and] imposes one reality construction on others" (van der Haar \& Hosking, 2004, p. 1028). Consequently, hard evidence of AI's impacts and results seems to be challenging to gather due to the difficulties in grasping the soft, 'emergent', subjective and positive qualities (Messerschmidt, 2008).

Thus, a difficulty in evaluating AI arises due its underlying philosophical assumptions, which contradict most standard evaluation techniques (Egan \& Lancaster, 2005). They state that traditional evaluation techniques treat "social and psychological reality as something fundamentally stable and enduring" (2005, p. 36). 
This opposes the underlying philosophical assumptions of AI in which reality and meaning is created through social interaction. According to Egan \& Lancaster, AI practitioners and scholars "identify the AI approach as a process focused on the creation and actualization of new beliefs, and provocative propositions" (2005, p. 36). If this statement is seen to be true, standard evaluation techniques may not be capable of 'measuring' such new beliefs and the provocative propositions an organization holds.

Even though difficulties have to be overcome, Messerschmidt criticizes that no practitioners have suggested ways to respond to these contradictions. "Instead have they turned it into an either/or discussion, which is not helpful" and the lack of evaluation becomes "part of the AI 'mystique"” (2008, p. 463). As Rogers \& Fraser conclude "we do not need more narratives of the evaluator [AI believer] as hero" (2003, p. 81), in which the arguments supporting AI rest on hostile evaluations of other approaches (Reed, 2007).

\subsubsection{Existing approaches in evaluating AI}

According to statistician W. Edwards Deming, "97 percent of what matters in an organization can't be measured" (in Messerschmidt, 2008, p. 463), i.e., what really counts cannot be counted. In a similar line of thought, Meador (1999) states that some 'soft' results are almost impossible to quantify. 'Soft' results, like employee satisfaction, diversity or personal change, are factors in predicting things such as organizational change.

The following paragraphs provide some examples of published evaluation approaches. This research is informed and builds on these evaluation approaches in order to:

- Be aware of difficulties that may arise in doing such research,

- Outline the research gap this research aims to address and

- Develop a research design for conducting evaluation in organizations.

Bushe \& Kassam (2005) scanned the literature for published cases of AI and analyzed them by drawing on the secondary data provided in these case publications. 
Interestingly, almost half of the case publications' authors are AI academics. Further more, almost all cases were published by AI academics. All cases involved in Bushe \& Kassam's research were published before 2003. A detailed list of the cases evaluated is given in Bushe \& Kassam (2005, p. 178f).

In the following, a summary of these dimensions and the corresponding results is given.

Table 1: Results of Study

\begin{tabular}{|l|l|l|}
\hline Dimension & Explanation & Result \\
\hline $\begin{array}{l}\text { Transformational } \\
\text { (YES/NO) }\end{array}$ & $\begin{array}{l}\text { If evidence was given of a qualitative shift in the state of } \\
\text { being or identity of the system, a case was coded as } \\
\text { transformational. Could evidence be found that organization } \\
\text { pattern (pre and post intervention) is clearly different? }\end{array}$ & $\begin{array}{l}35 \% \text { of the } \\
\text { cases are } \\
\text { reported as } \\
\text { transformational }\end{array}$ \\
\hline $\begin{array}{l}\text { New knowledge or } \\
\text { New processes }\end{array}$ & $\begin{array}{l}\text { If the intervention geared towards a specific goal and } \\
\text { particular end the case was coded as creating new } \\
\text { processes. If a new way at looking at the world was } \\
\text { accepted and employed some kind of realization, the case } \\
\text { was perceived as generating new knowledge. }\end{array}$ & $\begin{array}{l}\text { Know New } \\
\text { Processes }\end{array}$ \\
\hline $\begin{array}{l}\text { Generative Metaphor } \\
\text { (YES/NO) }\end{array}$ & $\begin{array}{l}\text { Refers to the issue whether symbols (had to be persistent } \\
\text { and evoke a unique shared meaning) that held a meaning } \\
\text { the group members agreed upon were in place or not. }\end{array}$ & $\begin{array}{l}35 \% \text { had a } \\
\text { generative } \\
\text { metaphor }\end{array}$ \\
\hline Figure or Ground & $\begin{array}{l}\text { If the process surfaced some element of the organization for } \\
\text { increased inspection, it was coded as figure. If the process } \\
\text { was able to change or create new background assumptions, } \\
\text { it was coded as ground. }\end{array}$ & $\begin{array}{l}60 \% \text { Figure } \\
40 \% \text { Ground }\end{array}$ \\
\hline Implementation & $\begin{array}{l}\text { A case was coded as improvisation when there were } \\
\text { numerous, diverse ideas for changes pursued by various } \\
\text { actors. Whereas implementation was focused on an end } \\
\text { result that signified termination of the process. }\end{array}$ & $\begin{array}{l}\text { Improvisation } \\
56 \% \\
\text { Implementation }\end{array}$ \\
\hline
\end{tabular}

Source: Adapted from Bushe \& Kassam (2005, pp. 170-176)

All the cases included by Bushe \& Kassam (2005) were considered as successful AI interventions by their authors, even though, as their study shows, only $35 \%$ of all cases analyzed were classified as being transformational. Additionally, Bushe \& Kassam (2005) perceived that only 35\% of the cases resulted in new knowledge. Based on this evidence "it appears that the 4-D process cannot be expected to result in a 'revolution in change' in and of itself' (Bushe \& Kassam, 2005, p. 177). Further on they argue that the act of collecting positive stories about the past might have made 
the implementation of the change process seem to be more feasible, but this "is not in itself sufficient for transformation of large systems as a whole" (p. 177).

Messerschmidt (2008) examined two women's health projects for impacts of using AI as an organizational transformation and team building tool with a focus on evaluating AI itself. In this research, Messerschmidt and his colleagues sought to determine if AI contributed to the achievement of the project goals and, if yes, why and how? When employees were asked about the impact AI had on their organization, they showed enthusiasm, pride, believed in their own institution, and told positive stories of change. When asked for visual evidence showing the impact of AI on the institution 'before-after' stories were presented, i.e. before the intervention floors were dirty, access roads were mired, etc. which has obviously changed. However, Messerschmidt claims that these stories and findings are relative. Without doubt, evidence of positive change is present; yet, whether they really indicate a transformational change is at least questionable (Messerschmidt, 2008).

Nevertheless, the strengths of AI observed in this study are that the AI-intervention promoted positive thought and affirmative action in participant's personal and professional life, that it appears to be an attractive intervention to outsiders and had a spirit-raising effect on participants (Messerschmidt, 2008). On the negative side, running the workshops and training staff was found to be costly and time consuming, especially when the projects continue to be dependent on external practitioners. This leads to the second point on sustainability. Employees who received training in AI were reluctant to pass on their knowledge to colleagues, raising difficulties in keeping up the 'positive spirit' of AI in the long term. As one informant claimed: "When the evaluators are here, AI works; when they are not here, it does not" (Messerschmidt, 2008, p. 460). Note: While these two AI projects were started around 2000, Messerschmidt published his first evaluation report on these two AI projects in 2005 (see Messerschmidt, 2005).

According to Messerschmidt, a problem of relying on success stories in evaluating AI is the chance of being deceived by the 'fallacy of misplaced causation'. He states that "causation implies a strong correlation between one set of circumstances or happenings [before the intervention and inputs] and another [after the intervention and outputs]" (2008, p. 462). Further, he claims that it is certainly plausible that AI 
enhances operations, but there is no proof since it is impossible to directly measure the effects of each input on the organizational transformation. Even so, Messerschmidt (2008) states that stories or other evidence should not be ignored.

The concept of responsive evaluation represents an interesting and relevant approach for this research. Van der Haar \& Hosking (2004) pick up this concept in laying out the theoretical background for evaluating AI interventions. Responsive evaluation aims to have an adaptable design that can emerge during the evaluation process; emphasize the use of qualitative data; report it in a way that the data keeps its richness and diversity so that it allows the readers to make their own judgment based on the data presented. While this approach seems to be more elastic and subjective, it is still the task of the evaluator to design and conduct the evaluation study appropriately.

Given the philosophical assumptions of AI, responsive evaluation "must try to make different constructions explicit and understandable rather than seek to explain and to resolve them in some way" (van der Haar \& Hosking, 2004, p. 1031). In this sense, diversity in the constructions should be preserved rather than reduced. Through storytelling, diversity in the constructions can be enhanced. Additionally, storytelling retains local-contextual details, draws attention to what individuals think the issues are and ensures that evaluation of an intervention is done in context. Due to these characteristics of responsive evaluation, it becomes clearer that evaluation itself is only meaningful to a particular intervention. Van der Haar \& Hosking claim that "evaluation is no longer seen as a program that can be applied universally and that 'starts' at a particular moment - for example, when the AI intervention has ended" (2004, p. 1031).

Reflecting on the above evaluation studies, the authors point out several difficulties and important aspects in evaluating AI interventions. For this reason, the following research needs to be aware of these and address them accordingly. The 20 cases reviewed by Bushe \& Kassam (2005) were all reported as success stories while only about a third of these cases were classified as transformational through resulting in new knowledge. Thus, this research needs to aware that the findings and results of evaluating a particular intervention are likely to be influenced by the evaluator's interests and values (Grant \& Humphries, 2006). 
Messerschmidt (2008) explains that evaluation is exposed to the risk of being deceived by AI success stories. He claims that next to an AI intervention, there are other variables and unknown factors that can contribute to positive organizational transformation. Consequently, evaluation should not get co-opted by 'AI success stories' and instead aim to discover these variables and unknown factors in order to get a fuller picture. Van der Haar \& Hosking (2004) argue that evaluation of an intervention needs to be done in the context of a particular intervention and therefore should have an elastic and adaptable design while ensuring correctness and validity.

Being aware of some difficulties evaluation of AI interventions might face, the three articles also provide interesting questions and valuable implications, which inform this research. The next chapter outlines the research design, how the evaluation of AI interventions is to be performed.

\subsection{The research gap}

The evaluation approaches, as reviewed earlier, give valuable insights into the difficulties that arise in evaluating AI and outline possible approaches on how AI interventions can be evaluated. This research is informed by these evaluation studies while building on them. However, the evaluation approach that will be proposed in this research differs from the reviewed evaluation studies in the following areas.

Firstly, this research aims to consider a broad range of 'outcomes' from the summit. In not predetermining or having expectations on what potential outcomes of AI interventions could be, this research aims to discover and capture a diverse range of outcomes, perceptions and issues. This research is interested in investigating whether or not evidence of change exists in these organizations where this change appears linked to an AI initiative. Finally, the research will aim to provide more data on the basis of which AI's usefulness in achieving the organization's goals and the objectives of performing an AI intervention can be assessed.

Secondly, as noted earlier, the 'timing' of the evaluation might impact the assessment of AI's success, i.e. the evaluation results are likely to be influenced by the time lag between the summit and the evaluation. Yet, the evaluation studies reviewed above, did not consider this aspect in their evaluation designs. Messerschmidt (2008) does highlight it as an issue for future research. This research will set out to address the 
timing aspect (by requiring the summit to have occurred at least one year before to the evaluation starts) in order to be able to draw conclusions regarding the sustainability of AI interventions. Some AI academics point out that AI can result in transformational and long-lasting change (Cooperrider et al., 2008), yet others have raised questions about the extent of such long-lasting transformations (Bushe \& Kassam, 2005). This research is interested in investigating this argument by including a strong focus on the sustainability in the evaluation design.

Lastly, the 'who' benefits question is asked by considering how 'who' is asked might have an affect on the evaluation findings. Bushe \& Kassam (2005) point out that all of their 20 reviewed cases were presented as success stories by the evaluators. However Bushe \& Kassam perceived that only about third of these cases could be classified as 'transformational'. Thus, this research will raise the question whether the issues about 'who' is and appears to be have an effect. This research will, thus, seek to involve summit participants from different management levels of the organization in order to include a variety of perceptions in the evaluation process, making it more robust against criticism. Watkins \& Mohr (2001) point out that AI is capable of engaging a large number of people and creating consistent understanding among them. Thus, including a diverse range of interviewees in the evaluation process allows elaborating on the consistency of their perceptions.

In this literature review, the philosophy of $\mathrm{AI}$ and the call for evaluating $\mathrm{AI}$ interventions are outlined. Further, three different approaches on evaluation showed some practical implications but also some limitations. This research has been informed by these approaches and will seek to build on them. In the following chapter, the methodology for collecting and analyzing data will be outlined in detail. 


\section{Chapter 3: Methodology}

\subsection{Introduction}

In this chapter, the methodology proposed for evaluating AI interventions is outlined. The research questions stated below help to do this purposefully and set out a specific focus of interest in evaluating AI interventions.

\subsection{Research questions}

1. What evidence can be found that Appreciative Inquiry interventions fulfilled the expectations and achieved the intended goals and outcomes?

2. What are the outcomes associated with Appreciative Inquiry summits?

a. Are they sustainable?

b. Are they consistent across different management levels of the organization?

Building on the research by Bushe \& Kassam (2005) and Messerschmidt (2008), this research aims to examine AI's effectiveness in delivering organizational change. In this research, the change that has or has not occurred in organizations will be contrasted with the initial expectations of an organization and the affirmative topic.

Even though it might be a current management 'fad' word, sustainability and the question of addressing it also has its relevance. Question 2.a aims to elaborate on the aspect of sustainability in delivering the outcomes over an extended timeframe. As presented earlier, AI advocates assign AI the ability for transformational change while little research has been performed to further investigate this argument. The question is interested in whether these proposed transformational changes an AI intervention can germinate are sustainable or not.

Question 2.b addresses the argument made by AI advocates (see Cooperrider et al., 2008) that AI has the ability to engage employees on a broad scale and create a consistent understanding among the participants of the summit. As the principle of simultaneity recognizes (see Watkins \& Mohr, 2001), inquiry into something and change are not separate moments. This implies that the process of inquiring alone delivers an outcome consistent and understood across all participants, even if they are 
from different management levels. In order address this question, employees from different management levels are to be interviewed. It is important to note, though, that the interviewees are interviewed on the basis of the same interview schedule independent from hierarchy.

\subsection{Method for data collection}

In order to answer the questions outlined above, two categories of data are needed:

- Data describing the intervention itself, including its goals and objectives - The 'AI intervention detail sheet' will be used to do this in a consistent and structured way. Thus, a detail sheet was used in each case to provide an introduction to the summit, give information on the affirmative topic, the goals and objectives of the summit, the number of employees who participated in the summit, etc. This detail sheet can be seen as a project description of the summit. Since only certain employees in each organization hold this relevant information, the process of collecting this information is less structured. This research will request project documentations of the summit and use this data as a source for the detail sheet. A sheet is prepared for each organization and presented as part of the case analysis in chapter 4.

- Data describing the organization, the intervention and the outcomes of the intervention - Semi-structured interviews are used to gather this data. In doing so, employees from different management levels will be interviewed.

This research is an exploratory study interested in the underlying conditions that may (or may not) contribute to success or failure of an AI intervention. Due to the explorative nature of this research these underlying conditions are unknown at the outset and could differ across organizations. Therefore, a structured quantitative approach to data collection is less suitable since the prior operationalization of all such variables is not possible. Additionally, incorporating local-contextual factors in the data collection and evaluation, as argued by van der Haar \& Hosking (2004), appears to be more difficult when using a quantitative approach.

Therefore, semi-structured interviews are considered as the most appropriate form in collecting this data from employees as they provide a rough structure follow in 
conducting the interviews while allowing the interviewer and interviewee to address topics that emerge during the interview (Eriksson \& Kovalainen, 2008). A semistructured approach of interviewing also supports the claim, made in the literature, to consider local-contextual factors in evaluation (van der Haar \& Hosking, 2004). Thus, interviewees are allowed and even encouraged to talk about their perceptions and provide their stories, experiences and opinions in context to and by referring to the organization. Lastly, a semi-structured approach allows the interviewer to consider and respond to the different backgrounds of interviewees (their involvement in the organization, in the summit, etc.) during the process of interviewing in an appropriate manner (Easterby-Smith, Thorpe \& Jackson, 2008). An outline of the interview questions used in the semi-structured interviews is attached in the appendix (see Appendix C: Interview Schedule).

In the main section of the interview schedule, interviewees are asked to describe the organization with a focus on culture, processes and communication. Furthermore, they are requested to elaborate on how these characteristics changed from before the intervention to now. The interview schedule is designed to 'let the interviewees talk' about how they experienced the summit itself and their experiences in working for the organization before and after the summit. Thus, the questions regarding the organization's culture, processes and communication are purposefully used to get interviewees talking and to avoid using biased or leading questions. Building on the thought of AI, the more positive the questions asked, the more positive the outcome may be, so evaluation has to address this statement appropriately as well. Consequently, from an evaluation perspective, these questions are carefully selected and attempts were made to avoid leading questions.

\subsection{Sampling}

In order to achieve the objectives of this research and answer the outlined research questions, purposive sampling was used (O'Leary, 2004). The process of recruiting interviewees involves the identification of suitable organizations that have undergone an AI intervention. Given the nature of this research, it was aimed to approach organizations possessing the following characteristics. 
- The AI-Summit occurred at least 1 year prior to the interviews. Case studies on AI interventions highlight how excited, engaged, positive participants were in such an intervention (Bushe \& Kassam, 2005). As Messerschmidt (2008) points out, evaluation should not get 'blended' by these short-term 'feelings' of employees and focus on the long-term as well. Since this research is specifically interested in the sustainability aspect in delivering organizational change, a minimum 1-year time-gap between the AI intervention and the interviews was considered to be required for identifying initial trends in the longer term.

- 'Large-scale' interventions are evaluated in this research. This research aims to elaborate on AI's legitimacy and effectiveness in delivering organizational change. Drawing on the findings of a previous research project, $\mathrm{AI}$ is also used in facilitating small group interactions and supporting them in achieving certain objectives. In such small-scale interventions it is more likely to have only a selected group of employees involved (typically senior and top management). Since this research is interested in interviewing employees from various management levels, it was aimed for large-scale (>100 participants) interventions. Additionally, at least $10 \%$ of the total workforce should have been involved in the intervention. This constraint should ensure that the intervention itself is of sufficient 'size' in relation to the whole organization i.e. the intervention can have an organization-wide impact and affect organizational change.

Drawing on what appropriate participating organization should look like, a group of AI practitioners and consultants from Australia and New Zealand were approached and asked to suggest organizations that fulfill the outlined characteristics. Initial contact with these consultants and practitioners was established during a previous research project, in which I interviewed them. Consultants and practitioners were initially contacted based on an AI community contact list published online (Case Western Reserve University, 2010). In total, 16+ consultants and practitioners from New Zealand and Australia were contacted and provided with the relevant information on this research. The majority of them were readily willing to provide their support. Despite this support, a common answer was that they have not been 
involved or are not aware of any interventions with over 100 participants. Another early observation in approaching consultants was that it appeared to be unlikely to find three suitable organizations within New Zealand; hence the need to expand the search to Australia.

Over a period of about three months when contacting consultants, five suitable organizations could be identified. These organizations were contacted using the reference of the consultants. In getting the organizations involved in this study, a number of phone calls and follow up e-mails providing further details on this study were needed. Despite providing further information and explaining the benefit to their organization in participating, two organizations declined their participation, stating the workload of their employees was too heavy or that they were simply too busy at the moment. In the end, the three other organizations agreed to participate in this research.

Once the agreement of these organizations had been gained, the next step included the selection of suitable interviewees. It was aimed to interview between 5 to 7 employees in each organization. In selecting these interviewees, the following characteristics were sought.

- Interviewee participated and was involved in the AI intervention. Participation in the intervention was absolutely necessary since the interview questions focus to a great extent on the intervention itself and its impact on the organization.

- Interviewee joined the organization at least one year prior to when the intervention occurred. Some of the interview questions aim to compare the organization as it was before and after the intervention. Thus, the interviewees should have joined the organization a certain period before the intervention in order to elaborate on what has changed or not.

- Interviewees are a 'representative' selection of the summit participants. Since this research is interested in the consistency of the summit's outcomes among the different levels of the organization, it has to be ensured that the interviewees are selected accordingly. 
The researcher informed the 'contact' person about the specific requirements the interviewees should fulfill. This person then performed the selection and 'recruitment' of suitable interviewees. The 'contact person' is the employee through whom the researcher established first contact to the organization, who supported the researcher in getting the organization involved in this research, and helped in coordinating and scheduling the interviews. It is important to note, the researcher had no influence on the selection of the interviewees beyond indicating the criteria above.

\subsection{Data Collection}

24 interviews were held over a period of about 3 months. As mentioned earlier, three organizations from Australia and New Zealand were involved in this research. The following table presents further details on the organizations involved in this research.

Table 2: Details on the organizations involved in this research

\begin{tabular}{|c|c|c|c|}
\hline & \\
\hline & 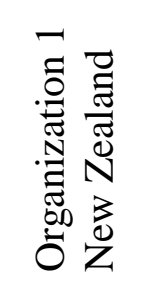 & 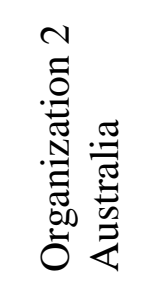 & 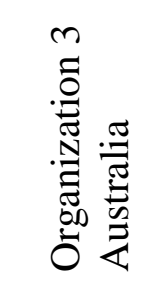 \\
\hline Number of interviews conducted & $9^{(* 1, * 2)}$ & $7(8)^{(* 3)}$ & $7^{(* 4)}$ \\
\hline Date of the Summit ${ }^{(* 5)}$ & $\begin{array}{l}\text { March } \\
2006\end{array}$ & $\begin{array}{l}\text { August } \\
2008\end{array}$ & $\begin{array}{c}\text { February } \\
2008\end{array}$ \\
\hline $\begin{array}{l}\text { Number of people involved in } \\
\text { summit }\end{array}$ & 320 & 150 & 130 \\
\hline Type of organization ${ }^{(* 6)}$ & Profit & Mutual & $\begin{array}{l}\text { Non- } \\
\text { profit }\end{array}$ \\
\hline \multicolumn{4}{|c|}{$\begin{array}{l}{ }^{(* 1)} \text { Interviews occurred across two cities } \\
{ }^{(* 2)} \text { One interview was not recorded } \\
{ }^{* 3)} \text { One interview was conducted with the consultant who ran the summit and } \\
\text { was excluded from data analysis } \\
{ }^{(* 4)} \text { One interview was conducted via telephone } \\
{ }^{(* 5)} \text { Each summit was held over a period of two days } \\
{ }^{* * 6)} \text { All organizations operate in the service industry }\end{array}$} \\
\hline
\end{tabular}

Source: Compiled from data collected

Despite the geographically dispersed locations of the participating organizations, with the exception of one, all interviews were held face-to-face, at the employee's place of work. Therefore, it was necessary for the researcher to travel to the various cities in New Zealand and Australia for data collection. A meeting room or the employee's 
office were the preferred venue for the interviews. In three instances the interviews were held in a café and could be combined with a cappuccino.

Each interview was digitally recorded and ranged in duration from 27 minutes to approximately one hour, averaging at 45 minutes. Before the interview started, the interviewees were given further information on the research and how their interview would be used in this study. In addition, the research agreement (see Appendix B: Research Agreement) was signed and its implications discussed.

Some background information on the interviewees was collected at the beginning of the interview. This assisted the researcher to familiarize himself with the interviewee and ease into the interview process. Some notes were made during the interview, helping the researcher capture the major points and in some instances address these in in more detail. After the interview, the rough and unstructured interview notes were reviewed and written down in a more structured way. In reflecting back on these interviews shortly afterwards, the researcher was able to greater familiarize with the preliminary findings and use this information in the consecutive interviews.

The 'contact persons' and the consultants who facilitated the AI summit provided information on which an AI detail sheet was compiled for each organization. This information was given to the researcher before the interviews in the organizations were conducted and was provided in the form of project documentations, project presentations and reflection documents. The sources used in the AI detail sheets cannot be provided, since it would lead to the identification of the organization. These detail sheets were sent to the respective contact persons for reflection and feedback in order to enhance their accuracy and validity. To prevent confusion with these detail sheets, it is important to note that these sheets are similar to a project description and state the INTENDED goals and outcomes prior to the intervention.

\subsection{Data Analysis}

Figure 4 provides a structure for analyzing and reporting the findings of the interviews. 


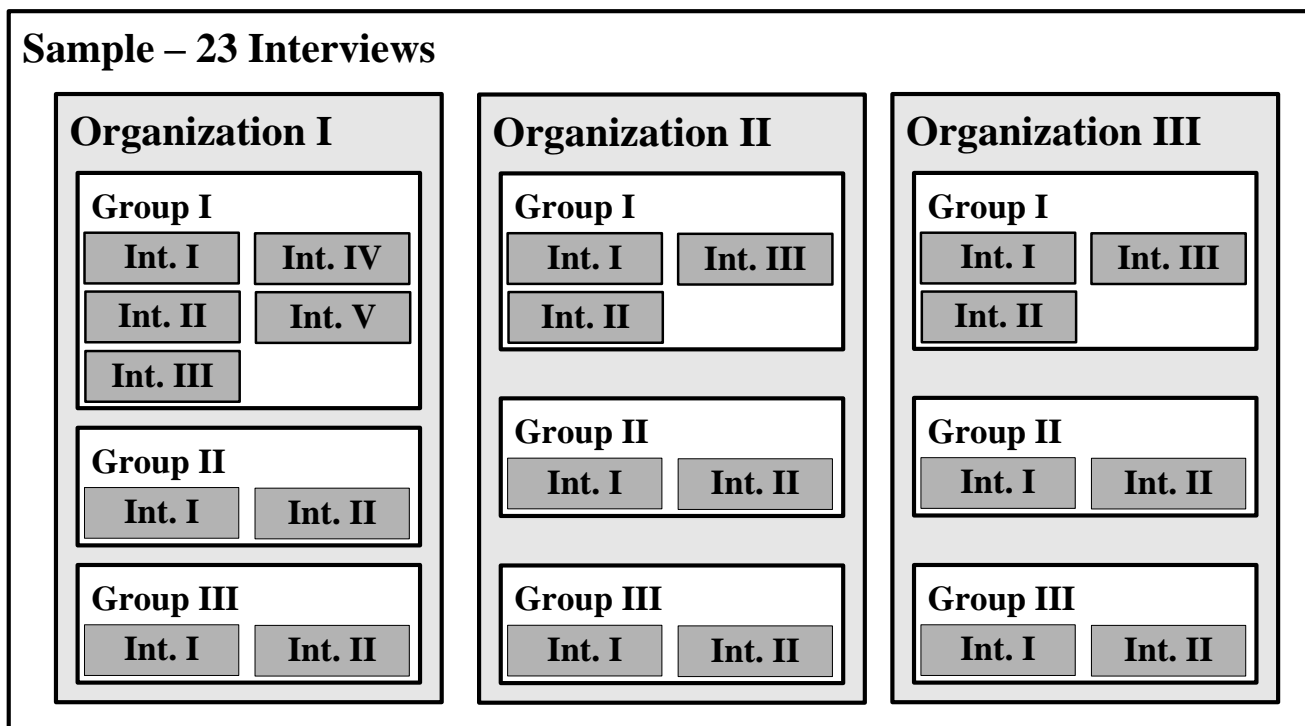

Source: Compiled from data collected

The interviews were held over a period of about 3 months and conducted sequentially.

This gave the researcher the opportunity to focus exclusively on one organization during its data collection, allowing greater familiarization with each case. Additionally, after completing the interviews at each organization three weeks were taken to analyze the data before starting at the next organization.

As figure 4 shows, the interviewees of each organization are categorized into three groups. One research question is interested in the consistency aspect across different management levels of the organizations. Thus, these groups represent the different levels of the organization. The one exception is the third organization, in which the first group consists of external stakeholders to the organization. Since over half of the participants at this summit were external, the researcher perceives that this group needed to be involved. Overall, the interviewees across the three organizations are viewed to be representative of the summit participants.

As a first step in analyzing the data, the recorded interviews are transcribed in full. For greater familiarization the transcripts were read and reread in conjunction to other transcripts from the same group. Following this, the transcripts were coded, with the support of a software application, group by group. As the findings of each group do occasionally show a different focus, the categories used to code data were adapted appropriately. Categories for coding were added and modified during the process as needed. On the basis of the developed categories, the findings were reported groups 
set out in figure 4. This means that between two and five interviews were aggregated at this level of reporting. The approach of reporting data as a group should make it easier for the reader to see the similarities and differences in the findings of the three groups for each organization.

In reporting group findings, the data is presented 'as it is', while analysis and interpretations of the data follows. Abama \& Stake (2001) highlight the importance to make a clear distinction between description and judgment. Furthermore, they claim that the readers should be in a position to make their own judgment based on the evidence presented since "the most important judge will often be someone other than the evaluator" (Abama \& Stake, 2001, p. 10). Considering this claim, extensive use of direct quotes was made and data presented with as little bias as possible.

In a next step, the findings of the three groups are combined and the categories analyzed together highlighting similarities and contradictions among the groups. On the basis of the individual group findings and the combined group findings, the AI intervention of each organization is evaluated by comparing these with the intended goals and objectives as outlined in the corresponding AI detail sheet. Consequently, the discussion elaborates on whether or not and to what extent the intervention can be considered as successful. In addition, the research questions as outlined in the beginning of this chapter are addressed. The legitimacy of this approach for evaluation is supported by van der Haar \& Hosking (2004). They argue for the necessity to perform evaluation in context to a specific intervention, since "evaluation is only meaningful in relation to a particular inquiry" (2004, p. 1031). Therefore, the research questions are addressed for each intervention separately.

In the last step, all three interventions are discussed together, looking for similar and/or contradictory phenomena, patterns and characteristics occurring across these cases. The findings are then compared with the literature on AI and discussed, allowing an assessment regarding the effectiveness of AI to be drawn.

\subsection{Ethical considerations and aspects of confidentiality}

Prior to conducting any interviews, Human Ethics Committee approval was obtained from Victoria University of Wellington. Each interviewee was given an information sheet and a research agreement was signed before the interview started. In addition, 
the interviewees were given an explanation of the implications of being involved in this research and their right to withdraw at any point prior the start of data analysis. The information sheet and the research agreement are shown in the appendices attached (see Appendix A: Information sheet).

The findings of the interviews are reported in an aggregated manner to avoid directly indentifying the interviewee or the organization. The 'contact persons' of the involved organizations emphasized the importance to maintain confidentially and in one instance made their participation dependent on it. Due to the importance of this aspect the researcher undertook the following. Firstly, quotes used in reporting the data were anonymized and company identifiers removed. Similarly, the AI intervention detail sheet provides only limited information on which basis the organization cannot be identified. Secondly, quotes providing information from which the reader could potentially identify the organization from the context were excluded in the reporting of the findings. Finally, the researcher's supervisor monitored the accurate execution of above steps.

\subsection{Validity}

Mayan (2001) makes a distinction between internal and external validity. To be internally valid, the conclusions made in the research must be supported by the data presented. In other words, internal validity is about getting the story right (Mayan, 2001). In this research only a small number of interviewees per organization were involved. Internal validity was achieved in the first instance by reporting the data directly and without interpretations.

A semi-structured approach of interviewing supported the researcher in enhancing internal validity since this gave interviewees an opportunity to 'go off topic' and address a large number of issues. These may appear to be less relevant initially but could have an impact in understanding the outcomes (O'Leary, 2004). Thus, in reporting data, every effort was made to maintain diversity of the perceptions voiced by the participants.

External validity or generalizability refers to the extent "to which the findings of a particular inquiry have applicability in other contexts or with other subjects" (Mayan, 
2001, p. 25). Since the scope of this research is limited to three organizations, the extent to which the results of this research are generalizable is somewhat limited.

\subsection{Limitations}

The suggestion and selection of possible organizations to include in this research represents one possible limitation. Consultants and practitioners may tend to recommend successful over unsuccessful interventions. Additionally, organizations might be more willing to participate in this study if the intervention is perceived as a success. Being aware of this possible limitation, this research might not be exposed to this issue because of the following reason. The criteria set out in this research appeared to not give the consultants much of a choice when suggesting organizations. This implies that they may not have been able to suggest only successful interventions.

The selection of the interviewees performed by the 'contact persons' within the organizations may be another limitation of this research. Due to the involvement of only a small number of interviewees per organization, it is possible that the 'contact persons' show bias in suggesting interviewees arguing in favor of the summit and the organization. Since the interviewees of each organization addressed a diverse range of topics, issues and aspects in regards to the intervention, some confidence could be gained that the selection of the participants did not greatly bias the results.

Since the interviews were conducted between two and four years after the interviewees participated in the AI summits, memory of and recalling aspects that occurred further in the past might represent a potential difficulty for some interviewees. As many of the older details on the interventions were often available in secondary sources, these were collected first and used in the questions to aid recall of specific goals, objectives and summit outcomes. However, this research needs to recognize that interviewees may potentially forget certain aspects that occurred or revise their understanding of those events and outcomes. 


\section{Chapter 4: Findings and Analysis}

In this chapter the findings of interviewing 23 employees of three different organizations are outlined. Each organization is reviewed in three subsections (findings, analysis and discussion). The findings section presents the data of the interviews, which will be condensed further in the analysis section where the data are interpreted. The discussion section answers the research questions based on the data presented earlier.

\subsection{Organization 1}

This organization operates in the banking industry and has over 2,500 employees.

Nine interviews with various staff from this organization were conducted. The interviewees were five branch managers, two regional managers, and two managers working in the call center of the bank. The two regional managers interviewed were the direct supervisors of the five branch managers.

With the exception of BM5, all the interviews were recorded and lasted 44 minutes on average. The following table presents further details on the interviewees of this organization.

Table 3: Details of Interviewees - Organization 1

\begin{tabular}{|c|c|c|c|}
\hline Details of Interviewees: Organization 1 & 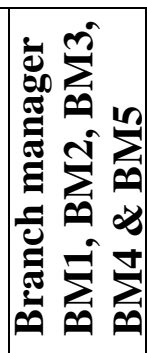 & 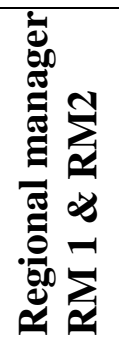 & 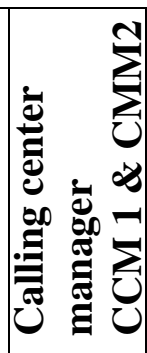 \\
\hline Average number of years in organization & 24.4 & 6.5 & 12.5 \\
\hline Average number of years in position & 9.2 & 2 & 3.3 \\
\hline Average number of direct subordinates & 15 & 18 & 17 \\
\hline
\end{tabular}

Source: Compiled by researcher

The AI detail sheet for this organization was compiled from a project summary documentation prepared by the team of consultants who facilitated the summit. In addition, the contact person of the organization reviewed the detail sheet and confirmed its accuracy. 


\subsubsection{Findings}

\section{AI intervention detail sheet: Organization 1}

$\begin{aligned} & \text { Title of AI intervention / } \\ & \text { Affirmative topic: }\end{aligned}$
$\begin{aligned} & \text { Date of intervention: } \\ & \text { Number of employees involved: }\end{aligned}$
$\begin{aligned} & \text { Number of employees 'impacted': } \\ & \text { cuntomer experience }\end{aligned}$
$\begin{aligned} & \text { Details of Intervention } \\ & \text { Over 2,500 }\end{aligned}$
$\begin{aligned} & \text { Objective: } \\ & \text { The objective of this intervention was to create a clear point of difference and }\end{aligned}$
sustainable competitive advantage through providing consistent and excelling
customer experience. An alignment of the organization's service culture to the brand
and its strategy was sought for. Furthermore, the intervention should provide the
organization with a common strategic framework, a way for communicating and
thinking and assist management and staff in aligning their objectives, prioritizing
competing demands and allocating resources.

\section{Process \& Scheduling:}

Prior to the 2-day intervention in March 2006, approximately 9 months were spent on introducing the framework to the executive teams (involving a series of exploratory workshops) and the design of the implementation framework (scheduling, topic of intervention etc.). After the main intervention, follow up summits were held across the organization in the local branches and are still continuing. Employees are engaged in these summits on a weekly basis around a strategically selected theme. In these engagement sessions (about 30 minutes) employees work on themes for 4 weeks running through the 4 step process of AI (one step each week). Employees of this organization refer to the intervention in 2006 and the weekly sessions by using the term 'WoW'.

\section{Intended Goals \& Outcomes:}

- Build pride and confidence in the brand, and a winning attitude

- Strengthen alignment and collaboration across business units and functions

- Increase empowerment of people by providing them a framework to drive and shape change at all levels

- Shift from a compliance mindset - where sales and service staff were focused upon following process - towards focusing on 'outcomes' with their customers

\section{Interview summaries: Branch managers (BM1, BM2, BM3, BM4 \& BM5)}

In the following, the findings of interviewing five branch managers are presented in several categories starting with 'working for this organization before WoW happened'.

Category: Working for this organization prior to WoW

Branch manager BM4, with over 15 years of work experience at this organization, describes the workplace before WoW as a "not very motivational place to be working 
... the branch environment we were in before was very negative because there was not a lot of celebration, it was numbers driven and it was directive." BM3 claims, "there was no culture" while BM4 states, "I do not think staff got valued."

Communication, horizontally (across the branches) as well as vertically, (across the hierarchy) was perceived as poor and not working (BM1, BM2 \& BM3). BM3 mentions, "I do not believe that people in the head office really understood what was happening in the branch day to day or what staff had to contend with." Due to the lack of communication between the branches "there was no consistency" (BM3). As BM1 puts it, "it was really each manager doing his or her own thing ... we did not have a coordinated approach about how we were going to lifting our performance in customer service, or how we were engaging staff."

In terms of systems and processes BM4 points out that "there was a lot of change going on before ... and it was very much process driven rather than people driven." Similarly, BM3 claims, "we probably have gone like any organization through a lot of systems training and there were difficulties ... the systems were not great, so it is having the challenge there and overcoming that when we are having customer interactions."

Category: The two-day intervention in 2006

The word 'fun' appears 23 times in the transcripts of the branch managers. This is also the word that is most often used to describe the two-day intervention, as the next quote of BM1 illustrates: "Well it was a bit of excitement being involved and the fun we had and the activities for two days were fantastic with a lot of fun." "It was a party, it was great! ... Suddenly to have this group of people that were just basically letting you go for it and being positive was just great" (BM4). The aspect of empowerment was perceived quite positively, as BM3 reflects, "absolutely excited and I felt really energized, motivated in thinking what we could do and great to know that our organization is supporting us." "It was a great turning point because it was something we could focus on as a branch and have an influence over what we could change" (BM2) and "all of a sudden there was that collaboration of what we can do together unified and moving forward" (BM3).

Category: Afterwards - Coming back to the branches 
As outlined in the AI detail sheet, after the two-day intervention, follow up summits were held in all branches across the country for about 30 minutes every Wednesday. While the branch managers were part of the intervention, their staff in the local branches were not. In these 30 minute sessions, every branch worked over a period of 4 weeks on a topic (every branch had the same topic at the same time).

BM1 reflects, "the challenge was of course after these two days to engage and bring the rest of the staff on-board and trying to get the same level of excitement and enthusiasm." The branch managers reported several difficulties they had to overcome in getting staff engaged and contributing to these weekly sessions. BM4 reflects, "for some people this whole WoW culture was a struggle because they have been so used to just being directed." BM3 highlights the importance of having fun during these sessions and points out that "not everybody can facilitate a fun session." Thus, facilitating these weekly sessions was sometimes not an easy task for the branch managers (BM1, BM3 \& BM4). BM4 experienced resistance to change by the staff, pointing out that some staff have gone through a lot of changes in the past and might have felt that this is just one more thing that will disappear. Still, "sooner or later everyone had to buy in because after six months it was not going away, it was still here. There was no way to get around it" (BM2). After time passed by and "without them even realizing, the whole way we worked as a group changed ... and everything was more done as a group rather than an individual" (BM4).

Category: Four years later - Now

BM1 summarizes, "the intervention has not changed business dramatically. It has probably changed the culture, has given us some direction and helped us to move forward as a unit." BM2 perceives that the biggest impact has been "with the customer experience. The experience we have with the customer and we put more of a focus on our customer ... we are more aware of our customer responses rather than what we think our customers say." Providing a more consistent and better customer experience was perceived as one major outcome of the intervention (BM1, BM2 \& $\mathrm{BM} 3)$.

Another outcome of the intervention was noticed in the staff area. BM2 says, "there was also a big shift around the empowerment of staff ... I think initially it empowered 
the staff to actually look at themselves and what they can do themselves to actually be fully engaged about coming to work at the bank. It made them fun."

BM4 notes, "I think the expectation is now that the weekly sessions are here to stay and that the way we interact with our customers is that we are here to serve our customers and do what is liked by the customers and the whole culture has changed."

\section{Category: Sustainability of AI}

In the following, statements relating to the aspect of sustainability of AI in this organization are presented. BM1 puts it this way: "it is four years down the track now and I guess if feel now the same than I felt two years ago. It is just part of our business, is part of what we are doing on Wednesdays, it is fun, it is enjoyable ... We have done it now for four years and it is not running out of steam at all. I still find it effective, staff seem to be engaged around it and it is part of how we are doing our business from Monday to Friday." This statement generally summarizes the overall perception of the branch managers on WoW.

Branch managers $\mathrm{BM} 1, \mathrm{BM} 2$ and $\mathrm{BM} 4$ point out that in their branches there was a perception from staff that WoW would last only for a while and it would go away. "But it did not go away really. I guess it got stronger and stronger and became more fun and became part of our normal week" (BM1). "After six months it was not going away it was still there" (BM2). BM4 states, "it became part of all our communication, it became our language, and even for our customers it has become language." "I believe it is embedded so much, you ask any staff member and they can tell you what it is about" (BM3).

Even so, most of the branch managers argued WoW is embedded in the organization, and branch manager BM4 highlights the importance of re-energizing it, claiming, "from a leadership point of view, we need to be re-energized occasionally as well. So whilst it is embedded in the culture, I think it still needs to grow ... it needs to be reenergized and to involve other people on a regular basis as well. So it is not the same people sending the same message. It is good to have different people involved."

Category: Consistency 
The branch managers claim that WoW supported the organization to deliver a more consistent customer service in two ways. Firstly, consistency is enforced among the branches by having weekly sessions with the same topics (BM2, BM3 \& BM4). BM3 points out that, "everybody throughout the country would be running a weekly session, so you knew that ... everybody is on the same page." BM2 claims, we know "that there is uniformity across the network of all the branches. We are all trying to get out the same message to the staff and customers."

Secondly, consistency is reinforced within the branch by its employees. BM3, BM4 \& BM5 report that existing staff are looking for consistency, which for example has an impact on hiring new employees. BM3 explains, "every time when we keep staff, that is immediately what [existing staff] are looking for in that person. Even though a new person has not had the WoW culture embedded in them that is what [existing staff] are looking for." "I suppose that is why we are so particular with when we do get new people that we look at them as to how they would fit into our culture. I think that is really important" (BM4).

\section{Category: The impact of AI}

This category summarizes the positive outcomes of WoW. As already mentioned in a previous category, the biggest impact of AI is perceived to be in the customer service area (BM1, BM2, BM3 \& BM5). BM1 states, "it had a significant impact on us, lifting our overall customer service and outperforming in some cases simply because it motivated people." The aspect of increased motivation of staff due to the intervention was also shared by BM4, arguing that empowerment, acknowledgement and recognition of staff had a positive impact on them. "And if you create a culture within the bank so that staff enjoy being here and enjoy working here it can only result in running a better business" (BM3).

For BM1 the weekly sessions are also a tool to enhance communication within the branch and gather feedback, stating that "you know how they are doing out there; you can see if there is any frustration, any issues or obstacles that might be blocking their ability to do their job better. They will come out on Wednesdays ... it has been such an effective tool for managers even just to hear what staff are saying." 
Empowering staff to actually facilitate the weekly sessions by themselves resulted in a few other, possibly unintended, positive outcomes. BM4 states "it is actually quite interesting when someone is running a session, everyone is supportive. Rather than the manager doing it, it is one of their peers who is leading this. It is great. It gives them ownership ... by having [staff] actually lead it, it means that we are saying that everyone can be a leader in this organization." At this point it should be mentioned that the facilitation of the weekly WoW sessions was initially only the branch manager's duty but in most cases, after a few months staff took over that role. Another outcome of empowering staff to facilitate the weekly sessions also "helped to make some people more confident. People you would not have thought would have contributed, have got up in front of the group and presented and some of them have been quite outstanding. You would not have thought they have the skills because they would not have had these opportunities in the past" (BM1). BM1 \& BM5 claim that the WoW sessions facilitated by the employees are still a great opportunity for staff to develop their skills further.

\section{Category: Difficulties for managers and staff}

This category summarizes some difficulties managers and staff faced in integrating WoW into the branches. One challenge was simply resistance to change. BM2 points out that some "staff were stuck in the past and did not want to move." BM4 claims that staff "had been pushed into a lot of change over the years, which I would not have said was good change. So this was another thing we were going to do. We had to get past this to prove to them it was good change as well." BM4 reflects that the 'engaging' style of leadership was difficult for some staff to buy into, because they have been used to being directed.

Not only for staff, but also for the branch managers, the weekly sessions were something different and challenging to do because "not every manager would have the capability of leading a WoW session how a WoW session should be led" (BM3).

An interesting result in interviewing BM4 was the preference of having a higher staff fluctuation. BM4 points out that new staff can change the whole dynamic of the group in the weekly sessions and unfortunately "I am not getting fresh people coming through ... In some ways you have got the experience. Experience is good if it is used in the right way." 
Category: Impact of leadership - the case of BM5

In an impressive manner, BM5 illustrates how one employee can have a major impact on the performance of a branch. At the time BM5 joined a branch in the position of branch manager, this branch was not performing very well. More specifically, the branch belonged to the $10 \%$ of the lowest performing branches in this organization. Within one year, the branch was turned around by BM5 and was the best performing branch in its region. Within another two years, this branch finally was the best performing branch in the country. Since the performance of this branch started to improve about a year before the AI summit occurred, this change can be directly linked back to BM5's abilities to make this turnaround happen.

An interesting answer BM5 gave was that the ideas and concepts of WoW "were not something really new to me" and "were already here." Consequently, WoW was not really something fundamentally new to the branch and its employees. Still, BM5 appreciated WoW with its weekly engagement sessions and the support from top management because it created uniformity around the brand. This example shows to what extent a branch manager can have an impact on the branch. While WoW supports the branch managers to engage employees and to improve the branch performance in the long-term, the branch managers play an important role. BM5 concludes, "I think a lot depends on the branch managers. I think you have to believe in it."

\section{Interview summaries: Regional managers (RM1 \& RM2)}

Category: Working for this organization prior to the AI intervention

Similarly to the branch managers, the regional managers describe the organization before WoW as a place where "staff morale was not that high" (RM1), without "positive brand presence" (RM1), "poor customer satisfaction measures" (RM2) and poor staff engagement (RM1 \& RM2). Regional manager RM1 claims, "the most celebrated measure across the organization was cost to income ratio. Nobody talked about customer satisfaction, market share growth or any of those sort of key performance measures."

Another aspect both regional managers mention was the timing of WoW. RM1 states, "the way it was launched was exactly what needed to happen ... The timing as right." 
"Because the organization needed to make changes, there was an appetite to do things differently and try things to turn things around" (RM2). "The timing of WoW coincided with a number of other things going on. We had come through a very fantastic financial period ... the economy was flourishing ... we were getting a lot more investment and support at the same time ... it was almost the perfect storm in a positive way" (RM2).

Category: The two-day intervention in 2006

The event was "a really exiting couple of days, it was awesome" (RM1). "The event was fantastic - just what we needed" (RM2). Both regional managers highlight the commitment from the organization and management to WoW. In terms of management commitment, RM1 claims "the key to it being successful was it was very much leadership-led. So our managing director and the executive team were all on board with it, they were leading and driving it." RM2 highlights the organizational commitment to WoW, "we were not just talking about it, it was not just lip service - it was real," recognizing the huge "investment that was required to make that happen."

Category: Afterwards - Coming back

After the intervention, the task for RM1 was to support the branch managers in "taking WoW back to their staff." RM1 reflects, it was about giving the same messages they received at the summit to their local employees at the branches "so that is about making you feel important and recognized." In doing so, RM1 let "the branch managers decide on something exciting to launch it to their staff that they thought their staff would get the most out of it."

RM2 states that the leadership team also spent a lot of time talking about stories and sharing stories, "because we wanted to recognize the best stories, you then start putting processes in place to evaluate and recognize those stories that stand out over others." Thus, the leadership team supported the branch managers in putting processes in place to recognize and value good stories from the branches.

Category: Now, the future of WoW and its sustainability

"If WoW has done anything then it has created a positive culture" (RM1). "I think it has been the most fantastic thing the organization could do ... we have got meaningful engagement from all areas around this and it is a great achievement ... I 
suppose in many ways it has transformed our culture" (RM2). "It is involved in everything we do" (RM1).

In asking the regional managers about the future of WoW, RM1 states, "I am confident that it will continue going as it is going" because "the reality is even if you took it away, the word WoW or the Wednesday meetings, it would still happen. So you cannot take it out of people, it is now the way that we do things ... it is very engrained in what we are doing now."

\section{Category: Consistency}

"WoW was something everybody across the country could identify with, so it is a consistent approach really ... WoW enabled that actually everybody in the country is talking about the same things" (RM1). Commenting on these two statements of RM1, it is important to consider that the term 'everybody' addresses employees from the branch network. RM2 participated in the intervention in 2006 but worked in a business unit outside the branch network at that time. Since WoW was designed mainly for the branch network, it raised the questions for RM2, such as "how does this work in my environment? How does this become relevant to me"? Consequently, RM2 faced some difficulties in making it work within the team but "could see the importance of being aligned to this."

Similarly to what the branch managers said, RM2 perceives that WoW "is also infiltrating our approach to recruitment. So I guess we are looking for WoW staff when we employ them so we have got a better idea of the type of people we want to bring into the business." Consequently, when recruiting new staff, consistency and alignment to WoW and the culture is sought after.

Category: Perception and meaning of WoW for regional managers

In transcribing the interviews it appeared that WoW had a slightly different meaning and use for the regional managers in comparison to the branch managers. Especially, business strategy and the support of WoW in communicating strategy turned out to be more important. RM1 describes one of the tasks to be "able to look at what we need strategically as a bank in terms of our future and also dealing with the operational, day-to-day sort of stuff, and filtering that through to the branch managers to make it applicable and easy for them." "We are clearly recognizing that the world is changing 
around branch banking ... so we have to engage customers differently, we have to change our operating model and those sorts of things. WoW is going to be important in the way we help our staff understand what changing needs customers have. So there is a much stronger focus on customer education" (RM2). RM2 continues, saying "it is not what do we do with WoW but how do we use WoW to help us do the things we are trying to do in the business." Building on these statements, WoW also represents a tool for communicating strategic intentions from top management, as regional manager RM1 explains: "The executives decide on the topics that are most important to us at the moment and what themes we are going to deliver out." Afterwards an internal team works on further details around the themes before it goes out to the branches as guidelines to focus on in their weekly WoW sessions. To conclude, RM2 describes WoW as an "emergent cultural program that was customer centric and really strongly supported change management strategies."

\section{Interview summaries: Call center managers (CCM1 \& CCM2)}

Category: Working for this organization prior to the AI intervention

In a similar tone to the branch and regional managers, the call center managers describe working for this organization prior to $\mathrm{WoW}$ as quite different to what it is now. CMM1 states, "communication was poor and it was definitely poor between the business units but it was poor overall" and claims that the different business units cared just about their own little world, not about the customer nor about the organization as a whole. "You did not have that collective strategy around the customer, you did not have that family as one. You were all separate business units" (CCM1). It was also "very focused on driving revenue, the business outcomes around that and customers were not at the front of decisions. So we did things for revenue rather than thinking about the customer experience" (CCM2). Additionally, "hierarchy was quite important, it was the general manager who had the final say and also directed the business" (CCM2).

Category: The two-day intervention in 2006

The contact center managers also reflected on what happened at the intervention very positively. "The feeling was amazing to be part of this ... that you are treated in such a nice way and it did make me feel quite special ... it was amazing after the 2 days, how positive you felt about the business and wanting to be a part of it" (CCM2). 
Building on the point CCM1 raised earlier that the business units worked very independently from each other, CCM1 states, "it was a nice way of bringing everybody together ... by bringing WoW in, it got us all thinking as one" (CCM1). By having all the managers in one place, CCM2 appreciated the opportunity for networking with various branch managers, which was of particular importance in the role $\mathrm{CCM} 2$ was in at that time.

Both managers also experienced the commitment from the organization and management to do this, because "you could see that the bank had invested a lot of money in this and they really wanted it to work" (CCM1). "It was amazing to me that the business would spend that much money on bringing people together ... I potentially thought, what is the value of spending this much money and are we going to get an outcome for the amount of money that we spent" (CCM2).

Category: Afterwards - WoW with new staff

The findings in this category differ significantly between the two call center managers due to the different circumstances they were in at that time. Thus, in the following, the 'story' of CCM1 is presented. CCM1 was given a task to create a new division within the contact center business unit. CCM1 remembers having almost no difficulties in rolling out the concept of WoW to the staff because "I was lucky that in my area, they were new staff ... and I was able to sell them properly what the concept was about, what they could get out of it and they do not know any different." Consequently, WoW was just seen as being part of the business and the way things were done, resulting in little resistance from staff because they just did "not know any different" (CCM1).

Category: Afterwards - It has to be customized

This category reports the 'story' of CCM2. "The target audience for WoW was very much the frontline ... it is not focused on product managers and marketing managers and product development" (CCM2). Since CCM2 worked at that time in the product area the question was how "could we make WoW work within the product environment and how would we make it work?" In agreement with CCM2's manager at that time, WoW was not "something that would have been appropriate for product in that shape of form" but we "definitely have to understand what it is and how to use it." 
About a year after the launch of WoW, CCM2 joined a division in contact center, reporting "we tried to launch it in the contact center before I came in and it was not successful ... it fell over very quickly at that time ... did not keep continuing." As reasons why it did not work in the first place, CCM2 reflects, "people did not buy into it, there was no structure, there was no clear owner and there was not the team to drive it." Another difficulty was the topics of the weekly WoW sessions being "very much targeted on branch, not contact centers. Very branch specific." As CCM1 already pointed out, WoW is very much focused on the front line in the branch network. Thus, when WoW was launched, it "did not quite match into what it was in the contact center" (CCM2). "We tried to pick up a branch focused initiative and put it straight into the contact center having different staff members and different job roles ... [we] did not put enough thought behind ... how we can make this work" (CCM2). As a result, the initial launch of WoW and the weekly sessions did not last very long.

In launching it again, CCM2 states "I really went through a full sort of analytical sort of phase and investigated how can we make it work and really broke some sacred cows", highlighting the importance of detailed planning beforehand while facing some resistance in re-launching WoW. Further on, CCM2 states the themes for the weekly meetings were quite different in the beginning to what was needed in the contact center. Thus, "we actually changed them so that they were applicable to contact center because sometimes they were not."

\section{Category: Now}

Focusing on now, CCM2 claims "WoW is going well. It is probably not where it was when we first launched it. I do not know if it needs to be there, where it was, because we have a much greater focus on our customers now." The only drawback CCM2 mentions is that "it becomes sort of institutionalized ... it does not have the passion or the follow through. It is just something we have to do because it is a tick in the box." As a consequence CCM1 and CCM2 claim it has to be re-energized.

CCM2 summarizes, WoW is "a vehicle to get a message across ... what we are trying to drive ... it has given my team energy, it has bonded my team; they work together a lot better now ... it was really a vehicle to bring the team together." WoW made "our people feel like they are part of our organization collectively, because we are all 
singing the same tunes." It also changed our culture, "the culture is I am a blue person, I am blue at heart we call it" (CCM1). Still, CCM1 also believes that "there is not enough momentum anymore to make sure these WoW sessions are happening" in all the business units.

Category: Meaning of the intervention for CCMs

For CCM1, WoW is also a tool to communicate to staff, claiming that "there is a serious message behind all the weekly issues, but if you do it in the right way and play and have fun, you can still get it across to your people."

CCM2 points out that WoW gave staff an opportunity to learn how to facilitate and lead weekly sessions. Consequently, these additional skills allowed some staff "to move on through the organization and not just into team leader roles but also into my team."

\subsubsection{Analysis}

The objective of this analysis section is to provide the reader with a compact summary of what the interviewees have said so far while pointing out similarities and contradictions among these three groups of interviewees.

\section{Category: Working for this organization before WoW}

The perception of the organization in terms of people, processes, communication and culture prior to WoW across the interviewees is very similar. Communication was perceived to be poor (BM1, BM2, BM3 \& CCM1). BM4 described the workplace at that time as a "not very motivational place to be working" with poor staff engagement (RM1 \& RM2). In terms of structure and processes, interviewees reported a very independent way of doing business (BM1, BM2, CCM1, CCM2 \& RM2) claiming we "did not have that family as one" (CCM1). Poor communication and the fragmented way of doing business might have caused inconsistency in delivering customer service, as mentioned by BM1, BM2, BM3 \& CCM1. The culture of the organization was perceived as having poor staff engagement (RM1 \& RM2), emphasized hierarchy and directive (BM4, CCM2), having no culture, (BM3) nor a sales culture (RM1) and "process driven rather than people driven" (BM4).

Category: The two-day intervention in 2006 
The interviewees described the intervention as fun to be at (BM1, BM4, RM1), great (BM4), amazing (CCM2), exciting (BM1, BM3, BM4, RM1, RM2), energizing and motivating (BM2, BM3) and making you simply feel special (BM3, CCM2). Additionally, by bringing everybody together, people who may have not known each other before started to engage and collaborate, (BM3, CCM1, RM1, RM2) which in turn created an opportunity for networking (CCM2). CCM1, CCM2 \& RM2 also recognized the fact that the bank had invested a lot of money in this and therefore could see the commitment of management and the organization to it.

\section{Category: Afterwards}

After the summit it was the task of the branch managers to "engage and bring the rest of the staff [in their local branches] on-board" (BM1). In doing so, the branch managers faced difficulties in facilitating the weekly WoW sessions (BM1, BM3 \& BM4), getting staff on board and contributing (BM4) and simply convincing staff that WoW is a change for the better. RM1 points out that the branch managers could decide on how to launch WoW so that staff could get the most out of it. Having this leeway in launching it while dealing with some difficulties as outlined earlier put the branch managers in a key position in making WoW work. Even though the branch managers got support from the regional managers, it was their knowledge about the branch and their leadership skills that were needed to address certain issues adequately. BM4, for example, states that due to the characteristics of the branch it was a "matter of slowly getting people on board." Consequently, the successful launch of WoW in the branches resulted not in itself by using AI; it was also the knowledge, skills and abilities of the branch and regional managers, which contributed to that.

In contrast to the local branches of the branch managers, the call center department in which CCM2 works failed initially in integrating WoW. WoW "fell over very quickly ... did not keep continuing" (CCM2) due to the lack of staff buy in, clear structure and ownership. Additionally, the weekly WoW sessions were "very branch specific" and focused on the branch network and "did not quite match into what it was in the contact centers" (CCM2). After interviewing 9 employees of this organization in total, this is the only instance in which WoW did not keep up its momentum and fell over. It is likely that a lack of customizing of this branch-focused initiative might have caused 
the initial failure. CCM2 states, we "did not put enough thought behind it" initially and so "I really went through a full sort of analytical phase and investigated how we can make it work" for us.

CCM1, on the other hand, did not face difficulties in sustaining the weekly WoW sessions simply because CCM1 created a new team within the call center around the time the summit happened. According to CCM1, it was easy to 'implement' the concept of WoW and the weekly sessions because "they did not know any different." Keeping this point in mind, BM4, working in a branch located in a more rural area, wishes to have higher staff fluctuation. "You are not getting fresh people coming through" in this branch and "a couple of new people could change the whole dynamic again, which is great." Additionally, BM1, working in a branch located in the CBD, appreciates "having staff coming in from other branches ... and having a good balance of staff." These three interviewees indicate that staff movement and "getting fresh people coming through" (BM4) tends to support the process of establishing and sustaining WoW.

Category: The outcomes and results of WoW

Culture - "If WoW has done anything then it has created a positive culture" (RM1). "It has transformed our culture" (RM2). Additionally, BM1, BM2, BM3, BM4 and CCM1 highlight that WoW changed the organization's culture for the better. BM5 and CCM2 did not mention the word culture explicitly but also acknowledged the positive outcomes of it. In the following, several brief statements are presented, describing what a 'more positive culture' means to the interviewees. BM1 states WoW "has helped us to move forward as a unit." "We are all singing the same tunes" (CCM1). "It empowered the staff" (BM2). "It made them fun" (BM2). "We have got meaningful engagement from all areas around this" (RM2). It "has bonded my team, they work together a lot better now" (CCM2). "It motivated people" (BM1). "When someone is running a [WoW] session, everyone is supportive" (BM4).

The interviewees suggest that this new culture affected the organization in two ways. Firstly, this new culture in combination with the weekly WoW sessions had a strong influence on how the employees interact with customers. BM2 explains that the employees are more aware of the customer responses. BM1 reflects, it helped us in "lifting our overall customer service and outperforming in some cases." Arguably, to 
put the improved customer service down to a better culture exclusively might be oversimplifying, but culture definitely contributed positively as BM3 states, "if you create a culture within the bank so that staff enjoy being here and enjoy working here it can only result in running a better business."

Secondly, culture appears to have an impact on hiring new staff. RM2 states, it "infiltrated our approach to recruitment ... we are looking for WoW staff when we employ them" (RM2). BM4 claims that we look at new staff "and how they would fit into our culture" because existing staff simply expect and demand this kind of attitude towards work from new staff as well.

Strategy communication tool - WoW also represents a vehicle for communicating strategic intentions and supports change management strategies (RM2). In particular for the regional managers, WoW enables staff to better understand the changing environment this organization operates in and what the implications for customer service are. CCM1 recognizes, "there is a serious message behind all the weekly issues" and you can get this across to staff if you do it the right way.

Staff development opportunity - A probably unexpected outcome of running the WoW sessions is that the weekly sessions facilitated by staff give staff an opportunity to develop their skills further. BM1 reflects that staff enjoyed having the opportunity to present in front of the group and some of them did an amazing job. Thus, the weekly sessions do not only allow staff to practice their facilitation skills but also give them an opportunity to be recognized by their supervisors as possible candidates to move on in the organization into team leader and further roles.

Category: Sustainability of outcomes and results

BM1 summarizes, "it is part of our business, is part of what we are doing ... it is not running out of steam at all. Still find it effective, staff seem to be engaged around it ... became part of our normal week." The other branch managers support this perception of BM1 to a great extent. Even so, CCM2 claims that since WoW was launched, it lost some of its momentum. It might be at risk to become institutionalized and just something the organization has to do because it is a tick in the box. BM4 supports this claim, highlighting that it needs to be re-energized occasionally as well. To summarize, the perception of the interviewees is that WoW is part of the way business 
is done in this organization now but they also clearly recognize the need to constantly reinforce it in order to keep up its momentum.

\subsubsection{Discussion and summary}

After having reviewed and analyzed the interviews, the question of whether the intervention delivered the outcomes that it was designed for, will be addressed next. In alignment to the first research question, evidence for change as presented above, will be compared with the intended goals and outcomes (as presented in the AI detail sheet). Additionally, consistency of these outcomes across different levels of the organization and the sustainability of these outcomes will be reviewed.

As presented in the AI detail sheet, the first intended outcome of the intervention was to build pride and confidence in the brand, and a winning attitude. In reviewing the interviewees reports about taking part in the summit, the findings show every interviewee enjoyed taking part. CCM2 states, "the feeling was amazing to be part of this ... that you are treated in such a nice way and it did make me feel quite special ... it was amazing after the 2 days, how positive you felt." This statement strongly indicates that the summit made CCM2 more proud of being part of this organization. "To have this group of people that were just basically letting you go for it and being positive was just great" (BM4). This statement may not indicate that confidence was increased automatically. Even so, this statement acknowledges that BM4 enjoyed the loose way of engagement, allowing the discovery of the possibilities, opportunities and strengths this organization had, which in turn may have had a positive impact on confidence in the organization.

While the summit tends to show general consistency of these findings among participants, the 'wider' organization, including employees not participating in the summit, is considered in this discussion. Reflecting on the findings on what happened after the summit, it was a challenge for the interviewees to bring it back to their branches and teams. The fact that the weekly sessions are still happening in the branches and that staff seem to enjoy WoW indicates that staff appreciate the message WoW is sending out. Whether this increased staff's pride and confidence in the brand cannot be confirmed or negated since it was not possible to interview all levels in this study. Still, the interviewees feel quite positive about the involvement and 
engagement of their staff, indicating a positive impact on the pride and confidence of staff in their organization.

Did WoW strengthen alignment and collaboration across business units and functions? The summit allowed the participants to work with colleagues across the organization, and also allowed them to strengthen their networks within the organization. This gave interviewees the opportunity to get to know the different parts of the business and see how they relate to each other. As CCM1 points out, "it was a nice way of bringing everybody together ... it got us all thinking as one" indicating that the summit enhanced collaboration and created alignment.

Post summit, the weekly WoW sessions strengthened collaboration and alignment further in two ways. Firstly, the session topics were the same across the whole organization, enhancing consistency. "Everybody is on the same page" (BM3) because "there is uniformity across the network of all branches. We are all trying to get the same message to the staff and customers" (BM2). Secondly, WoW provided the interviewees with a platform to talk about the business. WoW became a common theme everyone in the business could talk about and relate to.

This discussion indicates that the WoW initiative contributed in achieving this goal in the short-term as well as the long-term. The summit itself brought the employees together, engaged employees from the different business units and fostered collaboration. Further, the weekly engagement meetings post the summit continued these developments in making the employees work on similar themes, which created alignment across the branch network of this organization.

Another goal of WoW was to increase empowerment of people by providing them a framework to drive and shape change at all levels. BM2 confirms, "there was also a big shift around the empowerment of staff." Further on, BM2 explains that staff needed to be empowered and given the permission to 'WoW the customer' without having to fear consequences in doing so. If for example, a customer wants to have some fees waived staff need to know that they are allowed to do that (BM2). Additionally, by letting staff facilitate the weekly sessions, it gave them control over what is actually happening in these sessions. Even though the weekly topic itself was set, staff were empowered to plan and conduct the facilitation in order to deliver the 
best possible outcome. Consequently, WoW gave staff ownership of the sessions and empowered them to contribute and drive change within their working environment.

The last and probably most important goal of doing WoW in the first place was to facilitate a shift from a compliance mindset towards focusing on 'outcomes' with our customers. In reviewing the data presented on this case, it appears that this last goal of WoW resulted as a consequence of achieving the first three goals presented above. Empowerment of staff allowed and encouraged staff to work in the best interests of the customers and be proactive rather than reactive in their customer service. Alignment and collaboration across business units removed this siloed way of customer interaction. The focus of doing what is best for the customer in your business unit changed to what is best for the customer by referring and offering services across different business units i.e. it does not matter where (business unit) and how (channel) the customer does business with us as long as they actually do business with us. Pride and confidence of staff in the brand might have had a positive impact on how staff interacts and services customers.

The staff interviewed in this organization confirms that WoW contributed in enhancing the level of customer service. CCM2 explains that the organization now has a stronger focus on its customers, while BM1 confirms this, claiming WoW helped the organization in lifting its overall customer service.

To conclude, this discussion suggests that WoW delivered the outcomes it was initially designed for. Additional, to the desired outcomes, WoW also caused some positive side effects - e.g. staff development opportunities, impact on recruitment of new staff and the creation of a customer focused service culture. Likely as a result of all the achievements and successes of WoW, the organization is committed to continue the weekly engagement sessions in order to ensure that these goals and outcomes are also achieved in the future. 


\subsection{Organization 2}

This organization also operates in a service industry, employs about 1200 staff and is a mutual in its nature (i.e. the members of the organization are also the owners).

Seven interviews with various staff of this organization were conducted. In addition, an external Appreciative Inquiry and change management consultant who was actively involved in the project activities and facilitation of the summit was interviewed. The findings of the consultant's interview were used only to gather some background information about the organization and not included in the analysis and discussion of this case.

The interviewees were three staff members, two senior managers, one general manager and one executive general manager. The general manager and executive general managers are both referenced with the term general manager in order to ensure confidentiality among staff in the organization. Senior manager 2 was involved in the project activities prior to and post the summit but only attended the second day of the summit due to personal circumstances. All the interviews were recorded and lasted 37 minutes in average. The following table presents further details on the interviewees of this organization.

Table 4: Details of Interviewees - Organization 2

\begin{tabular}{|c|c|c|c|}
\hline Details of Interviewees: Organization 2 & 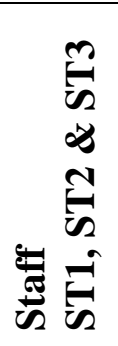 & 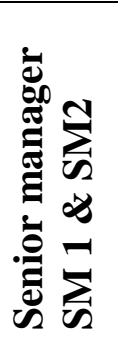 & 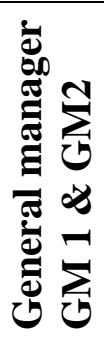 \\
\hline Average number of years in organization & 7.7 & 6 & 10.5 \\
\hline Average number of years in position & 2,3 & 1,5 & 1 \\
\hline Average number of direct subordinates & 2,3 & 0,5 & 6 \\
\hline
\end{tabular}

Source: Compiled by researcher

The AI intervention detail sheet for this organization was compiled from two Power Point presentations (leadership briefing on the summit \& a presentation summarizing the outcomes of the summit) and the interview with the consultant, who was in charge 
and facilitated the summit. Senior manager 2 provided electronic copies from the Power Point presentations and also gave feedback on the AI intervention detail sheet.

\subsubsection{Findings}

\section{AI intervention detail sheet: Organization 2}

\begin{tabular}{|ll|}
\hline $\begin{array}{l}\text { Title of AI intervention / } \\
\text { Affirmative topic: }\end{array}$ & $\begin{array}{l}\text { Creating [our organization] of the Future } \\
\text { Anticipating and meeting the needs of member } \\
\text { now and in the future. }\end{array}$ \\
$\begin{array}{l}\text { Date of intervention: } \\
\text { Number of employees involved: }\end{array}$ & $\begin{array}{l}\text { August } 2008 \\
110 \text { staff }+20 \text { members }+20 \text { external experts and } \\
\text { business partners }\end{array}$ \\
Number of employees 'impacted': & 1,200 \\
Details of Intervention &
\end{tabular}

Objective:

The focus of the summit or topic of inquiry was how to create our organization of the future by understanding what it will take to fulfill this vision: "[Our region's] most valued organization by 2020 "

In order to achieve this, this organization inquired into ...

- How to live our purpose and vision

- Finding and using our competitive edge

- Ensuring our financial sustainability

\section{Process \& Scheduling:}

The AI-project started in February 2008 involving various project management tasks (formation of a summit committee, organization of the actual summit etc.) Prior to the summit, selected summit participants were trained in the Appreciative Inquiry process and conducted interviews with various staff. These interviews were analyzed and fed into the first phase of the summit. The summit committee was representative of all areas of the business and all levels of employees in terms of hierarchy and also responsible for planning the actual event.

\section{Intended Goals \& Outcomes:}

- Clarity of our organization's purpose and vision

- Understanding our organization's competitive edge

- Increased innovation and creativity

- Greater focus on financial management and accountability

- Understanding of our behaviors that contribute to the member experience

- Greater sense of energy and direction, desire for change and renewal

- Greater sense of collaboration across all functions

○ Our people feeling valued and heard

\section{Interview summaries: Staff (ST1, ST2 \& ST3)}

The findings of interviewing three staff members are presented here in several categories, starting with 'working for this organization prior the intervention'. 
Category: Working for this organization prior the intervention

All three staff members point out the positive culture of the organization before the intervention occurred. ST2 states, "the culture of the [organization] was really quite good. I found it very satisfying working here ... it certainly had a sense of almost family ... [and] I felt that my best interests were being looked at." ST3 argues in a similar way that "the culture of looking after the employees was very good. So I guess the health and safety and employee fulfillment was quite good. I liked that about [our organization]." In addition ST1 points out the member-focused culture stating, "we would try absolutely everything in any way of being able to satisfy members in general terms. Culturally that is our reason for being."

ST1 stressed the financial performance of the organization prior the intervention claiming "we were an organization that did not have a high degree of financial acumen. We did not really focus on the fact that we are a business ... we really were not aware of running a business in a profitable way." This was also due to the strong member focus in trying to satisfy them and give discounts whenever possible arguing that this is where "[we] sort of started being a bit too one sided on the side of the members [and forgot that] we are still here to be a business" (ST1). As a consequence of that "operationally, [this organization] was not running profitably" (ST1).

ST1 also perceives a lack of self confidence in its employees, claiming "we do not trust in ourselves, we do not trust in our own abilities as an organization. We like to bring consultants in to tell us what to do. We had a history of: Let us bring consultants in and they will tell us what to do." Additionally, ST1 perceives that the organization faces difficulties "to do a lot of things at once. We focus on one thing, we fix one thing at a time and then ... move on to a different objective and forget what we were doing before." As a consequence this organization does not "really seem to stick on things for the long term", follow things through and ensure sustainability because "consultants will come and go and you lose that driving force" (ST1).

Category: The two-day intervention

ST1 hesitated to join the summit and was skeptical of the usefulness in doing this, mainly due to two reasons. Firstly, as already expressed above, this new initiative was running a risk of becoming "the flavor of the month, dying very quickly and not having any form of longevity." "We have had a history of commencing new 
initiatives in the past and then not proceeding or not achieving any long lasting changes." Secondly, ST1 was also skeptical of the actual process of the intervention itself, claiming, "ignoring existing issues and problems within an organization was not an appropriate way because unless you acknowledge these issues and do something about those issues they are always going to exist." ST1 agreed to participate only after being persuaded by the manager and the facilitator.

Greatly appreciated by all three staff members was that "for the first time the organization has got a large representation of all levels of the organization and our strategic partners outside" (ST1). "It was really interesting to see all sorts of people" involved in this (ST2). ST1 perceives that "getting a lot of information together, getting a lot of ideas, getting different views, perspectives and getting input from everywhere" as another benefit of holding the summit. As a consequence, there was a lot of engagement and "discussions during those two days about how we can make this place better and some really interesting things came out of it" (ST2).

In reflecting back on the summit itself the three staff members expressed and gave positive feedback in participating, as the following statements will show. "There was a great deal of energy there. I think you kind of get swept up in that positive drive that there was" (ST1). "It was very different ... It was very positive, it was very invigorating actually, it charged me up ... it was inspiring to hear these things and it really, I suppose, made me happy but also made me proud that I was working for this organization ... it was a really positive experience" (ST2). "I guess I enjoyed it ... I think the summit was a great idea as some good things came out of it ... It was very positive" (ST3).

Despite the three staff members reflecting on the summit positively, some negative feedback also came through. ST1 reflects back that the "facilitator really did not answer questions terribly well, particularly questions which were the counterbalance to how we are going to do that, how this is going to happen ... People who were actually questioning things, they were really not given voice and in some cases we were quite shut down." ST3 was unclear about the focus of the summit claiming, "I was a little bit confused as to whom we are addressing. Who we would trying to do this for. Was it for our members, or for our staff ... I do not think that was that clear." 
Category: Afterwards and the outcomes of the summit

After the summit, ST2 remembers coming back "thinking we can do things. The stuff they had been talking about, we can do this. There are some really positive things that we can contribute to this overall plan." In addition participants of the summit were encouraged to "talk about [the summit] ... we were all encouraged to go back and talk and we were all given sort of points to talk to" (ST1). Further on ST1 states, "there was quite a lot of communication out to staff ... and there was a lot of information around it and the key projects that came out if it - there was supposed to be some form of project team coming together for all those objectives." ST1 continues, "that lasted a very short time and really as far as big communication, that happened once."

In focusing on the actual outcomes of the summit, not one of the three staff members could recall all or at least some of them. The only outcome mentioned by each staff member was the business model restructuring (BMR) which happened after the summit and impacted the organization significantly. ST2 claims, "[the restructure] reshuffled the entire organization and in my opinion it turned it right on its head." "It was a major restructure we went through ... we had around about 100 people made redundant out of a workforce of just about 1000. Yes, 10 per cent and a lot of those were mid- to senior-management level ... a lot of people who would have been at the summit left. It was a very unsettling time" (ST1). "Trouble, I experienced a lot of the restructure ... I did not feel comfortable at all because I did not know whether I was going to lose my job either" (ST3).

As a consequence of this major restructure, ST2 suspects that "some of these projects may have fallen by the way side because in the restructure it may not have fitted any more with what the direction in the company was and certain people had left." For example, a person who was heading one project that came out of the summit "was made redundant about 12 months later" (ST2).

A lack of accountability and responsibility for following up on the outcomes and projects of the summit was perceived by ST1. "Someone who is driving it but you have never seen that anyone really was driving it. Whether that is the role of the consultants - I mean we are adults and we should have been taking responsibility ourselves as an organization but I think we fell in a hole of getting distracted by work again" (ST1). 


\section{Category: Now}

ST1 acknowledges that the outcomes of the summit "went very quickly, very quiet and just disappeared" and so "it was just one more thing we did. I think what did happen was we moved on to the next thing and the next thing was the restructure of the organization" (ST1). As a result of that "it just lost momentum and certainly for the last 18 months and it was only 2 years ago, I have not heard the summit mentioned, not seen anything on it and no linkages made to anything" (ST1). ST2 was involved in one follow up project but reflects on the current situation, "I have not been involved, I have not been called on it. It could be still in the background there somewhere but I have not seen visibility of that particular one. I think the BMR became all consuming more or less." Even though ST3 was part of the summit committee, this staff member was not involved in any particular follow up projects of the summit.

As already mentioned earlier, the BMR had a strong impact on the organization and staff overall, causing "uncertainty, a lot of uncertainty" (ST2). ST2 reflects, "it has been a very frustrating and difficult place to work certainly in the last 12 months ... Not to say [the BMR] is not going to be a good thing in the long run just these 12 to 18 months have been really hard." ST1 claims, "if I look at what the summit did and the results of the summit, I see very little has improved in the organization as a result of the summit because it has not been a focus, it has not been a driver and those good ideas that did come up have not been pursued through whatever reason." "I do not believe in the end of the day that we have achieved anything great from it and that is our own failing as an organization but also from the people who ran the summit" (ST2).

As mentioned above, the BMR also had an impact on staff in various ways. ST1 perceives that "staff engagement nowadays is a lot lower and this is driven by the business review ... we survey staff and the engagement had dropped a lot ... we acknowledge that people are unhappy but we have not done a great deal in response to that." As a result ST2 reflects "[in our department] we have had quite a few staff leave in the last 12 months probably." In contrast to ST1 and ST2, ST3 perceives the consequences of the BMR are now settled claiming, "[in my department] I think 
everyone is actually quite happy" and appreciates the culture and "the fact that [the organization] does a lot for its employees."

Despite all the criticism, ST1 highlights that "there is still a member focus - there definitely is still a member focus" and points out the change in the financial focus of the organization. "I would say though however ... the pendulum has swung from being really unfocused and let us just spend money - we have enough money - to really focused." Thus, financial awareness and accountability have increased in the organization.

To conclude, ST1 claims the summit "promised a lot [but] did not really deliver ... it is disappointing to see that happen because there were some good ideas ... so I do not think we have achieved a lot from it. It was a nice couple of days away from the office unfortunately."

\section{Interview summaries: Senior managers (SM1 \& SM2)}

Category: Before the intervention

SM1 experienced working for this organization before the summit as "very positive" but also had to face "lots of challenges" at this time. Acknowledging "there was a culture" SM1 claims that "it was not a dynamic culture." "It is an old traditional organization. So obviously it needs to go through a period of renewal and catching up so to say to 2010 practices" (SM1).

SM2 was part of the project team, organizing the summit and therefore was able to provide insights in being actively involved in the various tasks pre, during and post the summit. The initiative for conducting a summit was driven by one executive member of the organization. "It was [one executive's] idea to actually hold the summit ... and was really the driving force behind it" (SM2). While this executive member was a strong driving force, SM2 perceives that "the other executives did not have buy in. They attended but I think ... they [started to see] some benefits in engaging people [during the actual process and therefore] came on board throughout the summit."

Category: Timing and purpose of the summit 
Both senior managers raised concerns about the timing and the purpose of the actual summit. SM1 reflects that the summit "happened in August and we have done all of our business planning for the year finalized in June ... the strategy was already formed for the year." "The summit would have been way more beneficial if it would have happened in February or March as a preload to business planning" (SM1). According to SM2 the business planning process took the organization about 4 months and "then you bring in [the summit] - and they are going: 'I have just done my plan - I just have my money signed off. What can I change?' That was a really confusing thing for people." "So I think timing was essential in its failure" (SM1).

In asking about the goals of objectives of doing the summit initially, the response of SM1 was "I think this is probably where it goes a little bit fuzzy. I think really we were not clear, [the one executive driving it] was not clear and the executives were not clear from the outset of what they want to achieve from the summit ... as an organization we were not sure why we are doing the summit." SM1 perceives that the summit was "a probably poorly timed event without a purpose."

Category: The two-day intervention

In reflecting on the summit itself, both senior managers gave mainly positive feedback on it. SM1 states, "I think the way the summit was organized and executed was very good. It was executed flawlessly ... the first day we had a combination of staff, business suppliers and members and that was a brilliant day. It was really good to get just the whole community together and go through the discovery and dream phase." The summit "exposed people that worked in different parts of the business, brought them together and they could interact. People actually seemed having really enjoyed doing that" (SM2).

The involvement of stakeholders from the 'whole system' including staff from different management levels, business partners, members, etc. was perceived as another really positive aspect of the summit. "I think for all those people who would not normally be able to participate [in such a workshop] it would have been a very positive experience because they got to have their say. They got to be a part of that collaborative process and it certainly was a step out of their daily life, which is a good thing" (SM1). 
SM2 greatly appreciates the involvement of members in the summit, claiming that having members there "was actually the most positive thing. We should have had more and we should have had members there for two days ... so especially managers all reconnected with why they like working for the organization and how happy our members are ... I think that was very positive and that was probably one of the benefits of the summit" (SM2).

Category: Afterwards

About what happened after the summit, SM1 reflects, "there was no capacity [to implement some of the outcomes of the summit] because all the resources were taken up already in implementing the business plans that happened in business planning." Six project teams were set up to continue work on some of the outcomes of the summit afterwards, but "for everyone else who was outside of these project teams it became irrelevant ... [So] it lost its momentum very quickly ... I would say within six weeks because people just went back to their normal jobs" (SM1). SM2 has a similar perception and claims, "possibly then I do not think we followed up regularly enough. There was a sense people just wanted to move on. Which kind of like missed the whole point."

After the summit "there was a little bit of communication for the months following" (SM1). "There has been some sort of communication ... there was a review. We documented the outcomes and then we communicated the outcomes" (SM2). Still, SM1 perceives that there was "a lack of internal communication planning and organizational development planning, because once this event has happened the whole [concept] was forgotten."

SM1 perceives that the summit was "kind of seen more as an event" with six specific outcomes. Consequently, post the event staff either just wanted to go back to their normal jobs or did not want to actively engage in the projects afterwards or simply have not had the time and resources to contribute to the projects. Due to the lack of follow up on certain outcomes of the summit, SM1 claims that some of the things that were not implemented "got put on the agenda for business planning next time and when we got to business planning next time, people's heads were in a different space again so they had forgotten about it." 
Drawing on the outcomes of the summit, SM2 reflects, "people took the ideas absolutely literally. Those ideas were ideas ... you have to be clear upfront with what are you going to do with the outcomes and tell people right at the onset. So that you do not have any misunderstanding of what the outcomes are going to be." SM2 perceives that some employees might have wanted to see the outcomes of the summit put into action exactly as they were, while some of these ideas were just indicating a direction to where the organization wants to go. Specific follow up projects and intended outcomes would "need a lot more rigorous and checking and testing" (SM2).

\section{Category: Business model restructure}

"One of the things that was brought up [at the summit was that] we actually need to change our business structure in order to be competitive for the future" (SM1). After the summit, the organization went through a business model restructure. SM1 reflects that "the restructure process itself was not a very good change process" and SM2 also perceives that "they handled the restructure really badly over the 18 months." As a consequence "any good bits from the AI summit that should have been brought up in business planning were forgotten ... and people were worried about whether they would have a job or not ... and most of that stuff just got lost because people's heads were in a different space" (SM1).

An interesting detail brought up by SM2 is that the idea for the BMR was something that the executives had already in mind before the summit happened. "I think that was something the executives wanted to do. The CEO said 'I do not want that discussed'. They cannot set the structure at the summit but I think it was almost an enabler because people said the structure does not work. So when people say the structure is not working it shows some acceptance" (SM2). This indicates that the idea for the BMR existed already beforehand but the summit actually contributed to make people more aware of this issue and created commitment to change afterwards.

\section{Category: Now}

SM1 reflects on the situation now arguing, "the culture is having an absolutely crisis based on the restructure that happened last year. So morale is really low and there is lots of turnover happening" (SM1). SM2 claims "we could have got a lot more out of it if we would have been really clear upfront why we are doing this and if all the executives would have been $100 \%$ engaged." "The reality is if I think about how 
much it costs to do the summit I do not think that the intangibles of that summit have actually repaid itself ... [because] if the intervention was about circuit breaking and driving a culture forward to be more self sufficient and motivated and all that kind of stuff, it certainly did not achieve that" (SM1).

Despite this negative feedback, both senior managers also highlight the positives from the summit. SM2 perceives that the summit made it harder to ignore certain issues in this organization. "The restructure, the financial accountability were given a lot more momentum and I think there were certain things that probably would not have got us as far as they have today" (SM2). SM1 would appreciate doing a summit again because a "summit could have a lot potential for this organization specifically in understanding what our members want from us" and now "we would be in a much better point in time to actually do the idea generation and then create action out of it" (SM1).

If this organization was to do a summit again, SM2 points out that the organization should not focus "so much on the event, it should focus more on before and afterwards ... [because] what I now realize is, it is more important on focusing on what is leading up to it and what happens post. It is as important but probably I would say more important."

\section{Interview summaries: General managers (GM1 \& GM2)}

Category: Before the intervention

"I always loved working for [this organization]. It is a good organization. It is a friendly, sort of family orientated organization, sort of family feels sort of place" (GM1). GM2 reflects, "I think the culture was quite good. I think it is a very positive service oriented culture. People care about members and delivering good service."

In contrast, GM1 claims, "this organization was not achieving its potential. It was not performing, as it should operationally." "I would have said a not very efficient organization. It has always been challenging to get things done" (GM1). GM1 and GM2 point out that the structure of the organization was not right, not transparent. "There was something wrong with the structure - it did not work. People were very frustrated with the organization. Decision making, trying to get things done, was very difficult" (GM1). 
"You did not really understand the profitability of the components of each of the businesses. So there was a lack of transparency, I suppose" (GM2). Additionally, "accountability was quite poor in that structure" (GM1). Possibly caused by a difficult structure to work with and a lack of transparency, there was a "lack of financial transparency for each of the functions of [this organization]" as well, which impacted on the actual financial performance. GM1 reflects, "the challenge at the time was, it was not performing financially very well" and "for the first time [this organization] was looking at a quite difficult financial position. Not particularly dangerous but lets just say it was the first time in a long time that it was looking at significant losses" (GM1).

Category: The two-day intervention

GM1 was a bit skeptical doing the summit since it was a significant cost for organization. "It was a very big expenditure that we sunk into as an organization. Being a commercially focused type of person I am always questioning the value of such activities" (GM1). Additionally, GM1 was concerned of what the actual outcomes were going to be.

As part of the preparation activities for the summit, GM1 had to conduct some interviews with staff and find out about positives of this organization. GM1 states, "I found that quite good because I was amazed and surprised of what people thought [this organization] was really good at and where we could improve." These interviews highlighted "the good things that [this organization] did ... it was really good to get that reinforced" (GM1).

GM2 reflects on the summit itself as having "really enjoyed this process, though it was quite effective." "It was an interesting two days" (GM2). "It was very open. That was good. We are quite an open organization and allow people to speak their mind and it was done in that fashion" (GM2). GM1 reflects very positively on the fact the CEO of this organization got up on the stage during the summit and talked about the financial performance and explained that this organization as it is now is underperforming in a lot of areas. GM1 states, "that really had some traction for the staff ... That was one of the big takes out of the whole thing that really resonated with a lot of the people. It was good to see that." 
Both general managers reflected positively on having a diverse and representative group of stakeholders of this organization present at the summit. According to GM1, "the summit in itself was a very positive idea and approach." "I really liked that we actually had our customers there. That is the bit I really liked" (GM1). "[We had] people from all over the organization and people from outside the organization come in, and it was really interesting to hear other people's perspectives on the business and what we do, and how we are perceived" (GM2).

In terms of the outcomes of the summit, GM2 remembers of having a "list of things that were sort of identified as things that we needed to do. The major one of those was looking at the structure of the [organization], the corporate structure. That has led to the business model review, which was rolled out probably 6 months later." Another outcome of the summit was around financial accountability, which "was again picked up as a part of the business model review" (GM2). In asking about other outcomes GM2 reflects, "there were other things that came out but I do not think we got a lot of traction on them at all."

GM2 concludes, "I would say the AI summit we ran was a really good opportunity for our people and our members and others to have an open conversation about [this organization], its past and where they think it should head." "Any organization needs to do a lot of things to engage with their staff and employees - this is a good way to show that you engage with them and take them out of their normal environment" (GM2). "The people who attended felt they were being listened to and the people who did not go were interested to find out what was happening - [there was] good communication afterwards back to the staff about the whole process. So it was good" (GM2).

\section{Category: Afterwards}

After the summit, GM2 was involved in a number of meetings to discuss the outcomes of the summit. As a result of these meetings, project teams "were set up to address the individual components of it" (GM2). Each of the project teams was supported and had "the mandate from the executive team to go and deliver some results and they were supported by cross-functional teams from the various areas had mandate and necessary people to make them work" (GM2). Despite the support of these projects, GM2 recalls, "some of the projects did not sustain. To be honest, in 
something like that I think if you have two or three big successes, that is probably the best you can hope for." Similarly, GM1 claims "I am not too sure that many of [the projects] have got too much traction because the organization has changed so dramatically in the last two years ... I do not think too many of those have sort of really stood the test of time."

One of the big projects that was started after the summit was the business model review, which was a "serious sort of company-wide issue. There was a lot of cost cutting in it as well. We dropped about 130 to 140 staff" (GM2). "There was not one section of the organization that was not impacted by [the BMR]" (GM1). According to GM1, the BMR "was such a dramatic change for the organization that negatively impacted so many people, lots of redundancies, very difficult time for the whole organization, difficult time for everybody in the organization." GM2 claims that the BMR had an impact on the other projects that came out of the summit, pointing out that the resources of any organization are limited and the BMR "was a serious project. So how much attention can you pay to those other initiatives is limited" (GM2). "I think what happens when you restructure ... people's priorities change and then you sort of move on and those other things tend to get a little bit forgotten" (GM2).

\section{Category: Now}

In reflecting two years back on the summit and its outcomes, GM1 claims, "I think it was a good initiative trialed by [this organization]. As an organization that was trying to work out where it needed to go because some of its past things have not worked. I think it was a brave move by the organization to bring in customers, suppliers and key stakeholders into the whole discussion to move it forward." Now, the organization has "certainly a lot more of a commercial focus, a lot more of a cost focus, a lot more clarity. There are positive things; there is no doubt about that. I am very comfortable with what the organization did and why we had to do it" (GM1). GM2 reviews the current situation of the organization quite positively also, pointing out "we are a lot more focused on the business. People are a lot clearer on the strategy of the organization. I think that is fairly clear." When asked about the culture in this organization, GM2 responded, "I think it is a very good culture here ... that sort of service culture and focus on members is very strong." (GM2). 
Despite this positive reflection on the organization at the current stage, GM1 also points out that the BMR also had a negative impact culturally. "There is a little bit of a heart of the organization that is gone and trying to rebuild that and to get some of the passion back in the organization is quite challenging." Additionally, the perception of staff in having a secure employer may have altered as well due to the BMR. GM1 claims "because of that change a lot of people have said "hang on, this is not the place it used to be. It is not as secure as it is used to be so therefore I will assess my view of the organization."

In asking the general managers about doing another summit again, GM2 pointed out "I think there is probably value in doing this sort of inquiry and this sort of summit thing every three or four years. I think it would be worthwhile to revisit that and make sure that we are still continuing that culture of listening to our people and taking positive actions that come out of those sort of summits." GM1 also indicated preferences in doing a summit again but claims "the organization needs to be ready for that and it needs to be in a position where it can act on things that come out of it." "If the organization is structured properly, is in the right space both financially, mentally and capability wise and is then looking for where to go, then I think a summit would be something really good to do. I think the organization was not well positioned to better act on things that came out of the summit because it had all these other issues that were not yet addressed" (GM1).

\subsubsection{Analysis}

In the following, the findings of the seven interviews as reviewed earlier will be discussed together, starting with the category describing the organization before the intervention.

Category: Before the intervention

The interviewees describe working for this organization before the intervention as quite positive and highlight the good culture. ST2 reflects, "the culture of the [organization] was really quite good ... it certainly had a sense of family." "The culture of looking after the employees was very good" (ST3). Similarly, the SM1, GM1 and GM2 express a positive attitude towards working for this organization 
before the intervention. "I always loved working for [this organization]. It is a good organization. It is a friendly, sort of family orientated organization" (GM1).

Having a strong focus on members was perceived as another characteristic of the organization by all interviewees. ST1 points out "we would try absolutely everything in any way of being able to satisfy members in general terms. Culturally, that is our reason for being." "People care about members and delivering good service" (GM2).

While having a strong focus on members and their well-being, the interviewees point out that the organization was lacking a financial focus, accountability and transparency in their operations. ST1 reports, we "did not have a high degree of financial acumen. We did not really focus that we are also a business." GM1 claims, "this organization was not achieving its potential. It was not performing, as it should operationally" and perceived that "there was something wrong with the structure - it did not work."

Category: The two-day intervention

Consistency can also be identified in the way the interviewees experienced the twoday intervention. The involvement of members and external stakeholders in the summit was greatly appreciated. ST2 points out, "it was really interesting to see all sorts of people." "It was really good to get just the whole community together and go through the discovery and dream phase" (SM1). The interviewees reported three main benefits in having a diverse group of internal and external stakeholders involved in the summit.

Firstly, it allowed getting a lot of information, feedback, views and perspectives together, on which basis some good thoughts and ideas could come out and projects formed. Secondly, the summit exposed and reconnected staff to the organization's members. This reminded staff of what this organization is all about and what it can do for the members in the future. SM2 puts it as follows, "especially managers all reconnected with why they like working for the organization and how happy our members are." Lastly, the involvement of staff from lower management levels who would not normally be able to participate in such a workshop, was a positive experience for them because they also got to have their say. SM1 points out, "they got 
to be a part of this collaborative process and it certainly was a step out of their daily life, which is a good thing."

The only negative feedback on the two-day intervention itself addresses an aspect of the facilitation of the event. While the overall, the facilitation was experienced as extremely professional and positive, ST1 reflects critically on the way the facilitators handled more critical questions during the two days, claiming that "people who were actually questioning things - they were really not given voice and in some cases we were quite shut down."

Category: Timing and purpose of the summit

The senior managers raised concerns about the actual timing and purpose of the summit, because business planning had happened just two months earlier and therefore "the strategy was already formed for the year" (SM1). SM2 points out that it took the organization about 4 months to get the plan done and the budgets approved. Consequently, the employees who were involved in the business planning and then participated in the summit did not really know what to do with the outcomes of the summit in a way - "I have just done my plan - I have just had my money signed off. What can I change" (SM1)? According to SM1, this was a confusing aspect for some people. Interestingly, none of the general managers point at this possible conflict in conducting a summit shortly after having completed business planning, even though they were involved in both.

Possibly as a result of the timing aspect, the purpose of the summit was perceived as unclear by SM1, SM2 and ST3 as well. ST3 states, "I was a little bit confused as to whom we are addressing. Who we would be trying to do this for. Was it our members, or for our staff ... I do not think that was clear." SM1 claims, "as an organization we were not sure why we are doing the summit."

Category: Afterwards and the outcomes of the summit

In terms of communication afterwards, SM1 points out that there was "a little bit of communication for the following months." ST1 reflects that employees who participated in the summit were encouraged to talk about it and were given points to talk about. "There was quite a lot of communication out to staff ... and there was a lot of information around it" (ST1). However, ST1 continues "as far as big 
communication, that happened once." Similarly, SM1 perceives that there was "a lack of internal communication planning."

Shortly after the summit, project teams were set up to continue working on outcomes. GM1 reports that each of the project teams was supported and had "the mandate from the executive team to go and deliver some results." Despite this support, "some of the projects did not sustain" (GM2). GM2 claims "I do not think too many of [the projects] have sort of really stood the test of time." SM1 provides a possible explanation for the shortcoming in following up some of the projects claiming, "there was no capacity because all the resources were taken up already in implementing the business plans that happened in business planning."

While six project teams were set up initially to continue working on the outcomes, for everyone else who was not part of these teams, "it became irrelevant ... [so] it lost its momentum very quickly" (SM1). Further on, SM1 claims that people just went back to their normal jobs. Additionally, SM1 perceives that the summit was "kind of seen more as an event" which in turn might have meant that "there was a sense, people just wanted to move on. Which kind of like missed the whole point" (SM2).

Despite the fact that some of the projects have disappeared quietly afterwards, one project that was started was the business model restructure (BMR), which will be discussed next.

\section{Category: Business Model Restructure}

Even though the BMR appears to be a direct outcome of the summit, several interviewees point out that the idea and need to restructure the organization was already 'floating around' before the summit. SM2 says "I think that was something the executives wanted to do. The CEO said I do not want [the structure of the BMR] discussed. They cannot set the structure at the summit." This statement indicates that the CEO had the idea in mind and wanted the organization to go through a restructure in the future. GM1 and SM2 stated that the summit contributed to this discussion, created awareness of this issue, reinforced the need to look at the structure of the organization and created commitment and acceptance to change afterwards.

About 6 months after the summit, the BMR process started, which "reshuffled the entire organization and in my opinion it turned it right on its head" (ST2). According 
to GM2, about 130 to 140 staff were made redundant during the BMR within about 18 months. GM1 reflects that the BMR affected the whole organization, had an impact on a lot of staff and was a very difficult time to for the whole organization to go through.

Due to its significance and the impact this project had on the organization, the other outcomes of the summit were affected negatively. Projects may have fallen away because in the new structure they did not fit any more (ST2), or people were too worried whether they would have a job in the future and likely did not care about the projects that much (SM1) or simply people's priorities change during such a period of change (GM2).

Category: Now and sustainability of outcomes

SM1 points out that the BMR had a negative impact on the culture of the organization, claiming "currently, the culture is having an absolutely crisis." "Morale is really low and there is lots of turnover happening" (SM1). "Staff engagement nowadays is a lot lower" (ST1). GM1 perceives that "there is a little bit of the organization that is gone." In contrast to these statements, GM2 perceives "I think it is a very good culture here ... that sort of service culture and focus on members is very strong."

In terms of the outcomes and projects that were started after the summit, ST1 perceives they "went very quickly, very quiet and just disappeared" and suspects that the "BMR became all consuming more or less" (ST1). Both general managers highlight that the organization now is more financially focused, a lot more cost focused, a lot more focused on the business and "people are a lot clearer on the strategy of the organization" (GM2). ST1 also pointed to this, claiming "the pendulum has swung from being really unfocused and let us just spend money ... to really focused."

Taking the BMR aside, ST1 concludes saying "[the summit] just lost momentum and certainly for the last 18 months, and it was only 2 years ago, I have not heard the summit mentioned, not seen anything on it, no linkages made to anything." ST2, who was involved in one follow up project, has not been involved, not called on or seen visibility of this particular project in the last 12 months. This indicates that this 
project, being one of the follow up projects from the summit, was not sustained nor delivered visible outcomes to the organization. ST2 supports this argument claiming, "I do not believe in the end of the day we have achieved anything great from [the summit]."

\subsubsection{Discussion and summary}

After having reviewed and analyzed the interviews, the question of whether the intervention delivered the outcomes for what it was designed for, will be addressed next.

The first intended goal of the summit was to create clarity of our organization's purpose and vision. With the exception of SM1 and SM2, none of the interviewees even mentioned the words 'purpose' and 'vision' in the interviews. Furthermore, SM1 reflects just on the purpose of summit and not on the actual purpose of the organization. SM2 was involved in the project activities around organizing the summit and therefore was more likely to reflect on this intended goal of the summit. Consequently, SM2 was able to address these topics in more depth explaining, "the executives had done a purpose and vision" (SM2) before the summit "but there was not any substance to it - so what does that mean" (SM2). It is perceived that the summit was intended to create substance and meaning around the organization's purpose and vision. Still, the analysis indicates that neither the summit itself, nor the activities that followed the summit afterwards, may have contributed in raising the overall clarity of the organization's purpose or vision.

The second intended goal is about understanding of our organization's competitive edge. As part of the preparation activities for the summit, GM1 had to conduct several interviews with staff. In doing that GM1 was "amazed and surprised of what people thought [this organization] was really good." The findings of these interviews were used and communicated in the first phase - the discovery phase - of the summit. This indicates that the two-day summit is likely to have enhanced awareness of the organization's strengths and brought up aspects through which this organization can differentiate itself from competitors. However, evidence indicating sustainability of this goal could not be found in any of the seven interviews which is a consistent finding in itself. 
Increased innovation and creativity was the third goal of the intervention. ST1 perceives that the summit got input from the various stakeholders and put a lot of information and ideas together. As a result of the discussion over these two days "some really interesting things came out of it" (ST2). Several interviewees reflected on two ideas that came out of the summit to make this organization more accessible by utilizing technology better and critically reviewing the touch points of the organization with its members. This indicates that the summit fostered creative thinking in making the organization a better place and as stated above brought up some "really interesting things" (ST2). ST2 reflects coming back and "thinking we can do things. The stuff they had been talking about, we can do this. There are some really positive things that we can contribute to this overall plan."

Even though creative and innovative ideas might have come out of the summit, SM1 explains that there was not much capacity left because all the resources were taken up already in implementing the business plans. After all, how does an organization benefit from having creative people and innovative ideas if there is no capacity and resources left to take advantage of the ideas? In other words, the good ideas might not be valuable at all if an organization is not capable in exploiting them. While the summit appears to have increased innovation and creativity and delivered specific ideas, the interviewees indicate that in following up these ideas, the organization failed to do so.

Greater focus on financial management and accountability was another intended goal and outcome of the intervention. GM1 and GM2 claim that the summit made the need to look at the organization's structure, improve the organization's focus on financial management and accountability quite apparent. Whether the BMR was a direct outcome of the summit or not, the summit contributed in creating commitment to follow up on the restructure of the organization. Likely as a result of the BMR, GM1 states, that the organization now has "certainly a lot more of a commercial focus, a lot more of a cost focus, ... a lot more clarity. There are positive things; there is no doubt about that." GM2 points out that the organization "had a really strong year in a pretty tough environment and a lot stronger focus on the business." This evidence suggests that the summit raised awareness of financial management and accountability issues initially, while the restructure of the business, which happened afterwards, addressed 
and strengthened this issue again and according to GM2 resulted in improved financial performance.

Understanding of our behaviors that contribute to the member experience represents another goal of the intervention. Due to the involvement of 20 members on the first day of the summit, employees had the chance to interact with them. SM2 reflects back, "the members that attended - think it was about 20 members - was actually the most positive thing. We should have had more and we should have had members there for two days." The opportunity to engage with members was greatly appreciated since it also allowed staff to hear what members think and know about this organization. For ST1 it was interesting to see how little the members know about what this organization actually does, which shows "despite the fact that we market it all the time, ... put out all this information but the message is not getting out there."

This example illustrates how the involvement of members in the summit gave the employees the opportunity to see the organization from a member's perspective and enabled them to identify areas where the organization can do better in improving member interaction and member service. Based on this evidence, it is likely that the summit contributed in understanding the members better but also recognized areas in which the organizations has shortcomings in delivering good member experiences.

The last goal of the summit was to have a greater sense of energy and direction, desire for change and renewal with the two sub-goals of having a greater sense of $\underline{\text { collaboration across all functions and our people feeling valued and heard. }}$

Certain aspects of this goal tie into some goals discussed earlier and therefore will not be discussed in great detail again. The goal of achieving a greater sense of energy and direction ties into the first goal of achieving clarity on the organization's purpose and vision. As discussed earlier, the summit made the deficiencies of the organization's structure, financial management and accountability apparent to the employees and is likely to have caused commitment to change and renewal.

Only the two senior managers use the words collaborative/collaboration once in their interviews and refer to the AI process itself but do not specify whether the AI summit had an impact on enhancing collaboration across functions or not. Due to the absence of specific evidence, any form of argumentation would be speculative. 
Drawing on the goal of giving employees a feeling of being valued and heard, GM2 states "[the summit] is a good way to show that you engage with [staff] and take them out of their normal environment." "The people who attended felt they were being listened to" (GM2). Additionally, all interviewees reflect collectively very positively on being part of the summit and enjoyed having the opportunity to speak up and interact with the various participants. This indicates that the summit is likely to have contributed to making people feel valued and heard.

To conclude, the two-day summit itself appears to have contributed to achieving most of the intended goals and outcomes. Even though, little evidence could be identified that these goals and outcomes were sustained over the last two years. Consistency in the findings could be identified in having a greater focus on financial management and accountability currently as well, while the achievement of the other intended goals and outcomes is more difficult to judge. Still, a tendency to choose a lack of achievement is likely to be argued for by the interviewees. 


\subsection{Organization 3}

The third organization involved in this research directly employs about 50 staff and is a non-profit organization in its nature. In addition, about 200 volunteers support the organization in delivering its service.

Seven interviews with various staff and external stakeholders of this organization were conducted. The interviewees were three external stakeholders to the organization, two staff members, one senior manager and the former CEO of the organization. The interviewed former CEO left the organization about 8 weeks before the interview was conducted. Since this interviewee left the organization very recently, this was not considered to be problematic in involving this former employee in this research. The senior manager and the CEO are both addressed with the term senior manager to ensure confidentiality among staff in the organization.

At the time the intervention happened, the organization consisted of about 50 employees, who all participated in the summit. Since the intervention also included about 80 external 'stakeholders' (supporters, donors, volunteers etc.) of the organization, three interviews with members of this stakeholder group were conducted. All the interviews were recorded and lasted 40 minutes in average. The following table presents further details on the interviewees.

Table 5: Details of Interviewees - Organization 3

\begin{tabular}{|c|c|c|c|}
\hline Details of Interviewees: Organization 3 & 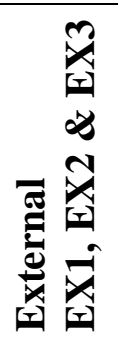 & 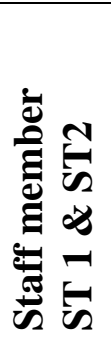 & 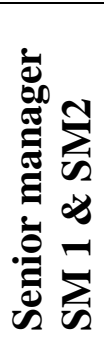 \\
\hline $\begin{array}{l}\text { Average number of years engaging with } \\
\text { organization }\end{array}$ & 5.7 & - & - \\
\hline Average number of years in organization & - & 5 & 5.5 \\
\hline Average number of years in position & - & 2.5 & 6 \\
\hline
\end{tabular}

Source: Compiled from data collected

The AI intervention detail sheet for this organization was compiled from project summary documentation prepared by the consultant, who was in charge and 
facilitated the summit. This consultant is an experienced senior consultant with several years of work experience in designing and facilitating AI interventions.

\subsubsection{Findings}

\section{AI intervention detail sheet: Organization 3}

\section{Title of AI intervention /}

Affirmative topic:

Date of intervention:

Number of employees involved:

Number of employees 'impacted':

Details of Intervention
Propelling [our organization] into an innovative and sustainable future

February 2008

130 participants ( 35 staff +25 community partners +25 volunteers +45 external experts, board members, academics in the field)

35 staff +200 volunteers

\section{Objective:}

The objective of the summit was to bring together the stakeholders and employees of the organization in order to identify and define the goals and objectives of the organization. These goals and objectives are mentioned below.

\section{Process \& Scheduling:}

Planning for the summit began in September 2007. A steering committee (representative selection of employees from the whole organization) was set up to guide the design of the summit. A planning committee was made responsible for the implementation of the day-to-day project tasks.

Research champions were advised to lead teams on researching and interviewing local and global experts in various areas, which are of interest to the organization. Each team prepared a paper on their research findings that would comprise some of the prereading for summit participants. Posters and displays that visually represented their findings were used during the summit.

The first step in designing the summit involved training of steering and planning committee in the AI methodology. Following this, the actual design of the summit was developed.

Two weeks before the summit, a pre-Summit pack was sent to participants. The purpose of the pack was to brief participants with enough key information about this organization.

\section{Intended Goals \& Outcomes:}

- Create a strategic plan, including a review of the Mission, a 2020 Vision, 2012 Goals and initiatives

- Achieve a 'one [our organization]' focus

- Support the leadership transition

- Create organizational confidence and skill in asking critical questions

- Affirmation of [our] values

\section{Interview summaries: Staff (ST1 \& ST2)}

Category: Working for this organization prior to the intervention 
ST1 describes the organization as a small, sort of family feel organization in which "everybody knew what everybody was doing and they were all working on similar things" (ST1). Both staff members reflected positively on the culture, which "has always been really great and positive" (ST1). ST2 states "everybody would pitch in to do whatever needed to be done ... people would drop tools and help."

Work was organized and progressed in a more reactive rather than proactive way. ST1 remembers, "there were lots of things coming in and being done straight away. There were not many processes in place ... we were just reacting to opportunities that came up rather than having something be able to say." ST2 explains, this style of working and leading is also due to the founder and leader of the organization, who "did not micro manage people." Additionally, "there was not a lot of reporting back to [the founder] about what we were doing. It was a very loose form of leading” (ST2).

In terms of communication ST1 points out, "communications has always been easy. We have a very flat-structured organization. Even though we have a CEO and manager, the communication is never sort of elemental to what role you play and what position you have."

Category: The two-day intervention

ST1 experienced the two-day summit as a process that was "obviously quite fun but also brought everyone together in an inclusive process. It generated excitement and enthusiasm." ST2 remembers, "I found it quite hard actually. I found it quite confronting because it was emotional. There were a few tears." From an organizational perspective, ST2 criticizes that the summit was a massive outgoing expense but also recognizes the benefits claiming, "I definitely saw the value in it" (ST2).

ST1 appreciated that the conference "had such a variety of different people there. So you had staff, ex-staff, our young people, you had funders, professionals in the same sector as us. When I think back, the highlight was there were so many people there, everyone was equal and everyone had their say." As a result of this ST1 felt "more connected to the organization and the people involved ... felt more excited and focused about where we are going as an organization." 
Both staff members greatly appreciated the inclusiveness of many different stakeholders in the summit, which allowed establishing and strengthening the networks within the community of this organization. ST2 reflects, during the summit "we connected with various individuals" because "the activities meant that we had to work with a lot of different people" (ST1).

Category: Afterwards and the outcomes of the summit

Post summit, both staff members experienced a lack of follow through and keeping up the momentum that was created in these two days. ST2 claims, "we lost that momentum. We lost a massive opportunity as an organization."

As mentioned earlier, the summit was experienced as a very emotional and interactive process, where participants were "caught up in the moment of having this great conference with so many people" (ST1). As a result of this, the expectations of the participants on continuing the momentum and involvement of the community in the future were high. ST2 reflects that the expectations created during the summit were not met afterwards. Two possible reasons for this lack in follow through can be identified.

Post summit, working groups (project groups) were set up to continue working on five projects that came out of the summit. Participants of the summit could sign up for these working groups and continue their involvement. ST1, who was involved in one working group, claims that the "objectives [of these projects] were a bit difficult to relate to." The organization "potentially should have worked on these a little bit more afterwards" (ST1) in order to create more clarity around these projects. Additionally, ST1 also experienced a lack of structure in the working group ST1 was involved in. As a result of a weak structure and unclear direction, this working group fell apart "within a month. Yes. I think we had a really light and fluffy group" (ST1). ST2 concludes, the working groups "had poor outcome data."

While the working groups faced severe difficulties, another reason for not keeping up the momentum was perceived in having poor communication afterwards. ST2 points out, "we were going to have regular updates and feedback and none of that has actually happened." "I would have maintained more communication externally. I believe that would have made a difference in the way we are as an organization now" 
(ST2). "We invested a lot of money in attending, running and doing AI and invested time in connecting with people" (ST2). After this ST2 concludes, "it would have benefited the organization to keep these people engaged and on board financially."

Despite these negative aspects in following up, ST1 reflects that the summit "definitely assisted us in raising more money" from the donors who attended the conference. In addition, the summit "helped us to get us back on track. I think we might have been drifting as an organization" (ST1). One outcome of the intervention was the strategic plan including its goals and objectives, which was developed within the first few months after the summit. ST1 points out, this strategic plan has given the organization direction and "is something we can refer to and use in our work."

\section{Category: Now}

The strategic plan and goals helped the organization to align its tasks towards these goals and were also "really helpful to induct new staff" (ST1). Furthermore, ST1 perceives that these goals themselves are one of the few tangible outcomes that are left from the summit.

The business plan, which was finished by the end of 2009, also caused changes within the organization. ST1 reflects, the business plan "is a big step that we had to make and we had a restructure based on that business plan." As a result of this restructure, roles within the organization were made redundant and new roles created. In addition to the restructure, two programs (two services this organization offers) were stopped, and this caused further disruption within the organization (ST2). Lastly, this organization has grown significantly again since the summit (ST1). Potentially caused by all these changes, only " 12 people are left in the organization that participated in the summit" (ST2). As a result, "we do not talk about the summit [any more]. People know that we have our goals and strategy but they do not know the history of where that has come from or how that has started" (ST2).

ST2 concludes, "AI was an opportunity for us as an organization to involve and work with all stakeholders but we did not take up this opportunity fully, which I think is a shame. And because it is not embedded in the organization, it has got lost." 


\section{Interview summary: External stakeholder (EX1)}

The findings of the three external stakeholders are presented separately, since their background, level of involvement, etc. is quite diverse and a combined presentation of the findings might be confusing for the reader. In the following, the findings of the external stakeholder 1 are presented.

EX1 started engaging with this organization a few years before the intervention "as a user of their services but had no real contact with the organization" (EX1). After the summit, EX1 became an ongoing volunteer and more engaged in the organization.

EX1 reflects, the summit itself "was incredibly intense ... it was pretty much a dream experience for me at that point - quite amazing." The summit invited different stakeholder groups, which were encouraged to share their experiences in being involved in this organization. This aspect potentially "created a much bigger sense of interconnectedness within the organization ... there was a lot more goodwill for the organization" (EX1).

Additionally, the summit encouraged participants to think big and dream without limitations. "It all felt very dreamy ... [but] there was not any thought about practicalities" (EX1). "So you had these wonderful two days where you were dreaming, coming up with all these great things and you had people really sucked up and then the implementation of all these crazy things proved not to be so practical" (EX1).

On the second day of the summit, participants were invited to sign up for the working groups. EX1 joined one of these groups claiming, "when you are at the summit that seems like the best thing ever." While people "were really committed at the conference" and willing to continue their involvement, one of the downfalls was that "a month or two months later, you have lost the hype, you have lost the insanity, the craziness and you do not care as much" (EX1). Consequently, meeting these high expectations and fulfilling the commitments turned out to be difficult to achieve post summit.

After the summit "you had this wonderful afterglow effect" and now it appears that the summit "promised a whole lot and achieved very little" (EX1). EX1 was involved in the working group for about half a year and contributed on average 4 hours per 
week to this project. Despite this extensive involvement, EX1 perceives that the working group did not achieve very much in the end. "It was casual talk and very little action" (EX1). EX1 concludes, "it is one thing to do the dream, design, destiny phases and then it is another thing to follow it up and make sure it does work and make sure the positive outcomes are delivered upon because I feel in this case they have not been."

\section{Interview summary: External stakeholder (EX2)}

EX2 - a senior manager with extensive work experience - started to support this organization about 5 years ago. EX2 reflects, supporting this organization "seemed to be a really good cause and it was an easy way of helping and you could see that both the money and [other things] that were donated were going directly to the kids." Additionally, EX2 "was very impressed by the fact that there did not seem to be political issues or having a bureaucratic structure" and you could see that the "money and the efforts were going directly into the programs" (EX2).

The conference was "really an issue of making all the participants aware of the significant [...] issues that are confronting young people" (EX2). EX2 reflects that the conference was well organized, professionally run and that it was inspirational to see what this organization does and potentially can do in the future. "It identified for me, I am sure for the other participants as well, the need, the awareness of a social obligation, that we should all undertake" (EX2). After all, the conference "committed me to make a bigger contribution ... I walked out of the conference even more determined to provide financial and physical help" (EX2).

Post summit, this interviewee was invited to engage actively with the organization and contribute financial expertise to the organization. More specifically, a finance subcommittee was formed in which EX2 is a member of. In being part of this committee EX2 provides expertise on the organization's financial results, improving operational performance and cutting out bureaucracy.

This interviewee's involvement in the finance committee is still ongoing, meeting once every month. EX2 specifically appreciates that this organization is able to deliver its services in a non-bureaucratic, non-political and cost-effective way. EX2 
concludes, "I am just there to help and not necessarily to change things because I could see the organization was running quite well anyway."

\section{Interview summary: External stakeholder (EX3)}

EX3 started engaging with the organization as a volunteer in 2004. In being involved in this organization EX3 reflects "I really got a sense of how good the organization was and how effectively they engaged the volunteers and young people." Further on EX3 explains, "what was keeping me there was my relationships with the staff and the other volunteers. There was a real sense of doing good in the community but also sharing that experience with other people." The tasks EX3 fulfilled as a volunteer were diverse starting from presenting information to schools and universities, speaking to organizations etc.

The summit "was probably one of the best things I have ever been involved in. It was just an incredibly powerful and amazing process to go through" (EX3). The involvement of supporters, donors and other stakeholders in this process was greatly appreciated. EX3 says, "it was quite a nice and lovely experience to be able to talk to people who were donating money or resources or whatever to the organization and share my experience and compare that with their experience."

After the summit EX3 joined one of the working groups with the task to develop strategies for "engaging with the broader community and capturing that community feel" (EX3). This working group engaged for about 8 weeks but unfortunately, "that flopped. I mean we put our recommendations forward and nothing happened with it" (EX3). Another critical reflection addresses the involvement of external stakeholders after the summit. EX3 explains that the summit created this "really high energy and really high expectations of the results" among the participants. External supporters wanted to help and continue their involvement but the systems and processes to facilitate this were not in place so "quite a few people felt quite upset and excluded" (EX3) post summit. It was "almost like the conference never happened for some supporters" (EX3).

EX3 continued working as a volunteer but was also elected as a member of the board of directors. EX3 explains "me being made a director was probably a result of the 
experience that I had at the summit." The summit gave EX3 the opportunity to get to know the board members, establish networks and just collaborate with them.

In focusing on the organization now, EX3 claims, it is "still very dynamic, but they are acting more focused [towards the mission and goals] than before". "The 2012 mission is still really strong. All the staff know it and they know what all the goals are that they are aiming for" (EX3). Even so EX3 perceives, "the atmosphere that we created over those few days dissolved."

\section{Interview summaries: Staff (SM1 \& SM2)}

Category: Before the intervention

The time before the intervention was characterized by a strong growth of the organization, during which it was driven by a really strong vision and a strong founder. SM2 states, "we had this really strong sense of purpose and really strong sense of where we wanted to go." Further attributes used by the senior managers to describe the organization are "entrepreneurial spirit" (SM1), "very passionate and committed" (SM2), "really dynamic and a sense of collegiality" (SM2), "a lot of collaboration" (SM2) and "flexible organization and flexible workplace culture" (SM2).

Both senior managers, however, point out that the organization was also characterized "by a bit of a lack of formal structure and process" (SM2) and not having "much planning and processes" (SM1) in place. SM1 reflects, "one day we were focusing on this, the next day we were focusing on something else." As a result, "the organization has been pulled in many different directions and trying to be all things together. The leadership team had difficulties in prioritizing of all choices you could do, what were the ones to invest in because they did not have a strategy" (SM1).

Category: The intervention

SM2 remembers the summit saying "they were full on, really intense, it was a very exciting event ... I thoroughly enjoyed myself." SM1 reflects, the summit "created this sense we are all in this together." "There was this great sense of everybody that was in the room felt a really strong connection to each other" (SM1). Additionally, the summit "was sort of a high point experience in [this organization's] history" (SM1) 
and caused "lots of emotions" (SM1) among the participants. The involvement of young people and a diverse group of external stakeholders was perceived as another great benefit in holding the summit since it exposed the organization and enhanced collaboration. SM1 remembers, "the organization was totally inward looking the whole time and AI broke it open and made it look outward."

In terms of outcomes of the summit, SM2 reports that "there was a lot of energy, a lot of ideas ... there was some greater clarity and there were some new collaborations formed." Additionally, SM2 claims "there were a lot of expectations that needed to be met." The summit created high expectations among the participants and stakeholders in following up the summit. One of the outcomes "was expectations around increased stakeholder involvement and certainly one of the outcomes was increased community around [this organization] ... I think not everyone's expectations on that were met" (SM2).

SM2 perceives that AI makes a lot of things appear to be possible, opens things up, collects the best of people's experiences and allows participants to think big. As a result of this we "ended up with a plan that was a little bit big for us" (SM2). "There was not necessarily sufficient either process within the summit itself or as follow up to actually then really take another critical look at what had been agreed on coming out of the summit" (SM2). SM2 concludes that the plan that came out of the summit "was too ambitious" and there were no "decisions made about scaling that back" (SM2).

Category: Afterwards

In what happened after the summit, "we had not prepared for ... [because] for every high point in somebody's existence or an organizational life there is a corresponding low point" (SM1). The summit was an "incredibly intense emotional high point and we did not prepare anyone for the time afterwards" (SM1). SM1 suspects that employees might have experienced coming back to work as a shock because "at that point nothing had changed yet ... It is suddenly this back to reality." SM1 explains that employees expected that the organization would be different right away. "People wanted to change it overnight" (SM1). 
Another critical aspect both senior managers highlight are the high expectations in following up the summit. SM2 explains the organization was not prepared for delivering on some of these expectations and "that is where we started to run into problems." For example the external stakeholders expected an ongoing high-level contact to the organization, being involved in all sorts of things, etc. but in turn the organization was not able to follow up. SM2 explains, AI consumed a lot of resources and added extra stuff to the organization and afterwards "we were all exhausted." Consequently, there was not much energy left to allocate even more resources towards the projects.

In terms of the working groups that were formed in the summit, SM1 reflects, "some of them worked really well, some others did not." SM2 explains that some groups faced difficulties because they "were left on their own to determine how they would take parts of the plan forward." Furthermore, there was a "lack of follow up, especially the working group that I was part of and not being clear of what decisions need to be made about how that working group would take its work forward" (SM2).

SM2 concludes, "the follow up could have been more guided and more structured." "It felt like we came out with some really concrete objectives from the AI summit that we then were not able to let go of. We could have perhaps chunked off huge pieces and said 'that might be a thing for 2015' rather than kind of having to do it all in this period" (SM2). The expectations, the promises and the overall 'plan' from the summit appear to be too ambitious to be handled appropriately with the available resources and so "that story dissolved or faded in a way that I was surprised by" (SM2).

\section{Category: Now}

SM1 perceives that the summit delivered the outcomes it was initially designed for claiming, "we went in there very clearly stipulated on what we want to get out from these two days and it delivered every one of those." Even so, SM2 argues "would I say the summit is living, does AI live on in our organization? My feeling is no, it does not" because "there are not really symbols in the organization that remind us about that AI was part of our journey" (SM2).

As an outcome of the summit, the organization now has a strategic plan, "which we are working towards, [which] guides our work and is still a living document" (SM2). 
SM1 notes, "everyone in the organization can cite that strategic plan. We have complete buy in."

Referring to the external stakeholders who participated in the summit, SM2 points out that there were some participants "whose commitment and involvement with [this organization] has deepened as a result of the summit." However, SM2 also reflects "there were some people who were disappointed with the lack of follow up, whose expectations were quite high in terms of follow up and they were not met."

SM2 concludes, AI "is not an end destination, it is a process and in my organization I think we experienced it as an end destination rather than a process ... For lots of people and certainly in my mind, AI became the summit rather than the summit being part of the AI process."

\subsubsection{Analysis}

In the following the findings of the seven interviews conducted will be discussed together, starting with the category describing the organization before the intervention.

Category: Before the intervention

The external stakeholders' involvement in the organization before the intervention appears limited. EX1 and EX3 were users of the services while EX2 engaged with the organization as a supporter. Due to the minor involvement of these stakeholders, they did not focus much on the time before the summit in their interviews.

ST1 describes the organization as a small and sort of family feel organization that went through a period of strong growth. The influence of the founder, a very visionary person, on the organization was high and because of this person the organization "had this really strong sense of purpose and really strong sense of where [...] to go" (SM2). Furthermore, the organization is characterized as having passionate and committed employees, an entrepreneurial spirit, a lot of collaboration among the employees and good communications (SM1 \& SM2).

Additionally, the interviewees reflect that the organization did not do much planning and did not have many processes in place (ST1). The organization progressed in a 
more reactive rather than proactive fashion. SM2 claims, the organization was characterized "by a bit of a lack of formal structure and process."

Category: The intervention

The interviewees showed great appreciation of being involved in this two-day event. EX1 reflects, the summit created "a much bigger sense of interconnectedness within the organization" and "a sense we are all in this together" (SM1). ST1 \& ST2 highlight the benefits of including the different stakeholders in the summit since this allowed the organization to connect with various individuals and strengthen relationships. The summit was experienced as quite fun (ST1), emotional (ST2 \& SM1), exciting (ST1 \& SM2), powerful (EX3), intense (SM2) and amazing (EX1 \& EX3). The conference raised EX3's awareness of the significant issues that are confronting young people and therefore identified the need of a "social obligation that we should undertake" (EX2).

At the end of the summit, participants had high expectations for achieving the things that have been discussed at the summit. Additionally, the external stakeholders expected to be more involved and engaged in the future activities of the organization. SM2 perceived that the organization was not ready and did not have the resources to follow up on all of these expectations and so the organization "ended up with a plan that was a little bit big for us."

Category: Afterwards

As noted, the organization ran into difficulties in delivering on what the summit had promised. SM1 points out that the summit participants had high expectations on what would happen post summit while the organization was not prepared for this period. The systems and processes that allow the organization to process all the ideas, promises and expectations that came out of the summit were not existent. Consequently, SM2 reflects "the follow up could have been more guided and more structured.”

The working groups that were formed to continue working on the outcomes of the summit also faced difficulties. SM1 reflects "some of [these groups] worked really well, some did not." The interviewees involved in working groups report of a lack of structure (ST1), having an unclear goal and a light and fluffy group (ST1), difficult 
objectives to relate to (ST1), a lack of following up (SM2) and not being clear about how to take the work forward (SM2). EX1 perceives that their working group did not achieve very much in the end. "It was casual talk and very little action" (EX1). Similarly, the working group of EX3 flopped. EX3 reflects, "we put our recommendations forward and nothing happened with it."

Despite facing difficulties in following up the outcomes of the summit, the intervention also led to some positive developments afterwards. ST1 points out, the summit "definitely assisted us in raising more money" from the donors who attended the event. EX2, whose involvement increased as a result of the summit, reports that the summit raised awareness among the donors, sponsors and other stakeholders. Furthermore, the summit made the significance of this issue apparent and encouraged the stakeholders to make a bigger contribution in the future. As mentioned above, EX2's involvement in this organization increased. On the other hand, ST2 reports that expectations of some other external stakeholders were not met and they therefore drifted away from the organization. EX2 claims, "it would have benefited the organization to keep these people more engaged and on board financially."

ST1 states that the summit supported the organization to realign itself and "helped us to get back on track". ST1 continues, "I think we might have been drifting as an organization." SM1 explains that the summit supported the organization in going through a restructure and taking some uncomfortable decisions because the employees saw the necessity to do that. Even though it was not easy, the organization managed to go through this difficult time of change.

\section{Category: Now}

The organization as it is today has changed significantly since the summit in 2008. As mentioned earlier, the organization restructured, dropped some programs that were not performing, and also experienced staff turnover.

In asking one interviewee what is left of the summit, ST2 points out that only 12 people are left in this organization now who also attended the summit. Furthermore ST2 says, "if we would just look at AI and the office now, most people would not have a clue of what we are talking about." SM2 has a similar perception, claiming that $\mathrm{AI}$ is not alive any more and "there are not really symbols in the organization that 
remind us that AI was part of our journey." "We do not talk about the summit [any more]" (ST2) and "the atmosphere that we created over those few days dissolved" (EX3).

The strategic plan including the organization's mission and goals were created as a result of the summit and are still very strong. SM2 claims, this plan is something the organization works towards and is a document employees refer to. EX3 concludes, "the 2012 mission is still really strong. All the staff know it and they know what all the goals are they are aiming for." SM1 says, "everyone in the organization can cite that strategic plan. We have complete buy in."

\subsubsection{Discussion and summary}

Based on the findings of the interviews, the question, whether the intervention delivered the outcomes for what it was designed for, are addressed next. In doing so, the intended goals and outcomes of the intervention detail sheet are compared with the findings of the interviews as presented earlier.

The first intended goal and outcome of the intervention was to create a strategic plan, including a review of the mission, a 2020 Vision, 2012 goals and initiatives. The findings indicate that the summit assisted the organization to develop these outcomes. SM1 reflects, "We have got a strategy, we have got a new mission statement, we have got a 2020 vision, we have got goals, we have got an implementation plan, we have got all that out of the summit." Additionally, SM1 continues saying, "everybody walked out feeling like it was their plan, they wrote it, so we had a lot of buy in." It has to be noted that the summit did not deliver these outcomes by the end of the second day of the intervention, the goals and the strategy were further developed, refined and communicated afterwards. ST2 states, "[the strategy and goals] were discussed for many months and then they were devised and I saw that as a sort of the main outcome [of the summit]."

In elaborating on sustainability of the mission, goals and strategy that was developed, SM2 states, "we have got a strategic plan, which we are working towards, which guides our work and is still a living document." EX3 explains, that the mission is still really strong and staff know the goals they are aiming for. Everyone in the organization can cite that strategic plan (SM1). "The strategic plan is always 
something you can refer to and use in our work since then" (ST1). These quotes indicate that the strategy, mission and goals that came out of the summit and were further developed afterwards, are two years post the summit still really strong, in the employees' minds and most importantly used and applied by the employees.

The second intended goal and outcome of the summit was to achieve a 'one [our organization]' focus. The summit brought a diverse group of the organization's stakeholders together and sought their collaboration in making this organization work at its best (SM2). SM1 reflects, "the organization was totally inward looking the whole time and AI broke it open and made it look outwards." SM1 continues, "everybody that was in the room felt a strong connection to each other" and "it created this sense we are all in this together" (SM1). EX1 remembers, the summit "created a much bigger sense of interconnectedness with the organization." Similarly, ST1 felt "a lot more connected to the organization" after the summit.

While these quotes strongly argue that the summit itself contributed to achieving this goal in the short-term, the findings on the long-term perspective of this goal appear less positive. ST2 reflects, "AI was an opportunity for us as an organization to involve and work with all stakeholders but we did not take up this opportunity fully which I think is a shame and because it is not embedded in the organization, it has got lost." ST2 continues, the summit "was an amazing opportunity to have all the stakeholders [continuously involved] but we did not follow through." SM2 says, "would I say the summit is living, does AI live on in our organization, my feeling is no, it does not." SM1 explains that the organization was not prepared to follow up the high expectations of external stakeholders. The necessary systems and processes to continue ongoing high-level contact with these stakeholders were not in place and therefore this community feeling could not be sustained. SM2 concludes, the community feeling which was created at the summit "has not had continuous momentum and conversation and not being communicated externally. It is like it fizzled out which I think is a shame."

To support the leadership transition was another goal of the summit. Two aspects of the organization's past have to be considered in discussing this goal. Firstly, the organization went through a period of strong growth and developed a working culture of everyone knows everything and is able to implement everything (SM1). This 
resulted in, as SM2 explains, a period which was "characterized by a bit of lack of formal structure and process." Secondly, the organization was formed by a visionary and "much loved character" (SM1). Before the summit, this founder informed the employees about stepping down from the organization and moving on internationally. SM1 explains, "everyone in the organization was very worried" whether it would be still the same without the founder leading. This goal therefore was created to highlight these two issues and help the organization to address them appropriately.

SM1 reflects, "the summit created evidence that the organization at its core was going to be the same." One outcome of the summit was the employees "framed [the founder's] leaving into something suddenly possible and positive" (SM1). Therefore, the employees saw that there is a future without the founder leading the organization. SM1 says, the summit "was the very symbolic handover [that] this organization is no longer about [the founder]. This organization is about its mission and being here for young people."

In addition, the summit supported the leadership transition in creating awareness among the employees that the organization is not performing at its best in order to fulfill its mission. The summit created a strong sense of direction and where the organization should go and focus at (SM2). Following the summit, employees saw the need to change in terms of structure and leadership in order to be better positioned to deliver on its mission. Even though it was a difficult and unsettling change process, employees accepted it. SM1 reflects, "we closed programs, we let staff go ... [but] they understood because they have been at the AI, they totally endorsed the strategy." Consequently, these findings indicate that the summit supported the organization's leadership transition.

To create organizational confidence and skill in asking critical questions was another goal of the summit. In reviewing this intervention, four aspects could be identified that address short and long-term aspects of this goal.

Firstly, the summit exposed the organization to its external stakeholders. SM1 reflects this turned the organization inside out, claiming, "the organization was totally inward looking the whole time and AI broke it open and made it look outward." Thus, the 
organization was willing to ask and include its stakeholders in determining its future and also be open to criticism.

Another aspect ties into the goal presented previously addressing the issue that the organization had to change in terms of its leadership structure and go through a process of restructuring itself. The summit made it obvious to the employees that the structure as it was, was not supporting the organization in achieving its mission. As already mentioned, the summit facilitated this change process since it had the buy-in from most of its employees.

In terms of sustainability of this goal, it is difficult to argue conclusively in either way. In terms of communication, the organization struggled to keep the external stakeholders informed and actively involved in what the organization does. The channels of communication to this group of people were shut down in many cases. ST2 argues, "I would have engaged them more. It would have benefited the organization to keep these people engaged and on board." At this stage, the organization is not involving this group of external people as much as expected after the summit. Consequently, it appears that the organizational confidence in listening, asking and involving these people has dispersed.

On the other hand, this organization now has systems and processes in place in order to critically reflect on its own performance and draw its conclusions from it. SM1 points out that measurement systems are now in place and are used to decide on whether certain programs are worth investing further resources or not. SM1 reflects, "we closed programs. Like programs that were not performing, not delivering results." In this case the organization asks these critical questions about the fruitfulness of certain programs and bases its decisions and future actions around the answers to these questions.

The last intended goal and outcome of the summit was the affirmation of [our] values. The summit reflected the values of this organization greatly. SM1 points out, it "it matched [us]. It brought out the best in [us]." Particularly the involvement of young people in the summit was highly regarded since this organization is about young people and being there for young people. While the summit allowed the employees to reflect back on their own and the values of the organization, ST2 perceives that the 
values "are still stable over time ... and my values align with the organization's values."

To conclude, the AI intervention in this organization appears to have delivered on most of the intended goals and outcomes. In asking SM1 whether the intervention delivered the outcomes it was initially designed for, SM1 claims "Yes. I think yes without a doubt. We went in there very clear stipulated what we want to get out from these two days and it delivered everyone of those." Building on the earlier discussion, this statement of SM1 can be confirmed. Even so, the findings also reflect on the difficulties the organization faced post the summit. A severe issue for the organization was to cope with the high expectations in keeping the external stakeholders informed and engaged post the summit and to continue the community feeling that was created at the summit. 


\section{Chapter 5: Discussion}

In looking back to the cases, the findings show that there were simultaneously positive, as well as negative developments in these three cases. Hence, this discussion focuses primarily on the issues and aspects occurring in the three AI interventions that appear to have impacted on the success. In order to do so, the intended goals and outcomes - reason for doing AI - need to be reviewed and discussed first. Subsequently, a discussion about aspects impacting on achieving these intended goals and outcomes will follow.

\subsection{The purpose of AI - AI for what?}

AI is used for a purpose; more specifically AI is used to achieve certain outcomes that the organization wants to achieve and it perceives is worth achieving. The three organizations expressed their reason for doing AI through their affirmative topics and the intended goals and outcomes. Still, in looking back on the three interventions, it appears that there are several types and categories of goals and outcomes that can be achieved through an AI intervention, as shown in the following:

- The goal and outcome stated by the affirmative topics

- Intended goals and outcomes

- Provocative propositions

- Short-term goals and outcomes

- Long-term goals and outcomes

Even though goals and outcomes of the AI interventions studied may fit into two or more types simultaneously, these types and categories are not the same. In some AI interventions, the organizations did not seem to distinguish between these categories and ensure the alignment of the goals and outcomes across the categories.

The affirmative topic of the second case, for example, focuses exclusively on meeting the demands of the organization's members in the short- and long-term. Yet, four out of the eight intended goals and outcomes have little or no direct connection to the member focus of the affirmative topic. Furthermore, these eight intended goals and 
outcomes address a diverse range of topics and are unlikely to align and support each other. In addition, the six provocative propositions (six projects that were started as a result of the summit) are only in alignment to some of the intended goals and outcomes. All together, in this case 15 goals and outcomes needed to be considered in the intervention.

In contrast, the first organization had only four intended goals and outcomes and all of them had a long-term focus. In addition, the four goals appear to be in alignment to each other and towards the affirmative topic. Post the summit no additional projects were started. Lastly, the third case had five intended goals and outcomes (two shortterm, 3 long-term) and an affirmative topic, which was to a great extent aligned with the intended goals and outcomes. The following table provides an overview of the goals and outcomes across the three organizations.

Table 6: Goals and Outcomes from the AI interventions

\begin{tabular}{|c|c|c|c|}
\hline & $\overrightarrow{0}$ & $\underset{\omega}{0}$ & $\underset{b}{\infty}$ \\
\hline Affirmative topic & 1 & 1 & 1 \\
\hline Intended goals and outcomes & 4 & 8 & 5 \\
\hline $\begin{array}{l}\text { Provocative propositions / Projects that } \\
\text { were started post the summit }\end{array}$ & 0 & 6 & 5 \\
\hline Goals and outcomes are aligned & YES & NO & YES \\
\hline Focus: Long-term vs. $\underline{\text { Short-term }}$ & $\mathrm{L}$ & L\&S & L\&S \\
\hline Total Goals and Outcomes & 5 & 15 & 11 \\
\hline
\end{tabular}

Source: Compiled by researcher

Drawing on this table and the discussion of the three cases, it appears that the interventions with fewer goals and a stronger alignment between these goals were more successful in the short as well as the long-term. While Org1 successfully achieved all its goals and outcomes, Org2 struggled to keep projects alive and was not able to achieve all of its eight intended goals and outcomes. Even though Org3 achieved most of its intended goals and outcomes, the performance and results of the projects started post the summit was overall poor. 
The literature presents the affirmative topics as the inquiry's focus, which are developed before the summit (Whitney, Cooperrider, Trosten-Bloom \& Kaplin, 2005). Whitney et al. (2005) suggest selecting between three and five topics that meet the criteria of being affirmative, desirable, genuine and will take you where you want to go. Provocative propositions are developed as part of the third phase of the summit picturing the organization in the future when "the positive core is fully effective in all of its strategies, processes, systems, decisions and collaborations" (Cooperrider et al., 2008 , p. 162). While the process of developing provocative propositions and affirmative topics are well explained, it appears the literature does not address in detail how to consider the intended goals and outcomes (the reasons for doing AI) and ensure their alignment. If an organization decides to deploy AI to achieve ' $A$ ' but the summit delivers ' $\mathrm{B}$ ', their objectives were likely not met. While ' $\mathrm{B}$ ' might be even more beneficial for the organization, the actual reason for doing AI was not achieved. AI advocates might argue that the intended goals and outcomes are implied through the affirmative topics and the provocative propositions are a result of the affirmative topic (Cooperrider et al., 2008). This appears to be the case for the first and third organization, yet the goals and outcomes of the second organization were not fully aligned. Faure support this argument claiming, "it is surprising how many management teams either fail to think through clearly what their own expectations are or fail to communicate these expectations ... we must ensure that senior management thinks through these issues in a rigorous fashion" (2006, p. 27).

As this discussion shows, to be clear about the reasons why an organization wants to do an AI intervention and what it wants to achieve from it are of critical importance. Thus, to clearly distinguish between these different types of goals and outcomes appears to be beneficial in enhancing discussion about them and ensuring their alignment.

\subsection{The build up to the summit}

\subsubsection{Timing of the summit}

Whitney \& Cooperrider state that a "summit works best when there is a need to accelerate the process of change" $(1998$, p. 17). While this statement links the timing of the summit to the need for change of an organization, the three cases show that 
other internal and external factors may also have contributed or hindered the organizations in going through an AI intervention. Due to the existence of these factors, the 'right' timing of an intervention can be of great importance to make an intervention successful.

Regional manager 2 (Org1) pointed out that the timing of the summit coincided with a number of other things going on. The economy was flourishing, lots of investments were being made and the organization had performed well financially. RM1 reflects, by having the summit at that point in time it was "almost the perfect storm in a positive way." Consequently, the summit was potentially reinforced by these positive internal and external developments.

The second case shows a scenario in which the timing might have been wrong. The organization went through the annual business planning processes shortly before the summit happened. The interviewees of this organization pointed out that there was not much capacity left to also implement all the things that came out of the summit, as resources were already taken up implementing the business plans. In addition, the organization had planned to restructure the business and post the summit actually went through a major restructuring process. As a result, the intervention and some follow up projects did not get a lot of traction.

In the third case several interviewees pointed out that the timing was good because the summit created awareness around certain aspects within the organization. This organization had expanded and grown over the previous years considerably and offered services that were not always aligned to the organization's purpose. The summit supported the organization in realigning itself to its mission and helped in identifying and making necessary changes afterwards.

These external and internal factors strongly to have had a strong impact on the interventions. The timing of the second intervention was problematic and simply by postponing the intervention by six to nine months, the two problematic factors might have become irrelevant or even disappeared. GM1 (Org2) summarizes that an organization needs to be in the right space to go through an AI intervention. An organization needs to be ready for the summit and ready for what comes afterwards. 


\subsubsection{Preparation for the actual event}

Despite most interviewees not referring much to this aspect, the amount of work needed to organize a summit of 130 to 320 participants is enormous. In all three cases, it took the organizations between six months and one year to go through the necessary preparation tasks for the summits. Faure suspects that "change efforts that work do so because their leaders have paid greater attention to creating the right conditions for change" (2006, p. 22). In addition to creating the 'right' conditions for change, the summit itself needs to be prepared as well. This involves tasks like inviting employees and external stakeholders, organizing a venue, providing necessary pre-information for participants, organizing the tasks of the summit itself, defining the purpose of the summit including its goals and outcomes, training a core team of employees in the AI methodology, etc. Due to all of these necessary efforts, Faure highlights that running an AI summit with anything more than 40 to 50 participants takes a great deal of planning, preparation, and structure (2006). Given the investments needed, it becomes highly desirable, if not essential for the summit to deliver successes.

Note: The three AI interventions were designed and facilitated by very experienced senior consultants or groups of senior consultants with various years of work experience in field of change management, AI etc. The total costs of these interventions (involves preparation activities and the summit itself) were between NZD 400,000 and over a million NZD (costs do not include the time-investment-costs by the employees).

While the required workload as well as the financial investment needed for a summit of over 100 participants is significant, the interviewees recognized the huge effort the organization was taking. In many cases the interviewees were impressed to see that the organization would spend that much money on this, which in turn showed the organization's commitment and commitment from management to this intervention.

\subsubsection{Customization}

The literature on AI does not provide an explicit outlined method or even a recipe that an organization should follow (Salopek, 2006). It is still the task of managers, consultants and facilitators to be aware of local characteristics, internal and external variables etc. and consider these appropriately in designing an AI initiative. While the 
main structure (discover, dream, design and destiny) of a summit remains set, the consultants and facilitators are still required to 'fill' these phases with activities and tasks that are appropriate and suit the organizations.

As the three cases show, not only the summit but also the whole intervention needs to be planned rigorously in order to make it successful. For example, an organization should probably have a 'plan' in place, explaining what should happen post the summit. Org1 started to engage all its employees once a week to carry on and integrate the 'thought' of AI into the organization right after the summit. Since most employees of Org1 work in the branch network with fixed opening hours, these meetings are scheduled outside the normal business hours in order to allow all employees to participate in these meetings. This is one good example of how a customized approach of using AI in an organizational setting can be successful.

The argument here is that in any organization there are known and unknown variables that can have an impact on the success of an intervention. Therefore it is essential for AI consultants and practitioners to consider these variables and customize the AI intervention accordingly in preparing a summit because, as Bushe states, "AI is still affected by all the traditional change variables ... [and $\mathrm{AI}$ ] does not create change without a whole lot of the wisdom of 'traditional OD' applied competently" (2007, p. $35)$.

\subsection{The summit}

\subsubsection{Appreciation of diversity among participants}

Throughout the three cases, the interviewees consistently enjoyed having a diverse group of people present at the summit. The perception of SM1 (Org3) that the summit "created this sense that we are all in this together" seems to be shared among all interviewees. The involvement of external stakeholders in the summit specifically was highly appreciated by the employees of Org2 and Org3. The summit represented a great opportunity to reconnect with these external partners. On many occasions, the employees were reminded of why this organization exists, what it is good at and how satisfied external people are with this organization. Several reasons can be identified, 
which in combination led to the appreciation of having a diverse group of people participating in the summit.

Firstly, the summit represented an opportunity get in touch with various employees, stakeholders, partners, members, customers etc with whom someone usually would not have the chance to interact. Due to the engaging activities as part of the summit, this process enabled employees to establish and strengthen networks with all of these various participants. Whitney \& Cooperrider support this argument claiming "the summit builds and renews relationships across the organization and among employees, customers, and vendors" (1998, p. 19).

In having a diverse and representative group of people from the 'whole system' participating, the summit created this 'one organization feeling'. As SM1 (Org3) stated the summit participants 'realized' that they are all part of this organization. As a result of this SM1 (Org3) reflects "there was this great sense that everybody that was in the room felt a really strong connection to each other."

Lastly, in having the whole system represented and contributing, the summit created a strong momentum for action. The argument that "the summit creates confidence and commitment in the organization by liberating the ideas and opinions of all participants" (Whitney \& Cooperrider, 1998, p. 19) can be confirmed. The interviewees particularly appreciated that everyone had a 'say' and everyone could contribute to make things happen. ST2 (Org2) remembers after the summit "thinking we can do things. The stuff they had been talking about, we can do this. There are some really positive things that we can contribute to this overall plan."

\subsubsection{Summit results in excitement, hopes and wishes for the future}

In reflecting on the findings, Faure's statement that the positive focus of "the AI process naturally results in many positive emotions, such as pride, satisfaction, hope, amusement and gratitude" (2006, p. 25) can also be confirmed.

The interviewees reflected very positively on being part of such an intervention. They commonly used words like 'fun', 'great', 'amazing', 'exciting', 'energizing', 'motivating', 'making you feel special' to describe this event. Even employees, who 
were skeptical about the usefulness of summit beforehand, actually saw the value in doing it and enjoyed being part of it (ST1-Org2 \& GM1-Org3).

In trying to find an answer for why a summit causes these positive experiences among the participants, the research performed by Whitney \& Trosten-Bloom (2003) is interesting. They performed an inquiry into why the summit of an AI intervention works and discovered what they call the six freedoms / six conditions. It is argued that AI works particularly well because "it unleashes all of the six freedoms over a course of just one complete 4-D cycle" (Whitney \& Trosten-Bloom, 2003, p. 239). These six freedoms are (1) the freedom to be known in a relationship, (2) the freedom to be heard, (3) to dream in community, (4) to choose to contribute, (5) to act with support and (6) the freedom to be positive. The findings on how the interviewees experienced the summit tend to correlate with these six freedoms positively. Thus, findings of this research support the conclusions made by Whitney \& Trosten-Bloom.

\subsection{Post summit}

\subsubsection{Back to work!}

As discussed above, the summit represents an amazing and remarkable event for the participants i.e. energy levels are high, wishes and hopes were raised and bold expectations are created. Across the three cases, employees experienced the time right after the summit as 'coming back to reality / back to normality' since nothing had changed so far. Three issues were identified that made it 'difficult' for participants of the summit to come back to work.

The summit creates this picture in the mind of people of 'what could be' and how the 'perfect' world would look like. Even though employees understand that these are dreams, there might still be an element of disappointment to realize that actually nothing of this has become reality so far. It is still exactly the same organization as it was before the summit.

Another quite obvious aspect is that the normal work of employees is still there and needs to be done. While the summit already increases the workload an organization has to perform, involving about $10 \%$ of the total workforce in a two-day event increases the work that needs to be done after the summit again. As SM2 (Org3) 
stated, there were heaps of e-mails to be answered and simply the work that built up over the last few days needed to be done because, as ST2 (Org2) explained, work is always going to be there for you and never disappears. Consequently, for a lot of participants it was a matter of jumping straight back into their jobs and getting on with the work.

The 'frozen chicken metaphor' illustrates the third difficulty for employees coming back to work. A consultant interviewed in a previous research project pinpointed the issue with this suitable metaphor explaining: What happens if you take one frozen chicken out of the freezer, cook it and put it back into the freezer with the other frozen chickens? Either it freezes again or goes bad. In inviting an employee to take part in a summit and putting them back into their 'old' departments with co-workers who did not participate in the summit, this particular employee might feel uncomfortable and probably even isolated in not having shared these great experiences. This resistance can be seen in these examples. The branch managers (Org1) experienced some resistance from subordinates to adapt to this new style of engaging them in the weekly WoW meetings. BM5 (Org1) pointed out, it was a matter of getting employees slowly on board. ST2 (Org2) also, explained that existing staff did not know what this event was all about and therefore were not really interested in it.

These three issues show why employees might experience the time after the summit as difficult and challenging. Thus, to prepare the summit participants for this 'aftershock' in coming back to work appears to be useful, i.e. be upfront with employees and realistic about what will happen post the summit. Furthermore, employees are likely to need support to avoid reverting back to 'normal' and freezing again.

\subsubsection{The case of misperceived expectations}

As discussed previously, the summit encourages participants 'to go big', let go of any limiting thoughts and be positive, with little time spent on thinking about the practicalities and feasibilities (resources, time, money etc.) of the 'things' that participants come up with during the summit. While top management may understand that some of these ideas are not realistically achievable right away or in the near future, other stakeholders might be disappointed to see that little may change quickly, e.g. a year down the track. In the second case, GM2 (org2) was pleased to see that the 
summit helped to deliver one or two specific outcomes. In contrast to that, ST2 (org2) was disappointed with what happened afterwards claiming, "I do not believe in the end of the day we have achieved anything great from it."

Similarly, EX1 (org3) was very disappointed to see that the things the summit promised were not delivered. For example, this interviewee expected to receive regular updates from the organization or become more involved in the organization's activities and be able to contribute. While SM1 and SM2 (org3) are aware of these 'shortcomings' and issues, they explain that the organization was not able to follow up on all of these things and had to prioritize projects. In addition, the organization was not prepared for the amount of communication that would have been required to keep all the stakeholders informed and engaged.

These two examples show that the participant's hopes, wishes, dreams and expectations that the summit created were, most of the time, not met afterwards. While top management understands that the organization's resources are limited and change needs to be planned and cannot happen right away, participants with less involvement in management activities might not see that. Hence they are likely to be disappointed to see that the organization is basically still the same, with change happening slowly and in the 'old fashioned' or traditional manner. Even though it contradicts with the philosophy of AI, it appears to be important to be realistic and upfront with participants about what they each can expect from the summit (in terms of following up the 'promises' and provocative propositions).

\subsubsection{Provocative propositions and the follow up projects}

The provocative propositions formulated during the summit are, "presenting clear, compelling pictures of how things will be when the positive core is fully effective" (Cooperrider et al., 2008, p. 162). In the last stage of the summit, destiny, these propositions are 'put into action' by establishing projects and project teams around them in order to ensure that these propositions are achieved. When summarizing the success of the projects that were started in org2 and org3, a suitable statement might be 'AI hits reality'.

Reflecting on org3, the interviewees reported several difficulties (already high workload, unclear structure, objective not specified fully enough, project not 
realistically achievable) in continuing to work on these projects. Even if the projects then delivered outcomes and ideas, EX1 (org3) explained that these results were sometimes then not used and not put into action by the management. Similarly, org2 faced almost the same difficulties. As a result in both organizations many interviewees indicated that there is very little left from these projects or AI itself.

So what might be the issues with these propositions and projects? One reason, as SM2 (Org3) pointed out, was that the propositions were too big and too ambitious. The organization should have "chunked off huge pieces" and postponed some projects to a later date (SM2-Org3). The summits invited participants to think big, without limitations and just forget about 'all' constraints. As a result of this, the organizations ended up with a plan that was too big to achieve in one step. An interesting suggestion to tackle this issue is put forward by Ncube \& Wasburn (2008). They added a distill phase, after the design stage in order to "refine and prioritize initiatives" (2008, p. 17) that come out of the previous phase. In doing so, the big plan can be scaled down to a feasible workload-level.

As already mentioned in the 'back to work' sections, employees got distracted by the day-to-day work after returning from the summit and therefore, might not put that much effort into pursuing the summit projects. Faure states in reviewing an AI initiative she facilitated, "the weaker projects ran into the day-to-day business realities and began slipping down the agenda" (Faure, 2006, p. 28). This appears to have happened in some instances within Org2 and Org3.

In contrast to Org2 and Org3, the first case presents an AI intervention without having specific follow-up projects post the summit. Instead the organization exclusively focused on rolling out WoW to the whole organization and achieving the intended long-term goals and objectives. Considering the success of WoW and the intended and unintended positive achievements it brought, the question whether an AI initiative needs to have specific follow up projects arises. Drawing on the research performed by Bushe \& Kassam (2005) they come to the conclusion that out of 20 reviewed cases, only seven were transformational. Interestingly, in six out of these seven transformational cases the organization did not use action teams or project teams post the summit. Instead, Bushe (2007) explains, "they adopted an 'improvisational approach' to the action phase." (2007, p. 35). Similarly, Org1 did not put its focus on 
achieving specific projects outcomes and instead rolled out the AI mindset throughout the organization.

\subsection{Sustainability}

In order to discuss the aspect of sustainability in a purposeful manner, the question regarding the 'sustainability of what' needs to be addressed first. As stated in the beginning of the discussion chapter, each organization used AI for a specific purpose and expected to achieve certain outcomes and goals with it. Hence, in this discussion, sustainability of AI is defined as 'ongoing (over)-achievement of these intended goals and outcomes'.

Reflecting on the intended goals and outcomes across the three cases, some of them are focused on achieving the short-term objectives. As an example, one intended goal of Org3 was to 'support the leadership transition.' Arguably, a discussion about sustainability of a short-term focused goal becomes redundant unless the organization reverts back to its former approach. Consequently, the discussion of sustainability is more appropriate for goals and objectives with a clear long-term orientation.

In comparing the intended goals and outcomes across the three cases, the findings show that only the long-term goals of Org1 could be achieved. One major difference between Org1 and Org2 \& Org3 is that post the summit, Org1 started to have weekly mini AI engagement sessions in which current and relevant business topics were covered. Over the period of 4 years since the summit, these meetings are still continuing. In contrast to this, $\operatorname{Org} 2 \&$ Org3 set up various project groups to continue working on the outcomes of the summit. As the findings show, these project groups tended to fall over and had to a great extent poor performance as far as outcomes go. Consequently, the only 'reminder' that AI is/was part of the organization's history is extinguished and AI was forgotten. In both cases (Org2 \& Org3) the interviewees clearly pointed out that AI now ( 2 years / 2.5 years after the summit) is not 'alive' any more. In contrast to that, organization one celebrates the birthdays of WoW and still engages employees in the weekly WoW meetings.

The findings show that all three organizations initially faced difficulties and obstacles in 'sustaining' AI. The branch managers (Org1) reported resistance from staff to adapt to this new form of engaging employees and did not get full buy in right away. In the 
case of CCM2 (Org1), the roll-out of WoW even failed in the first place. As discussed above, the second and third organization also faced severe difficulties in getting traction and results out of the projects and as time passed these projects disappeared quietly. So, all organizations had to overcome resistance, obstacles etc., but only Org1 managed to deliver results in the long-term. This raises the question, what is the difference between project groups (Org2 \& Org3) and weekly engagement meetings (Org1) in creating and sustaining long-term results through AI? This question will be addressed next.

\subsubsection{Involvement of the whole organization/community}

Organization one started to engage the 'whole organization' in the weekly AI meetings after the summit happened. It was the task of the branch managers (Org1) to roll out WoW to their subordinates and get them involved. As a result of doing this, about 2,500 employees got engaged and in touch with WoW. In strong contrast to that, Org2 and Org3 'only' had the project groups with a very limited number of employees involved in place. SM1 (org2) reflects, "for everyone else who was outside of these project teams it became irrelevant" or perhaps it never became relevant at all!

Interestingly, to 'exclude' employees in following up the summit contradicts with AI's philosophy. AI advocates claim that, AI 'works' best when a large and diverse group of people is engaged around a shared theme and therefore able to contribute. To put project groups in place, which limit the number of people who can contribute is not in alignment with AI. The weekly WoW meetings of Org1, on the other hand, were designed to engage almost all of the employees from the organization.

\subsubsection{Focus on achieving long-term results vs. focus on projects}

Another difference between Org1 and Org2 \& Org3 can be recognized in having a different focus in what these organizations did past the summit. Org1 used the weekly WoW sessions to engage 'all' employees in order to achieve the intended long-term goals and outcomes collectively. In this case, the focus of why doing AI did not change. The summit simply represented a major event that was used to get enough traction for WoW but after that the weekly sessions reinforced the focus on achieving long-term results. 
Reflecting on the findings of Org2 and Org3, it appears that the initial focus of these organizations changed from achieving their intended goals and outcomes towards achieving specific projects that were created after the summit. It almost appears that having these projects in place distracted the organizations from achieving the initial long-term goals and outcomes. In addition, a strong focus on projects also assumes that there is an end to these projects. Arguably, if an organization has long-term goals, that indicate a direction on where the organization should go and focus on, replacing these goals during the summit with specific projects might not be beneficial in achieving these long-term goals; particularly if the organization does not have followup processes in place. In addition, it does not help to embed AI into how the organization progresses.

\subsubsection{An argument for follow-up AI engagement meetings}

Building on the discussion above, it appears that regular (weekly, fortnightly or at least monthly) employee engagement meetings involving preferably the whole organization might be needed in order to continue the thought, positivity, enthusiasm, etc. that was created during the summit and achieve the intended long-term goals and outcomes after all.

The team of consultants, who developed WoW, argue in one of their reflection documents, "it typically takes 18-24 months for new organizational practices and mindsets to become completely integrated and fully sustainable" (Consultant). In reflection on the interviews with Org1, WoW represents a remarkable milestone in the organization's history, which "transformed [this organization's] culture" (Org1-RM2) and is still alive and celebrated. Faure supports these arguments claiming that an organization should "allow enough time if the goal is transformational change" (2006, p. 29).

To conclude, this discussion on sustainability points out that an organization may need to have 'reminders' (e.g. weekly employee engagement meetings) in place that reinforce the message that was sent out to employees at the summit. This seems particularly important, if the goal is to achieve long-term goals through an AI intervention, because "AI does not result in a revolution in change in and of itself" (Bushe \& Kassam, 2005, p. 177). 


\subsection{Consistency across different stakeholders of the organization}

Reflecting on the summit itself, the interviewees offered consistently positive views of the event. As discussed earlier, independent from their roles, working background, responsibilities etc. the interviewees collectively enjoyed being part of such an event (Org1, Org2 \& Org3).

Inconsistencies across the different stakeholders and levels of the organization could, though, be found in how the interviewees experienced the time after the summit (Org2 \& Org3). In particular, the expectations on following-up the summit differed between the groups of interviewees. While senior management and higher realized early on that not everything that came out of the summit can be achieved afterwards, other stakeholders were disappointed to see little/no changes happening post the summit.

Likely as a result of having different expectations on following-up the summit, senior and top-level management were more satisfied with what AI 'achieved' and therefore, tended to categorize the intervention as successful. In contrast to that, interviewees of other stakeholders groups (ST1-Org2, ST2-Org2, EX1-Org3 \& ST2-Org3) showed their disappointment and claim that from their perspective the summit was not successful. Again, awareness of such differences in perceptions will need to be recognized and addressed following an AI summit.

\subsection{The 'essence' of AI}

In processing all the information the interviewees provided, three statements/quotes particularly stood out. These three quotes appear to be representative concluding statements, to which other interviewees, from the same organization, are likely to agree with. However, they also indicate a common understanding following an AI initiative that can occur independent of project success.

Quote 1 - organization one: "It is not 'what do we do with WoW' but 'how do we use WoW to help us do the things we are trying to do in the business, ", (RM2-org1)

Quote 2 - organization two: "Reflecting on the Summit brought an analogy to my mind - a marriage vs. a wedding 
Much of the time when people get married, the focus is on the wedding day, who to invite, what to wear, food, drinks, rings, speeches, cars etc. What really should be focused on before the wedding is building a strong bond, relationships and common goals and after the wedding, building a strong marriage, which requires continual work and reinforcement.

The summit is the beginning of a marriage not an event. The organization needs to understand this, commit to the future outcomes and at the summit we have the opportunity to co-create the future. Most of the weaknesses with [our organization's] summit lie in what happened before the summit and after the summit. In future dialogues and deliberations we need to ensure that as much effort goes into this as the planning of the actual day” (Source: Reflection document written by SM2-Org2).

Quote 3 - organization three: "[AI] is not an end destination, it is a process and in my organization I think we experienced it as an end destination rather than a process ... for lots of people and certainly in my mind, AI became the summit rather than the summit being part of the AI process" (SM2-Org3)

Drawing on these three concluding statements, the perception of the interviewees of what AI represents and stands for within organizations can be different; AI as a task, something an organization has to do vs. AI as a tool, which supports an organization to do things.

When the organizations started to plan and roll out the AI initiatives, AI might have been perceived as a task; something the employees had to do because the topmanagement decided to do it. In rolling out WoW to the whole organization (Org1), some employees did not see the reason for doing this, showed resistance and thought it will be only temporary and disappear. Consequently, during this time, employees participated merely because they had to and so it was just a task; something the employees had to do. Only after time passed by, did employees start to see the value in doing WoW, what they could get out of it, enjoyed being part of these meetings and finally contributed. As a result of these changes in perception, WoW was not perceived a task any more; it became an accepted organization process that was used 
by the employees to talk about the business. WoW finally became a tool, as RM2 (Org1) states, which supports the organization do the things it wants to do.

In contrast, this transformation in the way employees perceive AI never happened in Org2 and Org3. SM2 (Org3) clearly points out that AI is more than just an event that should happen once. AI is not just an end destination called the summit itself. As recognized by this employee, the organization, unfortunately, perceived AI as a one off event; an event the organization had to go through. Therefore, AI might have become not much more than a task; a task that you do once and then move on to the next thing.

The statement of SM2 (Org2) shows a very similar story highlighting the aspect that the summit itself enjoys a lot attention while the 'time' before and after the summit is likely to be more important that the event itself. Similar to a wedding, the summit 'only' shows a visible expression of commitment but in order to make a marriage/AI work, it is ultimately the things that happen afterwards.

\subsection{Summary}

In this chapter the findings from interviewing 23 employees and stakeholders are compared across the three participating organizations. These findings align to a great extent with the literature on AI. Particularly, the literature describing the summit itself is in line with the findings. Still, as this discussion shows, the summit represents only a part of the whole picture and therefore, new aspects, adding on to this picture, were identified and highlighted through within and across case analysis. 


\section{Chapter 6: Conclusion}

The purpose of this research was to evaluate AI interventions in order to

- Show the benefits of using AI and its capabilities,

- Identify weaknesses and shortcomings that might inform future AI interventions and

- Provide an approach for how evaluation of AI interventions could be done.

The interviewees across the three organizations provided sufficient information, on which basis these research objectives could be met. Some of the strengths of AI that could be identified in the discussion chapter were AI's ability to engage a large and diverse group of people, enhance collaboration among them and create commitment to achieve shared goals.

In contrast, a main weakness and shortcoming of the AI philosophy may be its 'narrow' focus on the summit itself. In this research, it is argued that the time after the summit is at least as important as everything that comes before. Yet, little attention is given to this critical time afterwards. The sections 'results of this study' and 'managerial implications' cover this aspect in more detail again.

Lastly, this research presents an approach for how AI interventions can be evaluated. Interesting feedback and results were captured with this approach on which decision makers can draw on and inform their decisions.

\subsection{Results of this study}

Among many findings and results that this research unveils, three of them stood out over others and are worth mentioning again. These three aspects are covered in the following subsections and implications for future AI interventions made.

\subsubsection{Ensure diversity of participants at the summit}

As shown in the discussion chapter, having a diverse group of people involved in the summit was greatly appreciated across the three cases. The interviewees consistently enjoyed having the opportunity to talk and engage with various people they would not normally have the chance to in their usual work environment. A consultant 
interviewed in my previous research project, explained that, the summit participants should be representative and involve stakeholders of the whole system an organization operates in. Arguably, the organization's stakeholders can vary significantly. Some examples of stakeholders the three organizations invited to their summits were employees, customers, members, suppliers, academics, experts in the field and supporters.

Three benefits in involving a diverse group of stakeholders to the summit were identified. As mentioned above, the interviewees were given the opportunity to get in touch and engage with various participants, allowing them to establish and strengthen networks with them. Secondly, in having all these people in the same room and engaging in several activities, the summit created this 'one organization feeling' in which participants realized that they are all part of this organization and establish a 'connection to each other'. Lastly and likely reinforced by the first two benefits, in having all these stakeholders involved, the summit created commitment and momentum for action.

\subsubsection{The summit is just the beginning}

"This is not the end. It is not even the beginning of the end.

But it is, perhaps, the end of the beginning."

(Winston S. Churchill in Collins \& Porras, 1994, p. 201)

As Churchill purposefully used this quote to emphasize the dangers of declaring victory too soon after a won battle, consultants and practitioners as well, should bear this quote in mind and be aware that the summit is only the beginning of something and not the end. The summit represents an enabler to achieve goals and objectives and therefore, should not be the goal itself. Just by having a great summit experience, an organization is in no position to celebrate and relax. As shown in the discussion chapter, the time after the summit appears to be even more crucial for achieving the organization's goals and objectives. Thus, practitioners, consultants, management, as well as the summit participants need to fully understand the implications of this quote in order to create traction and commitment for the time after the summit. 
In reviewing the literature, great emphasis is put on the planning and the execution of AI summits. Less information and discussion on what should happen post summit is available. Recognizing the large diversity of an organization's processes, communications, cultures, structures, etc. it appears to be very difficult to provide a more prescriptive approach and concept for the time after the summit. However, 'avoiding' this topic entirely may lead to the misperception that the summit is the AI intervention and not only a part of it. The next section includes further details on the three organizations post the summit.

\subsubsection{Feed AI into organizational processes rather than projects}

To better understand the need for a strong focus on the time after the summit, the approaches chosen by the three organizations are considered specifically. Org1 used weekly employee engagement meetings to integrate AI into their organization. In these meetings, Org1 engaged all employees (in groups between 5 to 30 people) by applying the AI philosophy, with respect to relevant business topics. Org2 and Org3, on the other hand, put specific projects in place that were a direct result of the summit.

In comparing these two approaches, the findings clearly show that the approach applied by Org1 successfully integrated the AI's philosophy into the organization's processes and ways of doing things. In contrast, the projects of Org2 and Org3 tended to fall over quickly and the outcomes were to a great extent unsatisfactory. Several reasons can be identified that might have contributed to the success / caused the failure of these approaches. A major difference between these two approaches is that Org1 involved the 'whole' organization in this engagement process, while the project approach chosen by Org2 and Org3 involved only a limited number of employees.

On the one hand, Org1 put the meetings on the weekly agenda of their employees and there was no 'debate' about it. Thus, employees simply did not have a 'choice' whether to participate in these AI engagement meetings or not.

For Org2 and Org3, on the other hand, everyone who was not part of the projects, AI became or remained irrelevant. Putting a strong emphasis on projects is likely to create the perception amongst employees that there will be an end to these projects and the AI philosophy. The findings support such an argument and illustrate how employees 'went back to normal' after the projects either failed or were terminated 
(Org2 \& Org3). Lastly, the employees working on these projects were often 'distracted' by their day-to-day work. While the summit itself already increased their workload, for the employees working on the projects it was then even one more thing they had to do. Therefore, they might not have pursued the success of these projects as fully as of adequate time and attention had been given.

This discussion strongly suggests that organizations need to emphasize the time after an AI summit. It is during this time when energy and enthusiasm for the summit's agreed outcomes will be highest and change may be most achievable. As outlined above, integrating AI's philosophy into organization's processes (e.g. through weekly engagement meetings) is likely to be more successful than relying on specific projects (that are started as an outcome of the summit). This approach also has the benefit of bringing AI to others in the organization who were not at the summit.

\subsection{Managerial implications}

Instead of putting forward specific managerial implications that address only a few aspects of the intervention, this research clearly points out that it is the 'whole' intervention (before the summit, the summit itself and the time afterwards) that affects AI's sustainability. Thus, a proposed approach on 'how to do an AI intervention' is put forward. This proposed approach is informed by the literature as well as the findings of this research and the previous first year research project. While acknowledging that the proposed approach is based on a relatively limited knowledge and open to criticism, I perceive that this approach addresses a range of important aspects that future AI interventions should be informed by. Furthermore, the main objective of proposing this approach is to shed light on the accompanying activities that could enhance the success of future AI interventions.

Since AI can be used in a variety of situations for various purposes, the proposed approach principally addresses interventions that show similar characteristics (number of summit participants, type of organization and way of operating, etc.) to the three evaluated interventions in this research.

1) Introduction and awareness of AI: In Kotter's research on why transformation efforts fail, one of his arguments is that "major change is impossible unless the head of the organization is an active supporter" (2007, p. 98). This statement might be 
relevant for AI interventions in particular, since AI represents, as Watkins \& Mohr (2001) claim, a new way of seeing and being. Consequently, emphasis needs to be put on this first activity in which top-management learns and buys into this new idea. They need to understand the benefits of using AI, recognize its possibilities and see the potential impact AI can have on the organization. Moreover, top-management needs to understand that $\mathrm{AI}$ is not an event called the summit, it represents an idea on how this organization will do business in the future. The senior managers need to realize that the organization will not be the same after the summit. Knowing what AI is and what it can do, the next step involves becoming clear about the purpose of using AI.

2) Ensuring clarity on why using AI and for what: AI can be used for a variety of objectives and purposes. Whitney \& Cooperrider (1998, p. 21) state, "no matter what the purpose for the summit is, it must be clearly articulated and brought to life during the summit", i.e. the reason for doing an AI intervention must be clear. The interviewees within Org2 in particular claimed that the organization was not explicit about the purpose of doing an intervention. SM1 (Org2) stated, "as an organization we were not sure why we are doing the summit." To tackle this issue, the discussion chapter put forward the idea to categorize the goals and objectives of doing an AI initiative (short-term, long-term, intended goals \& outcomes etc). This activity is intended to support management in creating awareness of all the goals and objectives they want to achieve using an AI intervention. In addition, a categorization might uncover unaligned or even contradict goals and objectives.

3) Identify potential obstacles: Further to knowing what AI is and for what an organization wants to use it for, the organization should also develop a 'list' of possible internal and external factors and obstacles that could interfere with the AI intervention. Bushe (2007) argues that the 'positive nature' of AI alone is unlikely to overcome 'all' the obstacles it might face and consequently wisdom from traditional organizational development (OD) needs to be applied competently. That is, AI is not a 'Wunderwaffe', it is still vulnerable to all the organizational change variables. In creating awareness around issues, obstacles, etc. that could interfere with an AI intervention, an organization gains a position where it can identify these more easily and, if necessary, develop approaches and strategies to counteract them. 
4) Consider the timing of the summit: Choosing 'the right time' to start an AI intervention, seems to be another critical aspect. The question an organization should ask at this stage is: Would the intervention likely be more successful if it was postponed by a month, few months or a year? The significance of the timing aspect should not be underestimated as all of the three cases reviewed show. If certain critical issues and potential obstacles, as identified previously, may dissipate over time, it might be wise simply to wait.

5) Preparation of the actual summit: Depending on the size of an intervention, a significant period of time is needed for the various preparation tasks and activities leading up to the summit. In the three cases presented, it took the organizations between six months to a year to get 'ready' for the summit. Thus, the amount of work should not be underestimated, involving tasks like training of the summit committee in the AI methodology, selection of summit participants, preparation and distribution of information packages for participants, organizing and preparing a venue for the event, etc. As presented in the discussion chapter, the literature on AI does not provide a specifically outlined approach on how to conduct an intervention. Consequently, the intervention itself needs to be customized for an organization and the summit planned with activities that align to AI as well as the organization. However, all three cases indicated that substantial planning preceded a successful summit.

6) Preparation for the time after the summit: The research undertaken here suggests that this might represent one of the most important tasks an organization has to do. An organization needs to be clear about what is going to happen after the summit in order to achieve whatever the organization decides to pursue. Org3 is a good example to illustrate the importance of this point. This organization was not prepared in many ways for the time after the summit, which resulted in disappointment and frustration among some summit participants. Org1, on the other hand, had a clear and wellarticulated concept in place that was immediately put into action after the summit. These examples clearly show that organizations should prepare themselves for the time afterwards and envisage how AI will be used to impact the organization following. 
7) Let the summit do what it does best: After extensive preparation and consideration of the six recommended activities presented above, the organization should be ready for the AI summit. The interviewees across the three cases confirm the literature describing the AI summit as an event resulting in pride, hope, enthusiasm, commitment etc. Building on this feedback from the interviewees, it is suggested to simply let the summit 'do what it does best'. The 4-D cycle (discover, dream, design and destiny) (Cooperrider et al., 2008) appears to be highly effective for engaging participants during the summit.

8) Support employees/summit participants in going back to work: Drawing on the three cases reviewed, the way an organization handles the period right after the summit is likely to have an even bigger impact on the success of an intervention than the summit itself. Within the first few weeks after the summit, the organization has to perform at least two critical tasks.

Firstly, the organization needs to support employees in going through the 'aftershock' in coming back to work. As addressed in the discussion chapter, each summit created expectations and was an amazing experience, while back in the offices it is 'business as usual' with the colleagues that have not have attended the summit. Thus, organizations need to be aware of this issue and address is accordingly. As part of the last phase of the summit, it might be beneficial to 'cool down' the participants, be upfront and clearly state what they can expect from this in the future. Even through it may somewhat contradict with AI's philosophy, participants should leave the summit with a sense of reality and be back on earth after being on 'cloud nine' because the next working day will be reality again.

The second task represents the transference of the 'atmosphere', that was created during the summit, back into the organization and bringing the employees who did not participate in the summit on board. This will not be an easy task to accomplish, especially considering that in the interventions of Org1 and Org2 only $10 \%$ of the total workforce was involved in the summit. Thus, the 'thinking ahead' recommended in task 6, which was developed for the time after the summit, needs to support this task. 
In reviewing these two activities, it appears that they almost contradict each other. On the one hand, summit participants need to 'cool down' and be realistic about their expectations. On the other hand, it is argued as beneficial to transfer the enthusiasm, hype and atmosphere from the summit into the organization. While they may contradict, they are both necessary to retain some of the summit's positive energy and help it to bring change across the organization. Thus, it can be argued that organizations that manage to simultaneously do both things well are more likely to succeed.

9) Follow-up on what was developed for the time after the summit: The approach that was developed for the time after the summit (in task 6) needs to be put in place in order to support AI's integration into the organization. No matter how this approach looks like in detail, its focus and objective should be on engaging 'all' employees by using AI on an ongoing basis and not be limited for the achievement of only specific outcomes. Org1 represents a good example on how the concept of AI could be 'delivered' to an organization. In persistently applying an AI inspired approach over an extended period and constantly reinforcing it, an organization becomes more comfortable in applying AI. In the end it may become just a part of how an organization operates.

Summary: As stated earlier, the objective of proposing these nine tasks that AI interventions should be aware and address accordingly is to shed light on critical aspects in order to enhance the success of future interventions. Still, it is important to consider that this approach proposes just a list of some key aspects future AI interventions should be aware of and not a complete recipe on how to do an intervention. There are additional aspects missing from this list that also need to be handled appropriately. For example the decisions on how many people and whom to invite to a summit were not discussed. However, recognizing this limitation of this proposed approach, future AI interventions should still benefit from acknowledging the nine proposed tasks and discussing their implications.

\subsection{Reflection and implications for further research}

In this research, the data collected in the interviews were presented in making extensive use of quotes. The researcher perceives this approach for presenting data as 
very useful because it provides the reader rich and detailed 'stories', highlighting complexity and aspects that may have impacted the intervention. On the basis of these 'stories', researchers, readers, evaluators, etc. should be able to draw their own conclusions and make judgments about how various aspects affected the success of each intervention. The implications from this research for further studies using a similar approach are threefold.

Reflecting back on the data the interviewees provided, the summits were clearly events that created enthusiasm, excitement and commitment among the participants. In two cases (Org2 \& Org3) these positive developments faded away as time passed by. Thus, the timing of conducting an evaluation is likely to influence the findings i.e. an evaluation that is performed shortly after the summit (e.g. within six months) runs the risk of being deceived by 'positive summit leftovers.' Future research can address this aspect by choosing a longitudinal approach. This means that future evaluationstudies should either collect data at different points in time (e.g. before the summit, right after the summit and two years afterwards) or, as performed in this research, conduct data collection long enough after the summit (likely two years or more) and let the interviewees describe how the organization changed over those years. Two years are considered a suitable point in time to conduct an evaluation, due to the interviewees being able to reflect on the time before the intervention, but also make conclusions regarding the 'long-term' impact of AI.

Due to the purposeful selection of the interviewees, the interviewees could be categorized into three groups per organization, providing the reader several perspectives to look at the interventions. While the interviewees described the summit itself similarly, the time afterwards (particularly in Org2 \& Org3) was experienced differently across the three groups. Including various stakeholder groups in the evaluation process are likely to enhance the quality of evaluation and should make findings more robust. As shown in this research, depending on the perspective the evaluators take, an intervention might be reviewed differently. Therefore, by considering several perspectives in the evaluation process, a more objective and representative evaluation result can be achieved. All the interviewees involved in this research were also part of the AI summits. Future research on evaluating AI interventions should consider including the non-participants' voice, since this 
represents another possible way to evaluate AI's integration into organizational processes.

The last recommendation for future evaluation studies addresses the evaluator themself. It appears to be beneficial deploying an outside person as evaluator. Even though an outside evaluator potentially has less information about the organization, and the process of collecting data is likely to take longer, an internal evaluator runs the risk of already a having predetermined evaluation result in mind. Consequently, the evaluation process by an internal evaluator might be destined to confirm predetermined assumptions, limiting the possibility for new and challenging findings. Future evaluation studies therefore should consider the use of outsiders as evaluators whose interests are unrelated to the evaluation outcomes.

\subsection{Concluding words}

In this research three AI interventions were evaluated. The findings show that the AIsummit's strong focus on positives, strengths, capabilities etc. fostered pride, hope, excitement and enthusiasm among the participants. Org1 successfully managed to transform and integrate these 'outcomes' of the summit into their organization's processes. This organization put weekly meetings in place (started instantly after the summit) and engaged all employees with respect to relevant business topics by applying the AI philosophy. Drawing on AI's philosophy of focusing on what works (instead of what does not), future AI interventions are well advised to put similar approaches in place that follow-up the summit in order to integrate the AI philosophy into their processes and ways of operating. 


\section{Bibliography}

Abama, T. A., \& Stake, R. E. (2001). Stake's responsive evaluation: Core ideas and evolution. New directions for evaluation, 9, 7-21.

Barney, J. B. (2002). Gaining and sustaining competitive advantage (2nd ed.). Upper Saddle River: Pearson Education, Inc.

Barry, D., \& Elmes, M. (1997). Strategy retold: Toward a narrative view of strategic discourse. Academy of Management Review, 22(2), 429-452.

Berrisford, S. (2005). Using Appreciative Inquiry to drive change at the BBC. Strategic Communication Management, 9(3), 22-25.

Buscell, P. (2005). The power of positive deviance. The Organization: A Practicing Manager's Quaterly, 8(1), 38-42.

Bushe, G. R. (2007). Appreciative inquiry is not (just) about the positive. Organization Development Practitioner, 39(4), 30-35.

Bushe, G. R., \& Kassam, A. F. (2005). When is Appreciative Inquiry transformational? A meta-case analysis. The Journal of Applied Behavioral Science, 41(2), 161-181.

Case Western Reserve University (2010). Appreciative Inquiry Commons. Retrieved March 10, 2010: http://appreciativeinquiry.case.edu/community/people.cfm

Collins, J. C., \& Porras, J. I. (1994). Built to last: Successful habits of visionary companies. New York: HarperCollins Publishers, Inc.

Cooperrider, D. L. (1986). Appreciative inquiry: Toward a methodology for understanding and enhancing organizational innovation. Case Western Reserve University, Cleveland, $\mathrm{OH}$. 
Cooperrider, D. L., \& Srivastva, S. (1987). Appreciative Inquiry in organizational life. Research in Organizational Change and Development, 1, 129-169.

Cooperrider, D. L., \& Whitney, D. (1999). Aprreciative Inquiry (1st ed.). San Francisco: Berret-Koehler Communications, Inc.

Cooperrider, D. L., \& Whitney, D. (2005). Appreciative Inquiry - A positive revolution in change (1st ed.). San Francisco: Berret-Koehler Publishers, Inc.

Cooperrider, D. L., Whitney, D., \& Stavros, J. M. (2008). Appreciative Inquiry handbook: For leaders of change (2nd ed.). San Francisco: Berret-Koehler Publishers, Inc.

Cunningham, G., \& Mathie, A. (2002). Asset-based community development: An overview. Antigonish, Canada: Coady International Institute.

Dinesen, M. S. (2009). Systemic appreciative evaluation: Developing quality instead of just measuring it. AI Practitioner, 11(3), 49-56.

Dunlap, C. A. (2008). Effective evaluation through appreciative inquiry. Performance Improvement, 47(2), 23-29.

Easterby-Smith, M., Thorpe, R., \& Jackson, P. R. (2008). Management Research (3rd ed.). London: Sage Publications, Ltd.

Egan, T. M., \& Lancaster, C. M. (2005). Comparing Appreciative Inquiry to action research: OD practitioner perspectives. Organization Development Journal, 23(2), 29-49.

Eriksson, P., \& Kovalainen, A. (2008). Qualitative Methods in Business Research (1st ed.). London: Sage Publications, Ltd.

Faure, M. (2006). Problem solving was never this easy: Transformational change through Appreciative Inquiry. Performance Improvement, 45(9), 22-32. 
Grant, S., \& Humphries, M. (2006). Critical evaluation of Appreciative Inquiry. Action Research, 4(4), 401-418.

Greene, J. C., \& Caracelli, V. J. (1997). Advances in mixed-method evaluation: The challenges and benefits of integrating diverse paradigms: New Directions for Evaluation. San Francisco: Jossey-Bass Publishers.

Kotter, J. P. (2007). Leading change: Why transformation efforts fail. Harvard Business Review, 85(1), 96-103.

Ludema, J. D., Whitney, D., Mohr, B. J., \& Griffin, T. J. (2003). The Appreciative Inquiry Summit: A practitioner's guide for leading large-group change (1st ed.). San Francisco: Berrett-Koehler Publishers, Inc.

Mayan, M. J. (2001). An introduction to qualitative methods: A training module for students and professionals (1st ed.). Alberta: International Institute for Qualitative Methodology.

Mellish, E. E. (2007). Appreciative Inquiry at work - Lessons from case study research for AI practitioner and their clients. AI Practitioner, 30-35.

Mendator, D. (1999). Measuring the unmeasurable. In P. Senge, A. Kleiner, C. Roberts, R. Ross, G. Roth \& B. Smith (Eds.), The dance of change (1st ed., pp. 311-313). London: Nicholas Brealey Publishing.

Messerschmidt, D. (2005). A qualitative review of the impatcs of Appreciative Inquiry. Used by UNICEF/Nepal, 2000-2004. Kathmandu: Women's Health Project, UNICEF/Nepal.

Messerschmidt, D. (2008). Evaluating Appreciative Inquiry as an organizational transformation tool: An assessment from Nepal. Human Organization, 67(4), 454-468. 
Miller, M. G., Fitzgerald, S. P., Murell, K. L., Preston, J., \& Ambekar, R. (2005). Appreciative Inquiry in building a transcultural strategic alliance. The Journal of Applied Behavioral Science, 41(1), 91-110.

Ncube, L. B., \& Wasburn, M. H. (2008). Strategic analysis: Approaching continuous improvement proactively. Review of Business, 29(1), 15-25.

O'Leary, Z. (2004). The essential guide to doing research (1st ed.). London: Sage Publications, Ltd.

Preskill, H., \& Catsambas, T. T. (2006). Reframing evaluation through Appreciative Inquiry. Thousand Oaks: Sage Publications, Inc.

Reed, J. (2007). Appreciative Inquiry: Research for change. California: Sage Publications, Inc.

Rogers, P. J., \& Fraser, D. (2003). Appreciating Appreciative Inquiry. New Directions for Evaluation, 2003(100), 75-83.

Roth, G. (1999). Cracking the "Black Box" of a learing initiative assessment. In P. Senge, A. Kleiner, C. Roberts, R. Ross, G. Roth \& B. Smith (Eds.), The dance of change (1st ed., pp. 303-311). London: Nicholas Brealey Publishing.

Salopek, J. J. (2006). Appreciative Inquiry at 20: Questioning David Cooperrider. $T+$ $D, 60(8), 21-22$.

Senge, P., Kleiner, A., Roberts, C., Ross, R., Roth, G., \& Smith, B. (1999). The dance of change (1st ed.). London: Nicholas Brealey Publishing.

Shaw, G., Brown, R., \& Bromiley, P. (1998). Strategic stories: How 3M is rewriting business planning. Harvard Business Review, 76(3), 41-50.

van der Haar, D., \& Hosking, D. M. (2004). Evaluating appreciative inquiry: A relational constructionist perspective. Human Relations, 57(8), 1017-1036. 
Watkins, J., \& Mohr, B. (2001). Appreciative Inquiry: Change at the speed of imagination. San Francisco: John Wiley \& Sons, Inc.

Whitney, D. (1998). Let's change the subject and change our organization: An Appreciative Inquiry approach to organization change. Career Development International, 3(7), 314-319.

Whitney, D., \& Cooperrider, D. L. (1998). The Appreciative Inquiry Summit: Overview and applications. Employment Relations Today, 25(2), 17-28.

Whitney, D., Cooperrider, D. L., Trosten-Bloom, A., \& Kaplin, B. S. (2005). Encyclopedia of positve Questions: Using Appreciative Inquiry to bring out the best in your organization (Vol. 1). Brunswick, $\mathrm{OH}$ : Crown Custom Publishing.

Whitney, D., \& Trosten-Bloom, A. (2003). The power of Appreciative Inqury (1st ed.). San Francisco: Berret-Koehler Publishers, Inc. 


\section{Appendices}

Appendix A:

Information Sheet

\section{VICTORIA UNIVERSITY OF WELLINGTON \\ Te Whare Wānanga o te Ûpoko o te Ika a Māui

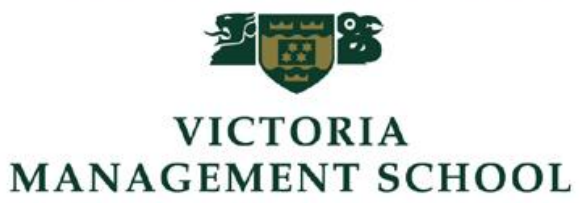

Te Kura Whakahaere

\section{Information Sheet}

\section{Research project: Evaluation of Appreciative Inquiry interventions}

I am a student at Victoria University of Wellington doing a Masters degree in Management Studies (MMS). This research is being conducted as part of the requirements for the completion of my degree and written up as a Masters thesis.

In this research, I aim to examine the impact of the recently completed Appreciative Inquiry event on your organization. The objective is to find evidence of whether changes occurred and elaborate whether this process fulfilled its expectations. These interviews can contribute in our understanding of the dynamics behind Appreciative Inquiry interventions and add valuable insights for their improvement.

\section{How will you be affected by being one of my interviewees}

- Participation is entirely voluntary. You are not obliged to participate.

- If you agree, the interview will be 30-60 minutes long and scheduled at a time that suits you.

- You have the right to withdraw yourself or any information you have provided from this project without having to supply a reason for doing so. In which case, information obtained will be immediately destroyed.

- The researcher will not discuss your participation or your interview with any other employees of your firm.

- I would like to record the interview, but this would only be done with your consent. The digital recording will be transcribed by me and only my supervisor has additional access to the recording and the transcript.

- I will be asking you about your views, opinions and experiences in using Appreciative Inquiry. All information gathered in these interviews will be treated confidentially - your name will not be used. The interview will be reported in an aggregated non-attributable form. E-Mail me, if you would like an electronic copy of the final results of this study.

- Ethical approval from Victoria University of Wellington has been given for the proposed research.

- All participants will sign a Research Agreement where they can state how they would like the data collected from them to be handled.

\section{Contact Information}

Thank you for you time and help to make this study possible. If you have any queries please do not hesitate to contact me or my supervisor, Urs Daellenbach, using the contact details below.

Researcher: Martin Stellnberger

Masters of Management Studies

Victoria Management School

Victoria University of Wellington

Phone: $0210 \operatorname{xxxxxx}$

Email: martin.stellnberger@vuw.ac.nz
Supervisor: Associate Prof. Urs Daellenbach

Victoria Management School

Victoria University of Wellington

Phone: 04 xxx xxxx

Email: urs.daellenbach@vuw.ac.nz 
Appendix B:

Research Agreement

\section{VICTORIA UNIVERSITY OF WELLINGTON \\ Te Whare Wānanga o te Ûpoko o te Ika a Māui

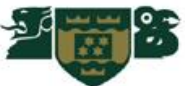 \\ VICTORIA \\ MANAGEMENT SCHOOL}

Te Kura Whakahaere

\section{Research Agreement}

\section{Research Title:}

Evaluation of Appreciative Inquiry Interventions

\section{Researcher:}

Martin Stellnberger, Master of Management Studies, Victoria Management School, Victoria University of Wellington.

\section{Purpose of agreement:}

This agreement is to ensure that you are sufficiently informed about the purpose of the research, and your right to know how data will be collected, analyzed and written up.

\section{Consent to participation}

I have been given and have understood an explanation of this research project.

I have had an opportunity to ask any questions and had them answered to my satisfaction.

I understand the data collected will remain confidential and will be reported in an aggregated, non-attributable form.

I understand that I may decline to answer any question asked in this interview.

$\square$ I understand that I may withdraw myself or any information I have provided from this project (before data analysis is completed (likely end of July 2010)), without having to supply a reason for doing so. In which case, information obtained will be immediately destroyed.

$\square$ I understand that the information obtained will be stored in a locked cabinet or passwordprotected file. All recordings will be wiped after they have been transcribed.

$\square$ A copy of the research paper or thesis will be deposited in the Victoria University of Wellington Library. Findings may be presented at conferences or published in academic or professional journals at a later date. This will mean that the anonymized transcripts may be kept securely for up to 2 years. Any further use will require my written consent.

I agree to participate in this study.

I agree to the interview being recorded.

\section{Participant}

Name:

Organization:

Date:

Signature:

\section{Researcher}

Martin Stellnberger

Masters of Management Studies

Victoria University of Wellington

Signature: 
Appendix C:

Interview Schedule

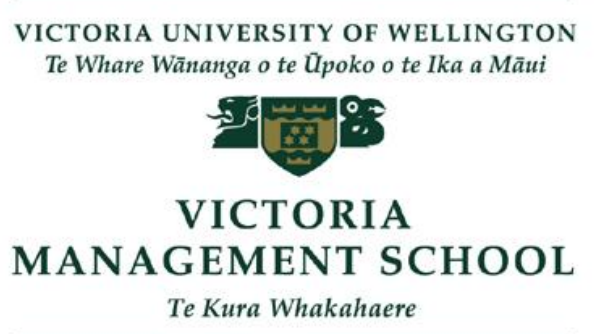

Evaluation of Appreciative Inquiry interventions

Interview Schedule

\section{Section 1: Intro \& Administration}

- Information sheet

- Research agreement

\section{Section 2: Participant background}

Can you briefly describe your background in working for this organization?

- For how long have you worked for this organization / in this position?

- Can you tell me about your job? What are your tasks and responsibilities?

\section{Section 3: The intervention}

I would like to understand your organization's ways of doing things a little bit more.

\begin{tabular}{|l|l|c|c|}
\hline & Before & $\begin{array}{c}\text { After the } \\
\text { intervention } \\
(\sim 1 \text { month) }\end{array}$ & $\begin{array}{c}\text { x months after the } \\
\text { intervention } \\
\text { (Now) }\end{array}$ \\
\hline Process & $\bullet$ & $\bullet$ & $\bullet$ \\
\hline Communication & $\bullet$ & $\bullet$ & $\bullet$ \\
\hline Culture & $\bullet$ & $\bullet$ & $\bullet$ \\
\hline
\end{tabular}

- Process: How were things done [before/directly after/X months after (now)] the intervention?

- Communication: How was information handled and shared [before/directly after/X months after (now)] the intervention?

- Culture: How would you characterize your organization's norms and values [before/directly after/X months after (now)] the intervention?

- What do you think were the expectations and goals of doing AI?

- What do you think was the effect of AI on the organization? Can you please give examples.

- What do you think was the effect of AI on your every day working life? What do you think are the benefits of doing this?

Are there any drawbacks?

\section{Section 4: Conclusion and final though}

- If you could write one paragraph on the topic "Appreciative Inquiry in my organization" what would this paragraph likely include? 